\title{
eJRIEPS
}

Ejournal de la recherche sur l'intervention en éducation physique et sport

$19 \mid 2010$

Varia

\section{Les recherches ARIS : contextes d'intervention étudiés et thématiques de recherche}

Mathilde Musard, Jean-François Robin et Marie-Paule Poggi

\section{(2) OpenEdition}

\section{Journals}

Édition électronique

URL : http://journals.openedition.org/ejrieps/5462

DOI : 10.4000/ejrieps.5462

ISSN : 2105-0821

Éditeur

ELLIADD

\section{Référence électronique}

Mathilde Musard, Jean-François Robin et Marie-Paule Poggi, «Les recherches ARIS : contextes d'intervention étudiés et thématiques de recherche », eJRIEPS [En ligne], 19 | 2010, mis en ligne le 01 janvier 2010, consulté le 08 janvier 2021. URL : http://journals.openedition.org/ejrieps/5462 ; DOI : https://doi.org/10.4000/ejrieps.5462

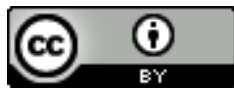

La revue eJRIEPS est mise à disposition selon les termes de la Creative Commons Attribution 4.0 International License. 
Les recherches ARIS :

contextes d'intervention étudiés et thématiques de recherche

Mathilde Musard, Jean-François Robin* \& Marie-Paule Poggi

GRIAPS/LaSelDi (EA 2281) Université de Franche-Comté

* Université Paris Est Créteil, Département STAPS, GéDIAPS

\section{Résumé}

Cette étude s'inscrit dans la première mission de I'ORIS (Observatoire des Recherches sur l'Intervention en Sport) à savoir "mutualiser, caractériser et synthétiser les connaissances produites". Elle vise à construire un état des lieux des communications produites au cours des cinq premiers congrès de l'ARIS, de 2000 à 2008. Après avoir caractérisé dans le premier article de ce numéro (Musard, Poggi \& Wallian, 2010) le public concerné par les congrès de l'ARIS, les différents types de communications produites et les recherches (finalités, méthodologies, champs scientifiques de référence), nous nous centrons désormais sur les recherches pour identifier les secteurs d'intervention étudiés et présenter les divers objets d'étude traités dans les recherches sur l'intervention en sport.

Dans la continuité du premier article publié dans ce numéro (Musard, Poggi \& Wallian, 2010), nous poursuivons le panorama des biennales de l'ARIS (Association pour la Recherche sur l'Intervention en Sport), de 2000 à 2008, en se centrant sur les recherches $(\mathrm{n}=545)$. Il s'agit de présenter plus précisément les secteurs d'intervention étudiés et les divers objets d'étude traités dans les recherches sur l'intervention en sport. Cette étude offre donc au lecteur une vision d'ensemble des recherches; de nombreuses communications des congrès de l'ARIS, orales ou affichées, sont citées à titre d'exemple. Les lecteurs souhaitant approfondir ces recherches pourront se référer aux actes des colloques (Aubert, Durand \& Refuggi, 2001 ; Loquet \& Léziart, 2004 ; Bui-Xuan, Bouthier \& Carlier, 2006 ; Wallian, Poggi \& Musard, 2008 ; Amade-Escot \& al, sous presse) et à certains numéros spéciaux de la revue Ejrieps (eJ 18 ; eJ 19).

\section{Contextes d'intervention étudiés}

La figure 1 fait apparaître trois secteurs d'intervention principalement étudiés lors des biennales de l'ARIS : 
- l'éducation physique et sportive (EPS), qui concerne plus de la moitié des recherches $(55,6 \%)$;

- la formation (des enseignants, entraîneurs, formateurs...) $(23,3 \%)$;

- l'entraînement (18,7\%).

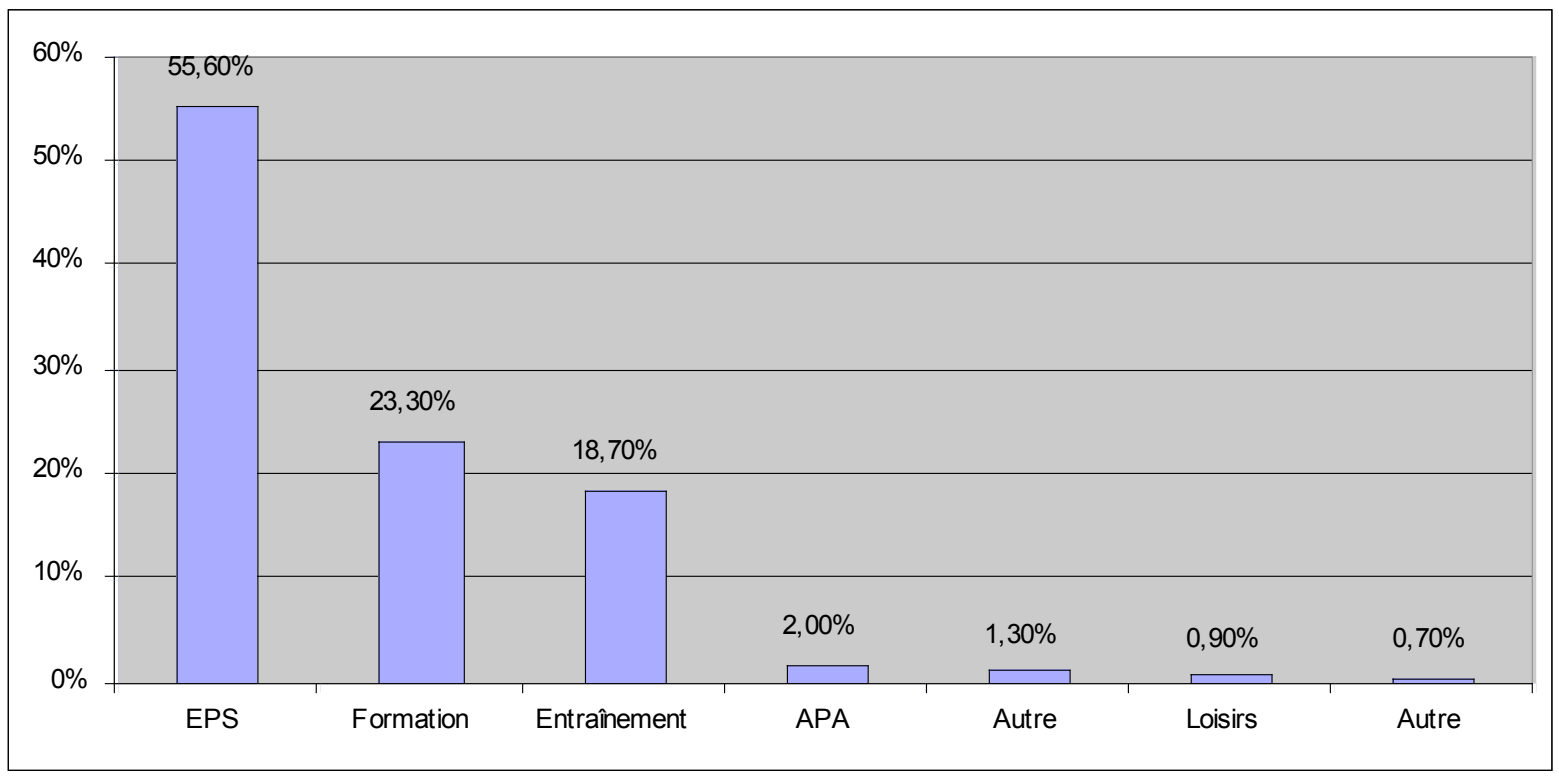

Figure 1. Contextes d'intervention étudiés dans les recherches ARIS

Ces résultats sont stables de 2000 à 2008. Ainsi, les chercheurs intéressés par l'enseignement et la formation participent largement aux congrès de l'ARIS, lieu de rassemblement et d'échanges. Ces données peuvent néanmoins questionner les membres de l'ARIS, dans la mesure où cette association cherche à regrouper, voire à comparer les travaux liés à l'activité motrice dans les différents contextes d'intervention en sport. Or, certains domaines tels que les activités physiques adaptées (APA), les loisirs, sont moins investigués que l'EPS, la formation ou l'entraînement. II serait souhaitable que ces secteurs d'intervention soient davantage représentés lors des colloques à venir. En effet, ce déséquilibre quantitatif rend difficile une comparaison raisonnable.

Précisons que les recherches sur les APA $(n=11)$ s'intéressent aussi bien à l'activité des intervenants (par exemple avec des adolescents autistes en danse (Roncin \& Loquet, 2006), aux populations en difficultés d'adaptation sociale (Mikulovic, Bui-Xuan \& Theunynck, 2005), aux élèves en enseignement spécialisé (Dupont, 2008)) qu'à celle des pratiquants (les adultes handicapés mentaux (Redon, 2006) et les personnes âgées (Santiago \& Ribeiro, 2006)). Les recherches sur les loisirs sont plutôt centrées sur le rôle social du sport (Beltramini, Delfosse, Piéron, \& Cloes, 2000 ; Carnel, Masschelein \& 
Boutoille, 2008 ; Vandermeulen \& Cloes, 2008). D'autre part, certaines recherches étudient le domaine pré-scolaire, notamment les interactions enfants-parents lors de l'activité aquatique d'éveil (Roesslé \& Loquet, 2006). Enfin, dans la catégorie « autre », la plupart des recherches propose une analyse comparative entre l'EPS et différentes disciplines scolaires (Lemonie, Robin, Le Gall \& David, 2008 ; Roustan, 2005).

Dans les parties suivantes, nous présentons les thématiques de recherche qui émergent de l'analyse de contenu des programmes scientifiques dans les trois principaux contextes étudiés au sein de l'ARIS : I'EPS, la formation et l'entraînement.

\section{L'EPS}

Instruction et gestion

de la classe

\section{La formation}

Compétences, contenus et dispositifs d'apprentissage

Perceptions, expériences des

élèves

Genre, mixité

Stratégies d'élèves, interactions

langagières et apprentissage

Savoirs professionnels

Planification

Interactions enseignant -

élèves

Evaluation

et

Développement de l'activité

Professionnelle

Perceptions et stratégies

des formés

Travail du formateur

Dispositif de formation

innovant

Interactions formateur-formé

\section{L'entraînement}

Perceptions et stratégies

des sportifs

Intervention de l'entraîneur

Optimisation de

l'entraînement

Analyse du jeu

Interactions entraîneur-

entraîné

Activité de l'arbitre

Tableau I. Thématiques de recherches présentées dans les contextes de l'EPS, de la formation et de l'entraînement 


\section{Les recherches sur I'EPS}

2. 1. Les thématiques de recherches

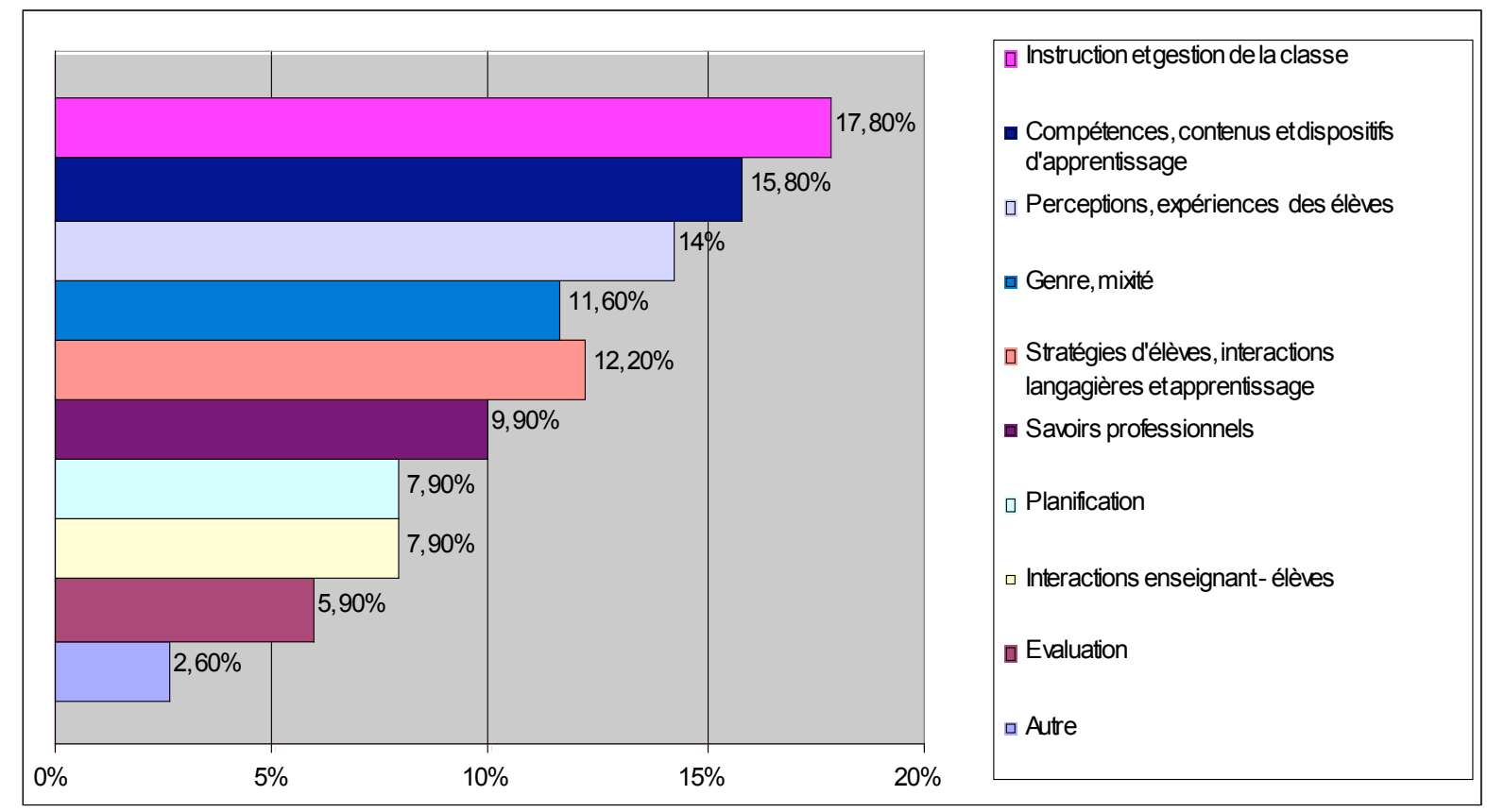

Figure 2. Thématiques des recherches sur l'EPS

La figure 2 met en évidence la variété des thématiques étudiées dans les recherches en EPS, avec une dizaine de catégories qui émerge de l'analyse de contenu des résumés. L'activité de l'enseignant reste plus largement étudiée. En effet, les catégories relatives à l'enseignant (« instruction et gestion de la classe » : 17,8\%; « savoirs professionnels » : 9,9\% ; « planification » : 7,9\% ; « évaluation » : 5,9\%) comptent environ pour 40\% des recherches. Mais l'activité des élèves n'est pas pour autant délaissée, puisqu'un quart des recherches s'y consacre («perceptions, expériences des élèves »: 14\% ; "stratégies d'élèves, interactions langagières et apprentissage » : 12,2\%). La réflexion curriculaire sur les compétences, contenus et dispositifs d'apprentissage est assez peu étudiée (15,8\% des recherches). Nous pouvons aussi remarquer que l'activité de l'enseignant et celle des élèves ne sont pas systématiquement étudiées de façon isolée. En effet, $8 \%$ des recherches s'intéressent aux interactions enseignant - élèves. Enfin, 11,6\% des recherches s'orientent vers le thème du genre et de la mixité. Cette répartition des thématiques de recherches sur l'EPS reste stable de 2000 à 2008. La diversité des thèmes étudiés est d'ailleurs un signe de richesse, car la recherche francophone sur l'intervention en sport progresse parallèlement sur de nombreux sujets. Aux EU, Silverman \& Manson (2003) soulignent qu'après s'être largement focalisées sur l'efficacité de l'enseignant, les recherches en EPS se sont aussi orientées vers l'étude de l'activité de 
l'élève, notamment sur la cognition et les perceptions des élèves, et plus seulement sur l'apprentissage moteur.

D'autre part, les recherches portent essentiellement sur l'EPS dans le second degré : le premier degré est assez peu investigué (16,2 \% des recherches), tout comme le sport scolaire $(2,3 \%)$. Nous notons néanmoins une augmentation des recherches en école primaire de $2006(14,7 \%)$ à $2008(21,1 \%)$, et pouvons souhaiter que cette tendance perdure, afin que les connaissances produites sur le premier degré s'accroissent. Cette tendance s'accentue tout particulièrement en France. Dans les autres pays, les recherches sur l'école primaire sont plus importantes, notamment au Canada (63, 9\% des recherches ARIS) et aux Etats Unis (plus d'un tiers des recherches en EPS ; Silverman \& Skonie, 1997 ; Silverman \& Manson, 2003), où l'EPS, particulièrement bien développée dans le premier degré, n'est pas obligatoire dans le secondaire.

Chacune des thématiques de recherches sur l'EPS (figure 2) est illustrée ci-dessous à travers de nombreux exemples de recherches présentées lors des congrès ARIS.

\section{Instruction et gestion de la classe}

Une première catégorie de recherches se focalise sur la gestion de la classe. Les auteurs identifient les facteurs à l'origine des incidents disciplinaires en cours d'EPS (Dervaux, Carlier et Gérard, 2005 ; Masson, Carnel et Garnier, 2005) et montrent par exemple l'importance commune accordée au plaisir d'enseigner et d'apprendre par l'enseignant et par les élèves (Haye, 2005 ; Carlier, Faber \& Petesch, 2008). Selon Flavier, Bertone et Méard (2000), on retrouve une organisation typique des conflits professeur - élève(s) en collège, comme la réaction rapide et discutable de l'enseignant et le sentiment d'injustice ressenti par les élèves. Plus particulièrement, en milieu difficile, l'intervention se caractérise par un contrôle plus important des élèves, des interactions verbales très nombreuses laissant peu de place à l'autonomie et l'éclatement des contenus (Debars \& Amade-Escot, 2005). Les activités contradictoires des élèves et de l'enseignant reflètent une structuration typique (actions ostensives ou actions masquées), mais elles se coordonnent pour produire une activité collective viable compatible avec les exigences de travail scolaire de l'enseignant (Vors, Gal-Petitfaux \& Ciceron, 2008). II est donc possible de maintenir un enseignement de l'EPS démocratique et égalitaire (Martel, Gagnon \& Demers, 2002), ne lâchant pas les apprentissages des élèves (Falga \& Monnier, 2008).

Une seconde catégorie d'études se centre sur les gestes professionnels de l'enseignant, (genre et style) qui renvoient à certaines fonctions et techniques didactiques (Garnier, 2005). S’ils présentent une dimension générique, ils se caractérisent aussi par une dimension spécifique et geste diffèrent par exemple selon les disciplines enseignées 
(Robin \& David, 2006 ; Roustan, 2005), selon l'activité sportive enseignée (Legrand \& Refuggi, 2006), selon l'expertise enseignante (Kammoun \& Amade-Escot, 2005 ; El Andoulsi \& Bedhioufi, 2008 ; Durny \& al, 2000), en fonction des épisodes de la séance (Trottin \& Bazogue, 2002) ou encore du contexte d'intervention (Frédéric \& Cloes, 2008). Une troisième catégorie de recherches se focalise sur la communication non verbale (Mahut, Mahut \& Gréhaigne, 2000 ; Breton, Mahut \& Gréhaigne, 2005) ou verbale de l'enseignant. Les différences langagières entre enseignants novices et enseignants expérimentés ne sont pas sans effet sur les apprentissages (Touboul, Carnus \& Terrisse, 2008). Ainsi, les chercheurs montrent comment les enseignants structurent leur discours pour inciter les élèves à réaliser efficacement les habiletés motrices (Gal-Petitfaux, 2006 ; Vercruysse, Paquay \& Carlier, 2008) ou utilisent des stratégies d'alternance codique aux Antilles Françaises (français/créole) en vue de favoriser les apprentissages après d'élèves bilingues (Anciaux et Alin, 2006).

D'autres recherches se focalisent sur les stratégies d'observation des enseignants (Kohler \& Gréhaigne, 2000 ; Dubé \& Delens, 2000 ; Desbiens, 2002) et mettent en évidence différents registres interprétatifs (Voisard, Nachon \& Mahut, 2002 ; Clerc, Wallian, Musard \& Poggi, 2005 ; Outrey, Mahut \& Gréhaigne, 2000). Gouju (2005) analyse plus particulièrement le phénomène de «l'œil du maquignon », une compétence professionnelle jamais indépendante d'arrière-plans.

Enfin, d'autres communications se situent dans cette thématique « gestion et instruction de la classe » et ciblent des moments d'intervention ou des compétences professionnelles particulières. Citons :

- la situation de début de leçon (Lacroix, Gal-Petitfaux \& Cizeron, 2002), les informations collectives données par l'enseignant (Cloes, Bonfond \& Lenzen, 2002) et ses attentes (Trouilloud \& Sarrazin, 2000);

- le processus décisionnel de l'enseignant (Carnus, 2002) marqué par le poids de l'histoire personnelle des acteurs (Loizon, 2005); le travail curriculaire de l'enseignant dans le cadre de la mise en œuvre d'un nouveau programme (Borges \& Rondea, 2008) ; les régulations didactiques (Boudard, 2006) ;

- le processus de dévolution du savoir en basket-ball (Thépaut \& Léziart, 2005) et en équitation (Sicart \& Maurice, 2006) ;

- les transactions et les émotions des enseignants experts (Aymé, Ferrand \& Cogérino, 2005 ; Visioli \& Ria, 2008);

- La nature de la collaboration en classe entre deux enseignants à l'école primaire (Devos-Prieur \& Amans-Passaga, 2008 ; Massey \& Grenier, 2008) 


\section{Compétences, contenus et dispositifs d'apprentissage}

Les chercheurs s'intéressent à diverses compétences transversales (le développement de l'estime de soi (Bergé, 2006), de la responsabilité (Beaudoin, Harnois \& Brunelle, 2008) ou l'éducation à l'environnement pour un développement durable (Ballot, Musard \& Grandclément, 2008)). Mais c'est la problématique de l'éducation à la santé et de son intégration en EPS qui fait l'objet de nombreuses études, notamment au Canada. Celles-ci s'intéressent aux savoirs que les enseignants considèrent comme essentiels et aux stratégies d'enseignement mises en œuvre (Beaudoin, Rivard, Grenier \& Gréhaigne, 2006 ; Borges, Kalinova \& Le Cren, 2006 ; Berchebru \& Poggi, 2006 ; Turcotte et al., 2008 ; Lafont, Rubert \& Vincent-Morin, 2008 ; Desbiens, Spallanzani \& Tourigny, 2008 ; Bizzonni-Previeux, Grenier, Merini, Jourdan \& Otis, 2008). Différentes approches de l'éducation à la santé, axées sur les savoir-faire ou privilégiant les savoirs théoriques sont dégagées par Turcotte, Gaudreau et Otis (2006). Des propositions de contenus interdisciplinaires (Perrin \& Lefèbvre, 2006 ; Cogérino, Perrin \& Motta, 2000) et d'activités de type « défi » (Michaud, Gauthier, Gagnon \& Demers, 2005) sont présentées.

D'autre part, les compétences spécifiques aux APSA (par exemple, la composante artistique en danse, Grandclément \& Musard, 2008) font également l'objet d'études.

La rupture de sens entre pratiques scolaires et pratiques de référence, les décalages observés entre contenus programmés et contenus enseignés amènent les auteurs à se questionner sur les connaissances des enseignants en natation pour Attiklémé \& Refuggi (2002) et en danse pour Coltice (2000). Kirk \& Kirk (2008) analysent alors les effets du modèle «Sport Education » proposé par Siedentop (1994) en Angleterre. Des études d'ingénierie didactique sont aussi menées pour élaborer des contenus qui respectent les finalités éducatives scolaires sans perdre le sens social qui s'attache à la référence (en gymnastique rythmique, Loquet, 2000 ; en danse indienne Kathak, Ranganathan \& Loquet, 2002). Ainsi, des recherches technologiques, visant la transformation des pratiques, sont menées dans différentes APSA. Des outils didactiques au service de l'enseignement des activités gymniques sont proposés, par exemple des tableaux organisateurs de la motricité gymnique permettant de programmer les contenus en niveaux d'habiletés par familles d'actions (Lorrain \& Froissart, 2006), ainsi que des propositions pour valoriser la dimension acrobatique de l'acrosport (Caty \& Musard, 2006). L'efficacité de différents programmes est testée, par exemple: des programmes visant le développement de la coordination chez les élèves (Cousin, Brunelle, Dugas \& 
Spallanzani , 2006 ; Pehoiu, Savu \& Chesnoiu, 2008), un programme de pentathlon en équipe pour inciter les jeunes à la pratique d'activité physique (Gagnon, Nadeau, Martel, \& Normandin , 2006), un programme de sport-études pour favoriser la socialisation des élèves (Frédéric, Louis \& Cloes, 2005), des outils pour aider les enseignants à concevoir et à gérer les cycles d'athlétisme (Gérard, 2002 ; Generolo, Zaragoza, Julian \& Lopez, 2006 ; Pehoiu, 2006 ; Dusson \& Marchal, 2002).

Enfin, d'autres recherches se centrent sur les effets des formes de regroupement des élèves sur l'enseignement et l'apprentissage (Cizeron \& Gal-Petitfaux, 2005 ; Bordes, 2005) ou de différentes méthodes d'enseignement (pédagogie par objectifs et approche par compétences en sports collectifs; aménagement du milieu et débat d'idées, Rezig, Zghibi \& Gréhaigne, 2006 ; Château, Gréhaigne \& Caty, 2005).

\section{Expériences et perceptions des élèves}

Les préoccupations des élèves sont analysées en exploitant le cadre théorique et méthodologique du cours d'action en tennis de table (Guérin, 2006), en badminton (Guillou \& Durny, 2006 ; Huet, Saury \& Rossard, 2006 ; Rossard, Leveau \& Testevuide, 2002) et en hand-ball (Trohel \& Crance, 2008). Guillou \& Durny (2000) observent que les élèves, dans une situation de volley-ball, ne sont pas focalisés par les difficultés qu'avaient identifiées l'enseignant au préalable, car ils se font leur propre représentation de la situation. De même, Kermoal \& Gréhaigne (2006) mettent en évidence que l'élève en cours d'apprentissage en saut en hauteur ne se comporte pas en simple récepteur de coordinations motrices attendues, mais il construit son activité de saut en hauteur dans un espace singulier reposant sur les propriétés qu'il prête à la situation et sur les actions compatibles que ces propriétés induisent. Ces résultats invitent à construire un projet d'enseignement davantage centré sur les intentions et les préoccupations des élèves. En s'appuyant sur la didactique et la clinique de l'activité, Magendie (2008) montre l'intérêt d'analyser l'activité des élèves en prenant en compte ce qu'il est possible ou impossible de faire, et invite les enseignants à faire des hypothèses sur les raisons qui poussent les élèves à agir de la sorte et à circonscrire au mieux les zones de développement du sens et de l'efficience. Wallian, Poggi \& Bonfils (2008) s'orientent vers la sémiotique pour comprendre comment se croisent les subjectivités des joueurs en hand-ball, comment se jouent les rôles en fonction des rapports de place, et finalement comment se construit la communauté de pratiques au fil du jeu.

D'autre part, les perceptions des élèves sont analysées à propos de différents thèmes :

- le degré de concordance (écart entre l'image qu'un élève se fait de lui-même et ce qu'il croit que son éducateur physique pense de lui). Cet indice est révélateur de 
l'influence des comportements d'un éducateur physique (Gagnon, Martel, Michaud, Valois \& Gagné, 2002 ; Martel, Gagnon, Godbout, 2008). Les élèves qui s'estiment sous-évalués par leur enseignant sont frustrés et déclarent réagir par des comportements négatifs. A l'inverse, la conduite de l'éducateur physique fait vivre des émotions positives à d'autres élèves, ce qui les incite à se comporter de façon pertinente au gymnase ;

- les iniquités perçues par les élèves en cours d'EPS (Brunelle \& al., 2000 ; Lentillon \& Cogérino, 2002). Elles sont décrites selon deux grandes familles "d'injustices" : les évènements liés à l'application de sanctions et les évènements relatifs à la gestion de classe et aux activités d'apprentissage ;

- les perceptions des élèves vis-à-vis de la pratique d'une l'activité sportive (Camire \& Trudel, 2008 ; Cloes, Maraite, Zhang, Albert \& Theunissen (2008), des opportunités de pratique (Bothelo-Gomes, Graça, Siva \& Queiros, 2005 ; Zafour \& Hamdi, 2005), ou des obstacles (Longchamp et Cogérino, 2006);

- L'analyse des perceptions des élèves à propos de leurs attentes vis-à-vis de l'EPS. Vigneron (2005) montre qu'une perspective sportive, compétitrice, performante de la discipline va de pair avec un haut niveau de réussite alors qu'une approche plus socio-ludique ou hygiénique obère considérablement les chances de briller au baccalauréat ;

- Les perceptions des élèves à propos de ce qu'il faut faire pour réussir à l'école (Beaudoin, Leblanc \& Lauzon, 2005) ou en EPS (Boudreau, 2005). Il est démontré qu'en EPS l'item " faire des efforts " concerne $90 \%$ des élèves, puis quatre autres énoncés sont choisis (" avoir un bon comportement ", "améliorer sa condition physique ", "collaborer avec les autres" et "avoir sa tenue »), mais peu d'importance est accordée aux apprentissages ;

- Les points de vue des élèves à propos des compétences professionnelles des enseignants (Vigneron, 2005) ou de leur soutien (Lentillon \& Cogérino, 2005). On retient que les élèves privilégient l'aspect relationnel et apprécient la plus grande proximité avec des enseignants jeunes.

\section{Stratégies d'élèves, interactions langagières et apprentissage}

Un nombre conséquent de recherches s'attache à décrire les différentes stratégies des élèves. Ainsi, en course d'orientation, Bonnard et Wallian (2006) questionnent les stratégies interprétatives des élèves et montrent comment la confrontation avec le terrain les amène à reformuler leur projet tactique en cours d'action. De même, en volley-ball, les élèves adaptent leurs plans en fonction des facteurs contextuels au cours de l'action 
(Blocher \& Durny, 2002). La variété des stratégies des élèves est aussi soulignée par Thépaut (2006) au cours de matchs de fin de leçons de basket-ball : si certains élèves jouent sans tenir compte de ce qui a été vu lors des situations d'apprentissage, d'autres souhaitent mettre en œuvre le savoir travaillé, au détriment de la logique du jeu. Ces stratégies d'élèves sont décrites dans diverses APSA : en pentathlon en équipe (Gagnon, Normandin, Nadeau, \& Martel \& Michaud, 2006), en athlétisme (Oudot \& Poggi, 2008), en lutte (Sauvegrain, Carnus, Terrisse \& Loizon, 2005), dans un cycle de jeux d'invasion (Kirk, MacPhail \& Griffin, 2005), en gymnastique (LeBas, 2000) ou en course longue (Lebouvier \& Ouitre, 2002). D'autres chercheurs s'intéressent plus particulièrement aux stratégies d'autorégulation (Kermarrec et Léziart, 2002), et montrent que l'autorégulation de la motivation s'effectue à partir d'une succession de buts personnels que se fixent les élèves, qui peuvent être proches et lointains (Coignus, Famose \& Margnes, 2008). Quant à Chigot, Grison et Riff (2000), ils s'orientent vers une analyse située et distribuée des élèves en Rock'n'Roll, en se centrant sur la spécificité d'un réseau, dans une dynamique de fonctionnement à la fois individuelle et collective. L'analyse des réseaux de communication dans la classe montre que si les structures groupales sont liées aux groupes d'affinité, elles sont dynamiques et se recomposent au gré des activités (Huillerey \& Keimbou, 2000).

D'autre part, l'analyse de débats d'idées (situation didactique d'interlocutions discursives où il est demandé aux élèves d'expliciter ce qu'ils ont observé, de discuter des interprétations possibles, puis de formuler des stratégies) montre que la dynamique des groupes évolue au plan sociocognitif de façon différentielle selon la manière dont les élèves co-construisent les savoirs au fil des expériences et des actions en projet formulées successivement. II y a une évolution de la stratégie collective dans le sens où les élèves confrontés à de nouvelles résolutions de problèmes peuvent (1) décrire plus précisément les caractéristiques du rapport de force, (2) formuler des issues possibles en relation avec les actions projetées, et (3) apprécier la dynamique du jeu après un retour à l'action (Chang, Wallian \& Nachon, 2006 ; Nachon, Wallian \& Gréhaigne, 2005 ; Labrude, Musard \& Wallian, 2006). Dans cette perspective sémio-constructiviste, la difficulté consiste à accéder à la manière dont l'élève s'y prend pour apprendre et à comprendre comment s'opère le dialogue entre la pensée et l'action. Les premiers débats, dépourvus de spécificité technique, laissent progressivement place à un discours descriptif et argumentatif. En sports collectifs, les configurations du jeu constituent un support intéressant pour le débat d'idées (Gréhaigne, Caty \& Château, 2005). En acrosport, grâce 
à une distanciation progressive par rapport à l'action, des élèves observateurs, peuvent progressivement élaborer les règles de l'action efficace (Musard \& Léziart, 2000).

Par ailleurs, le rôle déterminant des interactions langagières dans les apprentissages en EPS est également mis en évidence à travers une autre orientation théorique, celle de la psychologie sociale. C'est le cas de l'analyse des interactions verbales de dyades en situation de co-construction de règles d'action au handball (Darnis, Lafont \& Menaut, 2006). Le progrès dans l'interaction paritaire entre deux sujets amenés à coopérer dans la construction de compétences tactiques en sports collectifs semble lié à plusieurs facteurs (dynamique interactive riche et variée afin de solliciter des remises en questions chez les partenaires; pertinence des règles d'action; prise en compte de l'adversaire dans l'algorithme d'attaque). Les chercheurs montrent aussi que la confrontation entre pairs débouche rarement sur des oppositions génératrices de conflits sociocognitifs (Lafont \& Proeres, 2000) et que la formation des tuteurs est pertinente dans la mesure où ceux-ci font preuve d'une meilleure sensibilité aux besoins des tutorés et diversifient davantage leurs modes d'intervention (Cicero \& Lafont, 2005 ; Viala \& Lafont, 2005). Sont également étudiées les conditions favorables au managérat d'un pair (en tennis de table, Ensergueix \& Lafont, 2005), les effets de différents modèles (adulte vs enfant) (Lafont, 2005) et le tutorat interculturel (Abdoul Rassoul \& Darnis, 2008).

Enfin, Mascret (2008) propose de favoriser les interactions entre élèves au badminton ( « joueur-coach ») dans le but de développer les apprentissages moteurs en passant d'un coaching émotionnel à un coaching mêlant émotions et conseils adaptés.

\section{Genre et mixité}

Tout d'abord, les pratiques des enseignants face à la mixité sont analysées (Patinet, 2005) en prenant en compte plusieurs variables liées à l'enseignant, au milieu d'exercice et aux caractéristiques de la classe. Globalement, l'enseignement en mixité décline de la $6^{\mathrm{e}}$ à la terminale (de $95 \%$ à $60,5 \%$ ) ; le contexte local et la politique académique apparaissent comme les variables les plus déterminantes (Cogérino, Terret \& Rogowski, 2005). La majorité des élèves est favorable à la mixité (Gagné, Martel et Nadeau, 2005).

Les chercheurs observent que l'impact d'un enseignement est différencié selon le genre des élèves (Cogérino, 2002). D'ailleurs, plusieurs études mettent en évidence l'émergence de contrats didactiques différentiels en EPS, selon le genre des élèves et leur niveau d'habileté. Selon Schubauer-Léoni (1996), le contrat didactique n'est pas négocié uniquement entre l'enseignant et les élèves, mais entre l'enseignant et des sous-groupes d'élèves. La différence des sexes se co-construit et se structure dans les interactions didactiques (El Andoulsi, Bedhioufi \& Amade-Escot, 2006 ; Costes \& Amade-Escot, 2005 ; 
Uchan 2002). Plusieurs facteurs tels que le sexe, les représentations des élèves, l'attitude scolaire, le sens donné aux tâches, la coopération filles -garçons, l'expérience extrascolaire... influencent les interactions didactiques ou expliquent la présence de contrats didactiques différentiels (Verscheure \& Amade-Escot, 2005). Les élèves négocient donc de façon différentielle les exigences des tâches en fonction des effets de contexte (la coopération filles -garçons) et de ces arrière-plans.

D'autres recherches analysent l'influence du genre du professeur sur l'enseignement en EPS (Mauffrey \& Poggi, 2006 ; Thorel \& David, 2006). Les professeurs d'EPS choisissent leurs activités, initient des choix didactiques, développent des comportements avec leurs élèves, qui s'expliquent pour partie par leur identité de genre (Couchot-Schiex, 2006). Les contenus d'enseignement révèlent une hétérogénéité et une visibilité ténue des effets de genre, de même que les formes de groupement. En revanche, les valeurs transmises, le climat affectif et l'expression de l'autorité dévoilent des effets de genre plus marqués.

Les stéréotypes masculins / féminins en EPS pèsent largement sur les modalités d'intervention des enseignants (Coltice \& Cogérino, 2002). L'EPS renforce les inégalités filles - garçons (Couchot-Schiex, Trottin \& Cogérino, 2008), que ce soit par le biais du rapport au corps (Moniotte \& Poggi, 2006) ou par les effets des différentes formes de mixité (Moreno, 2006). Les choix didactiques des enseignants conduisent alors à limiter les apprentissages techniques, tactiques et réglementaires bénéfiques aux filles (Vigneron, 2002 ; Costes \& Deutz d'Arragon, 2002). D'ailleurs, les écarts de notation entre filles et garçons sont significatifs, mais les enseignants ne sont pas conscients des effets de certains facteurs, tels que la programmation des APSA ou le sexe de l'enseignant (Dechef \& Cogérino, 2005) et leur attention pour une plus grande équité est souvent captée par d'autres préoccupations en classe (Patinet \& Cogérino, 2008).

\section{Savoirs professionnels}

Les recherches se proposent d'analyser les conceptions des enseignants relatives à l'EPS (Marsault, 2000) ou à différentes APSA telles que l'escalade (Marchal, Delachauw \& Lab, 2006), la lutte sénégalaise (Wane, Bouthier \& David, 2006), le volley-ball (AmansPassaga, 2005 ; Vercruysse, André \& Carlier, 2008), la course de longue durée (Chaumont, Carlier \& Dervaux, 2005), la danse (Vandesande, Carlier \& Dervaux, 2005), le basket-ball (Deriaz \& Poussin, 2000 ; Fréderic, Delhaes \& Cloes, 2006). Par exemple, trois types de représentations de la gymnastique sont identifiées : une pratique dite artistique (dominante), une pratique dite acrobatique et une pratique dite mixte (Abdi \& Aubert, 2000). 
D'autres études se centrent sur les représentations des enseignants, à propos de sujets divers : l'apprentissage moteur (Araya-Cortez, 2005 ; Chang, Mahut \& Gréhaigne, 2002), la réussite des élèves (Boudreau, 2005), les situations considérées comme injustes par les élèves (Martel \& al., 2000), les accidents scolaires et risques encourus (Thomas-Bion \& Fiard, 2008).

L'hétérogénéité des théories d'action des professeurs est soulignée par Carnel et Delhemmes (2000). En effet, les intervenants construisent un rapport personnel aux APSA selon leurs rapports aux institutions (Ben Jomaa \& Terrisse, 2008; Devos-Prieur \& Amade-Escot, 2005 et Garnier \& Goirand, 2000 en basket-ball; Montaud, 2008 en danse) et mobilisent différents registres de connaissances (empirique, technique, technologique et scientifique) (Brière-Guenoun \& Amade-Escot, 2005 ; Cizeron \& Gal, 2002), voire même des croyances (Cizeron, Gal \& Clace, 2008) en fonction de la situation éducative et du contexte d'enseignement (Saint Voirin \& Gervais, 2000).

\section{Interactions enseignant-élèves}

Plusieurs recherches étudient le jeu des phénomènes interactifs dans la transmission et l'appropriation du savoir à partir du concept de contrat didactique (Garnier, 2006 ; Thépaut, 2002 ; Musard, Thieblemont \& Caty, 2005). L'émergence de contrats didactiques différentiels est mise en évidence dans plusieurs travaux déjà présentés (voir la thématique " genre et mixité ») (Roesslé \& Loquet, 2006 ; Uchan 2002 ; Costes \& AmadeEscot, 2005 ; El Andoulsi, Bedhioufi \& Amade-Escot, 2006 ; Verscheure \& Amade-Escot, 2005). La multiplicité des contraintes agissant sur le système didactique poussent les enseignants à modifier fréquemment le contrat didactique, en utilisant différentes techniques : les techniques dites chronogénétiques (qui ont pour fonction de faire avancer le temps didactique); les techniques dites mésogénétiques (qui relèvent de l'aménagement du milieu) ; les techniques dites topogénétiques (qui visent le partage des tâches et des responsabilités de chacun vis à vis du savoir enseigné). Les interactions didactiques conduisent fréquemment à une réduction des savoirs programmés, voire à une transformation des contenus enseignés.

Les chercheurs analysent les interactions entre enseignant et élèves à partir d'autres outils. Dans une approche phénoménologique, l'espace entre l'enseignant et ses élèves, à un moment donné de l'inter-action, est défini comme un tout indissociable entre engagement corporel et signification. L'inter-action ne se fonde pas que sur la topographie, mais sur les traits signifiants de l'espace pour les acteurs en fonction de leurs engagements à un moment donné (Rix, Biache \& Léziart, 2000). Ces «zones de rencontre » entre enseignants et élèves constituent de véritables rituels et sont le lieu de 
construction de compétences professionnelles (Baeza et Bouthier, 2006). Lémonie, David et Gouju (2006) considèrent les interactions didactiques comme constructions d'un espace partage. En effet, les actions de l'enseignant sont orientées vers la construction et le maintien d'un espace partagé avec l'élève à propos d'un contenu d'enseignement, susceptible de favoriser les apprentissages. Les résultats montrent qu'au-delà d'une compréhension externe de la conduite de l'élève, c'est l'adoption par l'enseignant d'une attitude empathique visant à adopter la perspective de l'élève qui semble permettre la création de cet espace partagé. Les chercheurs mettent en évidence le caractère négocié et co-construit des connaissances (Huet \& Saury, 2008 ; Vellet, 2006) et les effets des négociations sur la motivation des élèves (Dupont, Gérard, Delens \& Carlier, 2008). Ainsi, les règles du travail scolaire sont davantage intégrées par les élèves lorsqu'elles sont coconstruites au cours des interactions et négociées, ou rapportées explicitement par le professeur à un ensemble de buts articulés les uns aux autres (Méard, Bertone, Euzet \& Ria, 2000 ; Petit, Durny, Coulomb, Melet, Pasco, Guibald \& Le Bastard, 2000).

\section{Planification}

L'analyse croisée des curricula (formels, réels ou potentiels) montre que les enseignants ne transposent complètement les pratiques sociales de référence, mais ils composent les pratiques scolaires sous diverses influences, notamment celle des élèves et de leurs collègues (publications, stages de formation continue...) (dans le cas de l'acrosport, Musard, Robin, Mahut \& Gréhaigne, 2002 et du cirque, Mandy Destailleur \& Poggi, 2006). L'organisation de l'équipe EPS joue un rôle décisif dans les choix curriculaires (par exemple dans l'application de nouveaux programmes pour les associations sportives (Briot, 2000); dans l'analyse du travail à l'aide d'une pratique réflexive (Lecuivre, 2006)). Néanmoins, Borges \& Rondeau (2008) mettent en évidence des difficultés liées à la collaboration entre enseignants québécois (clivages disciplinaires, organisation du travail scolaire, surcharge de travail...). Le rôle joué par les directeurs d'école vis-à-vis de l'EPS (Mees, Carlier \& Renard, 2000) ou du sport scolaire (Montreuil, Lacroix \& Trudel, 2006) fait également l'objet de recherches.

Plusieurs études s'intéressent aux décalages entre les pratiques effectives et les textes officiels (Hadjb Taieb \& Hentati, 2008 ; Machemehl \& Lefèvre, 2006). Bouslama (2008) analyse le curriculum souhaité, imaginé par les enseignants d'EP tunisiens, bien distinct du curriculum formel. Les enseignants sont davantage focalisés sur les objectifs généraux de l'EPS, au détriment des objectifs spécifiques des APSA. Ce qui est réellement fait en EPS repose avant tout sur les conditions de pratique et les conceptions des enseignants, bien avant de s'inscrire éventuellement dans les programmes (Léziart \& Pasco, 2006). 
Plus précisément, les enseignants mettent en œuvre une double stratégie lors de l'élaboration des contenus: une stratégie adaptative, qui consiste à sélectionner des contenus compatibles avec les réalités locales (matérielles, sociales, culturelles...) et une stratégie interprétative, qui consiste à donner une légitimité aux structures locales de réflexion curriculaire (Briot, 2002).

Saker \& Cloes (2008) soulignent la variabilité des conceptions de l'EPS selon le contexte culturel: par exemple, les enseignants algériens sont plus centrés sur les aspects " Condition physique ", " Technique et tactique ", " Intégration " et " Entraide ", alors que les professeurs belges retiennent plus fréquemment la catégorie " Santé ". Cette variabilité est également présente au sein d'un même pays. En France, l'analyse des curricula d'EPS révèle également des inégalités sociales selon les types d'établissements scolaires. L'EPS échoue à transmettre une culture corporelle commune au collège (Poggi-Combaz, 2002). En outre, certaines APSA comme le volley-ball ou groupes d'APSA, notamment les APPN sont plus rarement programmées dans les établissements défavorisés (Poggi-Combaz \& Mahut, 2002 ; Ridacker \& Poggi, 2006).

D'autre part, en amont de l'interaction, les chercheurs étudient aussi la catégorisation et la mémorisation des élèves chez les enseignants. Si les enseignants mémorisent davantage les garçons, cette proportion varie significativement avec l'expérience professionnelle. Les élèves sont d'abord mémorisés à partir de leurs comportements conformes aux stéréotypes (garçons perturbateurs et en réussite par exemple), avant de l'être pour leurs performances, réussites ou ressources en liaison avec la discipline (Cogérino \& Lounana, 2006).

\section{Evaluation}

Tout d'abord, l'action de l'enseignant pendant la séance d'évaluation se partage entre la poursuite de différentes intentions (noter le plus justement possible chaque prestation d'élèves, aider chacun d'entre eux à comprendre leur note et leur proposer des apprentissages nouveaux) et une préoccupation plus collective de maintien de la discipline dans la classe (Durny, 2006). Confrontés à une recherche d'équité et d'objectivité, les enseignants sont donc partagés entre leur propre définition de l'évaluation équitable et celle préconisée par les textes officiels. Ils justifient leurs arrangements évaluatifs, permettant de résoudre les contradictions entre valeurs visées, souci d'objectivité et contraintes de faisabilité, en invoquant la norme du mérite et se préoccupent davantage de justice distributive, contrairement aux textes officiels valorisant une justice procédurale (Cogérino\& Mnafakh, 2008). Pour Grenier \& al. (2008), l'évaluation authentique pourrait, par son intégration au moment de l'apprentissage/enseignement, contribuer à réduire le 
temps consacré uniquement à l'évaluation. Par son exigence de pertinence sociale, elle permettrait à l'éducation physique de gagner en prestige auprès des parents et finalement, par l'importance mise sur la signification de la tâche d'évaluation, susciter l'intérêt des élèves et les inciter à s'engager encore plus activement dans les activités d'apprentissage. Davantage d'échanges entre élèves, parents et enseignants permettraient d'améliorer les pratiques évaluatives (Grenier, Raymond, Bégin, Lauzon, Verret et Gagné, 2006).

Les auteurs cherchent à construire une méthode efficiente de constructions de barèmes tout en limitant les décisions et les arbitraires, responsables de multiples arrangements évaluatifs (Cleuziou, 2000). Plusieurs outils d'évaluation sont ainsi expérimentés et proposés (le logiciel CoopVidéoGraphe EPS (Barthès, 2006); une évaluation certificative en course pédestre de durée pour évaluer la $5^{\mathrm{e}}$ compétence culturelle des programmes des lycées (Baudet \& Carnel, 2005); des instruments d'évaluation en acrosport (Caty \& Gréhaigne, 2000) ou en sports collectifs (Zerai \& Bedhioufi, 2008); une évaluation des savoirs conceptuels (Ayers \& Hawkins, 2002).

D'autre part, les sports collectifs sont largement représentés dans les menus d'APSA programmées pour le baccalauréat, conditionnés par les impératifs matériels et la cohérence des enseignements. Quant aux choix des élèves, ils ne semblent pas dépendants du sexe ou de la série du baccalauréat (Cleuziou, Brau-Antony \& David, 2002). Enfin, les référentiels d'évaluation permettent de révéler les conceptions des enseignants d'EPS et de suggérer des pistes de formation. Par exemple, l'analyse des référentiels au baccalauréat de volley-ball fait émerger deux tendances lourdes : une tendance formelle, focalisée sur un ensemble de manifestations gestuelles calquées sur le modèle de la haute performance et une tendance fonctionnelle, axée sur les choix tactiques des joueurs et prenant en compte les possibilités initiales des élèves (BrauAntony \& David, 2000). A noter que la problématique des écarts de notes selon le genre a déjà été évoquée dans la partie " genre et mixité ».

\section{2. APSA étudiées}

La figure 3 présente les activités physiques sportives et artistiques (APSA) étudiées dans les recherches portant sur l'EPS. 


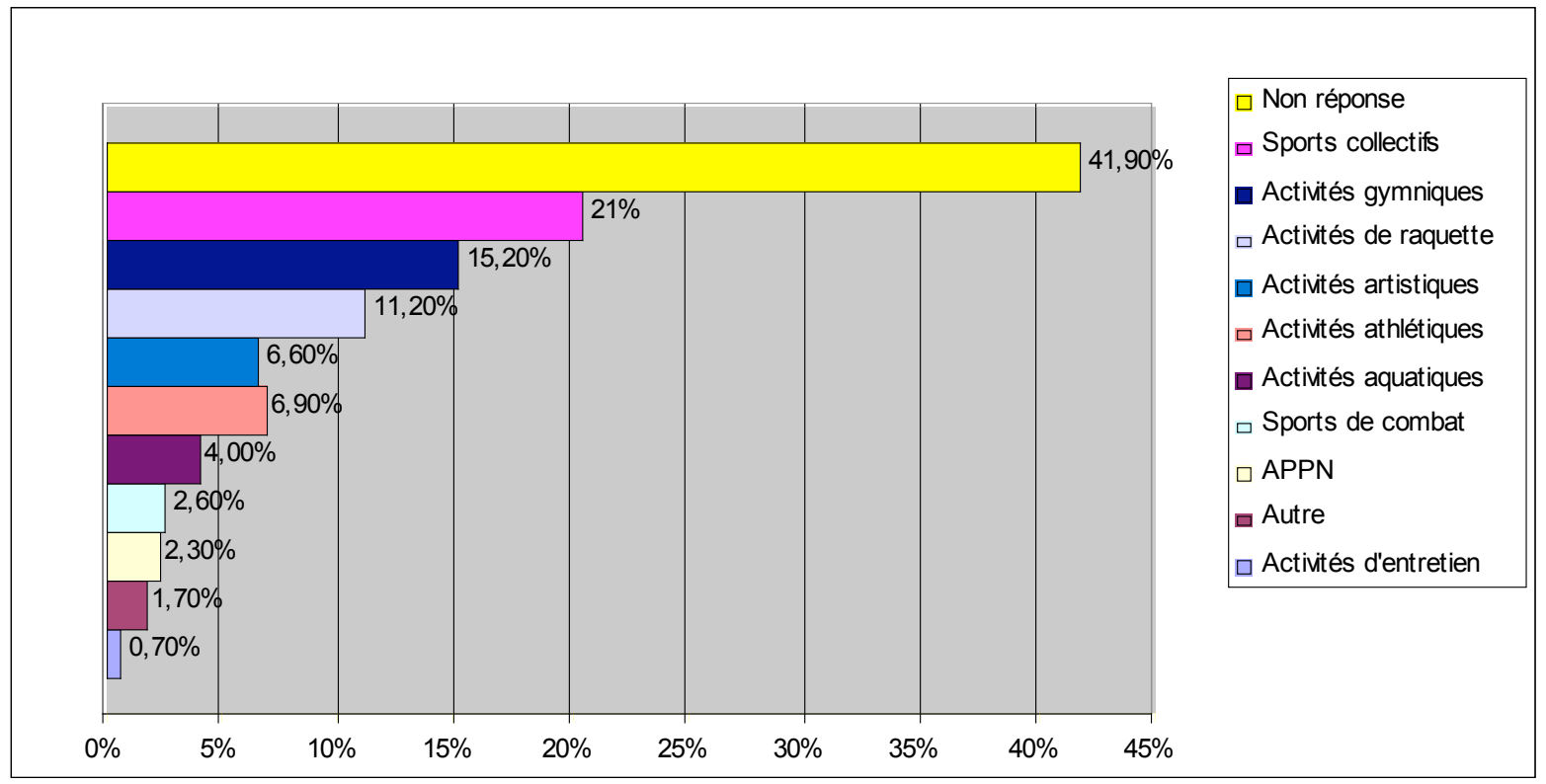

Figure 3. Familles d'APSA étudiées dans les recherches portant sur l'EPS

Nous pouvons expliquer la part importante de non réponses $(41,9 \%)$, c'est-à-dire les réponses où l'APSA support n'est pas précisée, par les raisons suivantes :

- les résumés des recherches issues des programmes scientifiques sont synthétiques et ne présentent pas toujours en détail le contexte d'intervention étudié ;

- certaines enquêtes ne prennent pas en considération les APSA pratiquées par les élèves, car elles s'intéressent à des objets plus larges, tels que les programmes, la santé, les inégalités de réussite...

Lorsque les APSA sont explicitées dans les résumés, soit les chercheurs précisent le contexte d'intervention (APSA, établissement, classe..) pour situer l'étude, soit ils choisissent de se centrer sur des problèmes spécifiques à une APSA, selon leurs centres d'intérêt ou compétences.

Force est de constater une importante disparité dans les familles d'APSA étudiées en EPS, alors que les élèves sont censés développer une pratique polyvalente, tout au moins au collège. Ainsi, les sports collectifs, les activités gymniques et les activités de raquette sont largement plus étudiés que les autres groupements (plus de 10\%). Au contraire, certaines familles comme les activités aquatiques, les sports de combat, les activités physiques de pleine nature (APPN) et les activités d'entretien semblent mises à l'écart (moins de $5 \%$ des recherches). Nous observons donc un décalage entre les APSA étudiées dans les recherches et celles enseignées à l'école. Par exemple, les activités artistiques (encore peu enseignées, sont plus étudiées que les activités aquatiques (6,6\% contre $4 \%$ ). Ce choix d'APSA étudiées dans les recherches peut s'expliquer par divers 
facteurs : thématiques de recherche des laboratoires, compétences, spécialités et intérêts des chercheurs, opportunités et faisabilité des protocoles...

\section{Les thématiques de recherche sur la formation}

Avant de décrire les différentes thématiques des recherches sur la formation, précisons que plus de la moitié des travaux portent sur la formation initiale des enseignants d'EPS (et plus rarement sur la formation des professeurs des écoles et des entraîneurs). Assez peu d'études s'intéressent à la formation professionnelle continue $(7,9 \%)$ ou à la formation des formateurs $(2,4 \%)$.

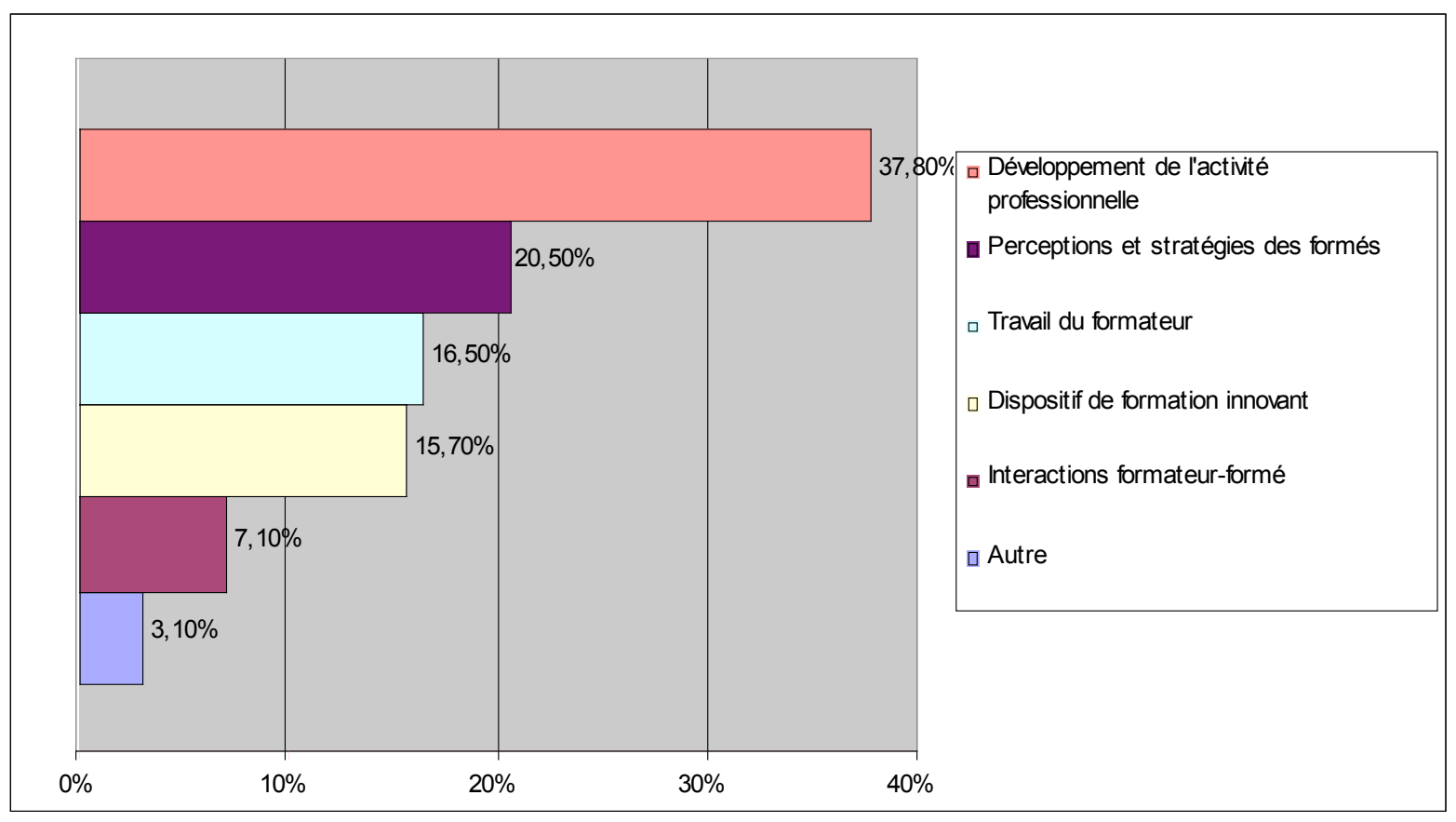

Figure 4. Thématiques des recherches sur la formation

Cinq thématiques d'études émergent de l'analyse a posteriori des résumés (figure 4). Comme pour le contexte d'intervention précédent, l'EPS, nous n'observons pas de différence significative selon les congrès, de 2000 à 2008.

Alors que les études consacrées à l'EPS sont davantage centrées sur l'enseignant que sur l'élève, les recherches sur la formation portent quant à elles plus sur le formé (environ $58 \%$ : « développement de l'activité professionnelle » et «perceptions et stratégies des formés ») que sur le formateur (16,5\%: "travail du formateur »). Les chercheurs, notamment ceux qui forment les futurs enseignants d'EPS en IUFM, souhaitent analyser et comprendre comment les étudiants construisent les compétences professionnelles, et quelles sont leurs perceptions et leurs stratégies face à la formation. Ces connaissances 
semblent en effet essentielles pour alimenter la réflexion sur la formation des enseignants. Enfin, 15,7\% des recherches visent à améliorer la formation des stagiaires à travers des dispositifs innovants et $7,1 \%$ se centrent sur les interactions entre formateur et formés.

\section{Développement de l'activité professionnelle}

De nombreuses recherches analysent l'impact de la formation sur le développement de l'activité professionnelle des formés (Albuquerque, Batista \& Pinheiro, 2006; Alem, Kpazai, Sane \& Couture, 2008 ; Chaliès \& Flavier, 2002 ; Flavier, Bertone, Chaliès \& Méard, 2006 ; Grassetie \& Bouthier, 2006 ; Le Bas, 2002 ; Theunissen, Langer \& Cloes, 2008). Elles décrivent comment les stagiaires s'approprient progressivement les règles constitutives du métier ou les concepts clés, en se centrant d'abord sur les problèmes de gestion de classe au détriment de la dimension didactique (Lenzen, Denervaud, Poussin, Renggli \& Dubbach, 2008). L'analyse de l'expérience vécue d'enseignants d'EPS débutants, source d'apprentissage se caractérise par une forte composante exploratoire et émotionnelle. Les stagiaires construisent des connaissances en enseignant, par exploration, test, puis vérification sur (1) les agissements des élèves, (2) le temps pour agir et (3) la gestion pédagogique (Saintvoirin, Gal \& Durand, 2005). Ces connaissances présentent quatre propriétés selon Ria et Gal (2002). Elles se construisent : a) à l'occasion d'expériences « critiques » ou déstabilisantes ; b) selon un processus de typicalisation, c) parfois sur la base d'une expérience unique ; d) leur degré de validité fluctue en fonction de leur efficacité circonstancielle. Ainsi, les enseignants apprennent à « typifier » l'activité d'enseignement, c'est-à-dire à reconnaître progressivement des situations typiques de classe sur la base d'indices perceptifs récurrents construits in situ et ils construisent des gestes professionnels à partir d'expériences singulières et locales (Gal \& Ria, 2002). Ainsi, Flavier et Chaliès (2006) mettent en évidence la capacité d'un enseignant novice exerçant en zone d'éducation prioritaire (ZEP) à s'émanciper des techniques que lui offre son conseiller pédagogique et à opérer une « migration fonctionnelle » (Clot, 2003) des règles du métier dans d'autres contextes, pour d'autres usages.

Cette construction professionnelle chez les stagiaires ne concerne pas seulement leurs savoirs, mais aussi leurs préoccupations, leurs émotions et leurs façons d'interpréter les évènements en classe. L'engagement des jeunes enseignants, c'est-à-dire leur façon d'éprouver corporellement, cognitivement et culturellement la classe, est caractérisé comme indéterminé et anxiogène, contradictoire et exploratoire (Ria \& Sève, 2002). Ainsi se succèdent des périodes de stabilité et d'instabilité professionnelle, des moments de réinterrogation du métier (Rouve \& Ria, 2008). Mais l'émotion peut aussi constituer un ressort pour l'action professionnelle, car les situations d'enseignement offrent au 
professeur une multitude de possibilités de faire varier son implication affective afin de les rendre plus conforme à ses attentes (Desigaux, Sève \& Adé, 2005). De même, les objets utilisés par les enseignants (fiches de travail, fiches d'évaluation, aménagement matériel...) pour réduire l'incertitude et mieux contrôler la classe peuvent être considérés comme des artefacts du développement professionnel (Adé, Sève, Serres \& Ria, 2005).

D'autres chercheurs suivent tout au long de l'année de formation le processus de construction des compétences professionnelles des stagiaires (Ouitre, 2006 ; Perez-Roux, 2008 ; Ubaldi, Berthier \& Alin, 2006), et soulignent le poids de la formation initiale et de I'histoire sportive sur la relation des enseignants aux savoirs et la diversité des trajectoires professionnelles (Roux-Perez, 2005). Cette construction identitaire s'élabore progressivement à travers la multiplicité et la complexité des expériences vécues (Gautron \& Cogérino, 2002). Par exemple, les différents moments de la formation initiale (rédaction d'une dissertation, stage professionnel, atelier d'analyse de pratiques...) sont propices à la mise en relation d'expériences diverses et permettent aux enseignants de créer une unité entre des savoirs restés auparavant diffus (Serres, Ria, Adé \& Sève, 2005). La technique de l'instruction au sosie (méthode d'auto-confrontation) peut constituer, pour le stagiaire, une occasion de développement de l'expérience, c'est-à-dire à la fois de ses manières d'agir et de penser son travail. Elle permet une prise de conscience qui est à la fois une nouvelle représentation de l'objet et une nouvelle représentation de soi et de ses pouvoirs d'agir (Barbot \& Saujat, 2000; David, Amarouche \& Choffin, 2000). Par exemple, concernant la formation à l'évaluation, cette dynamique de changement s'incarne dans une bascule d'un genre professionnel plutôt centré sur la technicité et la mesure, vers un genre plus clinique ancré sur la volonté de transformer son rapport aux élèves et au métier, en gagnant en souplesse et en adaptabilité.

Un certain nombre de recherches s'intéresse aux problèmes de gestion de la discipline (Desbiens \& al, 2005 ; Bertone, Euzet, Méard \& Flavier, 2000). D’une manière générale, plus un enseignant est débutant, plus il contrôle sa classe, et plus il acquiert de l'expérience en situation de pré-professionnalisation, plus il rend ses élèves autonomes (Vanlerberghe, 2008). Le jeu d'influences concurrentes, telles que la formation initiale, l'observation du conseiller pédagogique, le maintien de la discipline, la réalisation du contenu produit des conflits intrapsychiques chez les enseignants novices dans leurs interactions avec les élèves. Les dilemmes des enseignants débutants présentent certaines spécificités (Ria, Bertone, Méard \& Euzet, 2000): ils sont de types organisationnel, relationnel et curriculaire. Les novices expriment des sentiments d'inconfort lors de ces dilemmes et cherchent à les résoudre en se focalisant sur une 
seule de ces préoccupations. Les auteurs proposent alors des pistes pour aider les enseignants à "déconstruire leur dilemme ». Les situations de conflit, bien que vécues « négativement » in situ, apparaissent a posteriori, formatrices pour les enseignants débutants (Flavier \& Durand, 2002). Elles sont l'occasion d'une contextualisation des connaissances professionnelles acquises antérieurement et d'un approfondissement des savoirs d'action. D'autre part, elles concourent à une prise de conscience de l'imprévisibilité des situations de classe et à la construction de configurations évènementielles typiques. Enfin, elles constituent le point de départ pour des pratiques d'analyse réflexive, sources de développement professionnel.

D'autres recherches se focalisent sur des objets d'étude précis, relatifs à l'intervention des enseignants novices, comme la planification (Bennour, 2008 ; Daele, Carlier, \& Dervaux, 2005), les stratégies de contrôle et de régulation par rapport aux planifications (Vandercleyen, Carlier \& Delens, 2008), la gestion du temps (Azaïs, Cizeron \& Gal, 2002), l'analyse des comportements différenciés des élèves (Coltice, Couchot-Schiex \& Cogérino, 2006) ou encore le questionnement destiné aux élèves (Gal \& Cizeron, 2005 ; Desbiens \& al., 2006). Ainsi, les enseignants stagiaires, focalisés sur leurs propres attentes, laissent peu de temps aux élèves pour répondre, voire même ont tendance à répondre eux- mêmes aux questions. En réalité, ils redoutent ces échanges qui menacent leur autorité.

Peu de recherches s'intéressent à la formation professionnelle continue (FPC) (Lecomte, Carlier \& Renard, 2000 ; Fischback \& Cloes, 2005) : quels sont les principes clés d'une FPC de qualité ? Comment les enseignants du secondaire exploitent les stages de FPC ? Pontais (2000) observe les problèmes rencontrés par les professeurs des écoles pour mettre en œuvre des contenus rénovés en gymnastique rythmique (GR) à l'aide de documents didactiques. Plus les enseignantes sont en difficulté pour définir les contenus, plus elles subissent la pression des élèves, plus l'aspect « formel » de la GR prend le pas sur l'aspect «fonctionnel». Nous retiendrons aussi qu'un projet collectif de développement professionnel, des collaborations prolongées et systématiques entre enseignants et chercheurs développées sur la base d'une approche socioculturelle visant à construire des « communautés de pratique " (Wenger, 1998), aident les enseignants à devenir des leaders en terme de curriculum et d'enseignement (Deglau \& O'Sullivan, 2005 ; O’Sullivan, 2005).

Enfin, le développement professionnel est étudié dans deux autres métiers. Munoz \& Mierjewski (2005) font l'hypothèse que l'ex-étudiant STAPS est porteur d'une expérience d'encadrement et d'un rapport au corps, qui facilitent sa préparation au métier de maître 
de conférences. Quant à Corneloup (2000), il cherche à comprendre comment on devient alpiniste et guide de haute montagne et montre que le vécu d'expériences infantiles et juvéniles apparaît indispensable dans la majorité des cas, notamment le contact avec la nature, les histoires racontées, les livres lus, l'initiation et la pratique de l'alpinisme.

\section{Perceptions et stratégies des formés}

Plusieurs recherches s'attachent à comprendre le sens que les étudiants (STAPS, PLC EPS ou PE) accordent à leur formation (Arnaud, Louis \& Refuggi, 2006 ; Cleuziou \& Pontais, 2006 ; Mokhenache, Wallian \& Gréhaigne, 2005 ; Aubert \& Marguet, 2002), et soulignent les difficultés des étudiants face aux savoirs théoriques (Baba-Moussa, Rahamane \& Garncarzick, 2006) et le sentiment d'efficacité élevé pour gérer la classe (Desbiens, Roy, Spallanzani, Brunelle, Turcotte \& Lanoue, 2005). D'autres visent à identifier les représentations des étudiants à propos de l'EPS (plus précisément le métier d'enseignant d'EPS (Gojard \& Terral, 2008), les objectifs et valeurs de l'EPS (Graça, Bothelo-Gomes, Silva \& Queiros, 2005), les activités sportives (la GR pour Ferrand \& Hevig, 2006 ; le tennis pour Nunes, Beltrao, Dos Santos \& Boga Filho, 2006 ; les jeux sportifs collectifs pour Cordoba, Dénervaud, Deriaz, Lenzen \& Poussin, 2008), l'évaluation en primaire (Grenier \& Kalinova, 2005) ou de la formation (le rôle des conseillers pédagogiques (Albuquerque, Graca \& Januario, 2000), la formation que devrait recevoir l'enseignant d'EPS spécialisé (Redon, 2000). Enfin, les perceptions des étudiants sont également analysées à travers le cours d'action dans le cadre de pratiques sportives (la GR pour Blocher \& Durny, 2005 ; la course d'orientation pour Testevuide, 2000).

Un autre volet des recherches étudie les stratégies d'apprentissage des étudiants, qui réussissent davantage quand ils exercent une pratique sportive régulière (Dupont \& Garcia, 2005). La variabilité des stratégies estudiantines est mise en avant, que ce soit dans le domaine de la gestion corporelle (De Fabrique \& Cogérino, 2006) ou du coping dans le cadre de la gestion du stress sportif (Carton, 2005). D'autre part, Martin et Lafont (2005) démontrent que les étudiantes en difficulté progressent davantage dans la réalisation de l'appui tendu renversé avec un « coping model » (modèle ajusté au niveau de compétence des apprenants) plutôt qu'avec un « modèle de maîtrise » (expert). Quant à Vanlerberghe et Bui-Xuan (2005), ils constatent, par la technique des associations libres, que l'expertise des étudiants STAPS de $1^{\mathrm{e}}, 2^{\mathrm{e}}$ et $3^{\mathrm{e}}$ année est corrélée à leur expérience pédagogique (étape émotionnelle, étape fonctionnelle, étape technique). Enfin, Bolatre et Carnel (2005) s'intéressent à l'épreuve vidéo de l'agrégation externe d'EPS et mettent en évidence un décalage entre les attentes des jurys et les compétences manifestées par les candidats. 


\section{Travail du formateur}

Les chercheurs manifestent un intérêt pour caractériser et comprendre le travail réel du formateur (Leriche, Desbiens, Dugal, 2008 ; Saujat \& Laurent, 2008). Ainsi, Alin (2006) identifie, par une analyse empirique, ergonomique, anthropologique et sémiotique des situations de formation (et d'enseignement), douze gestes professionnels des conseillers pédagogiques (et des enseignants), assortis de leurs en-(je)ux symboliques, ainsi que douze obstacles didactiques professionnels (difficultés et points d'ancrage par lesquels passe l'appropriation des gestes professionnels). Les maîtres de stage considèrent qu'ils participent avec efficacité à la formation du futur professionnel, par une aide de proximité un compagnonnage - centrée sur la résolution de problèmes (Carlier \& Vandesande, 2002). L'activité des tuteurs se caractérise par une observation participante et une mise en œuvre de différentes modalités d'aide auprès des stagiaires (immédiate, différée, mixte), dans un cadre de règles d'action visant à respecter les responsabilités de chacun (Chaliès, 2000). Le tuteur incite le stagiaire à construire des seuils d'intelligibilité de son expérience, allant de l'expression de la subjectivité par des énoncés descriptifs et narratifs, à l'expression d'une objectivité par des énoncés explicatifs co-validés (Gal \& Cizeron, 2006). D'ailleurs, la recherche coopérative entre chercheurs et formateurs semble fructueuse (Dugal, 2005), puisqu'elle permet une co-construction de ces savoirs professionnels, grâce à l'interaction analyse de pratiques et appropriation de notions théoriques (principalement celles d'observation didactique, de contrat didactique et de milieu didactique), même si cette appropriation ne se produit pas sans quelques transformations (Terral \& Collinet, 2008). Les enseignants sont amenés à se centrer sur la question fondamentale " qu'apprennent les élèves ? », et passent ainsi d'une observation spontanée à une observation armée, d'un conseil « spontané » à un conseil « réfléchi », " du prescriptif au descriptif », ou encore " de la critique au questionnement ».

D'autre part, il semble également important de se centrer sur la manière dont le maître de stage construit lui-même ses compétences (Carlier, Daele, Chaumont, 2008). En effet, l'intervention des tuteurs évolue tout au long de l'année de formation (Euzet, Méard \& Bertone, 2000) et les conceptions des formateurs se forgent au cours de nombreuses expériences (Albuquerque, Graça, Januario \& Durand, 2002). Cizeron (2006) montre comment les connaissances des formateurs s'organisent en systèmes de croyances particulièrement cohérents. D'ailleurs, les théories d'action didactique singulières des formateurs génèrent des interprétations différentielles du projet de formation institutionnel (Marre, 2008) ou des situations didactiques (Lebouvier, 2006). Par exemple, on peut s'interroger sur la place accordée aux enseignements de technologie des APSA dans les 
différentes formations STAPS (Louis \& Refuggi, 2006) ou dans le discours syndical (Louis, Refuggi \& Attali, 2005).

\section{Dispositifs innovants}

Les chercheurs évaluent des modalités particulières de formation, notamment :

- le développement de relations plus collaboratives entre enseignants novices et conseillers pédagogiques (co-préparation d'une leçon, co-intervention en classe et co-bilan de cette leçon) (Chaliès, Flavier \& Bertone, 2006) ou entre enseignant novice - conseiller pédagogique - formateur universitaire (triades stables tout au long de l'année, Chaliès \& Escalie, 2008). Par exemple, il s'agit de former par la démarche réflexive pour amener le stagiaire à formuler une demande d'aide auprès de son conseiller pédagogique (Cizeron \& Gal-Petitfaux, 2006), ou d'intégrer dans la formation des données issues de la recherche, comme la capacité à réaliser une observation didactique (Brau-Antony, 2006);

- différents programmes de formation (co-construction du programme par des formateurs et des chercheurs dans le but de prendre davantage en compte le curriculum scolaire (Spallanzani et al., 2006); prise en compte des problèmes rencontrés par les professionnels pour construire une formation en judo (Loizon \& Terrisse, 2000); valorisation de la dimension technologique (Refuggi, Louis \& Deridder, 2005); soutien de l'autonomie sur le style motivationnel d'enseignants d'EPS (Tessier \& Sarrazin, 2006) ; communication pédagogique en entraînement sportif (Roy, Beaudoin. Perreault, Turcotte, Spallanzani, \& Desbiens, 2008); entraînement à la prise de décision par une démarche d'autosupervision (Charest, Roy, Perreault \& Brunelle, 2008); développement de la pratique réflexive des élèves en jeux collectifs (Chang, Wallian \& Gréhaigne, 2008);

- Des outils innovants (instrument d'observation pour former les intervenants en rugby, (Ulhrich, Marty \& Bouthier, 2006) ; manuel technologique destiné à la formation des entraîneurs de ski alpin (Roux \& Durey, 2002) ; simulateur de conduite de deux roues (Aupetit \& Oliviero, 2008));

- Des modules de formation à distance (Lenzen \& Cloes, 2005 ; Theunynck, Pezé, Bui-Xuan \& Mikulovic, 2005) ou dans un environnement d'apprentissage multimédia (Leblanc, 2000).

\section{Les interactions formateur - formé}

L'activité collective d'entretien entre le formateur et le formé est évolutive (Chaliès, Trohel \& Durand, 2000 ; Darnis, 2008 ; Serres, 2008) et dépend directement de la nature des interactions entre les conseillers pédagogiques $(\mathrm{CP})$ et les stagiaires. Ces dernières 
s'organisent selon des références identitaires multiples et fluctuantes (Trohel, 2000) et deviennent progressivement plus cordiales et plus propices au partage et à la recherche de l'autonomie (Trohel \& Saury, 2006). Imbert (2006) met en évidence l'existence de cycles de don qui se développent au sein de communautés de pratique. Ainsi, la formation des enseignants pourrait être dépendante, pour partie, de la nature des liens de confiance ou de défiance qui se tissent entre formateurs et formés. Mais Bertone (2002) relativise l'impact de ces interactions de formation sur le développement professionnel des novices, puisque (1) les CP privilégient des interactions visant la protection des stagiaires et la réduction de l'anxiété, au détriment de celles portant sur leurs erreurs et leurs difficultés ; (2) les novices acceptent difficilement de discuter de leurs difficultés avec leurs CP et leurs jugements respectifs diffèrent sensiblement. Quant à Beaulieu, Rivard \& Castani (2008), ils se centrent sur les facteurs favorables et les obstacles au fonctionnement en triade (stagiaire - enseignant - universitaire). Enfin, Kozanitis \& Desbiens (2005) montrent que le nombre d'interactions verbales entre étudiants et enseignant fluctue selon le nombre d'étudiants par classe, le type et la disposition du mobilier et le positionnement spatial des étudiants.

\section{Les recherches sur l'entraînement}

4. 1. Thématiques des recherches

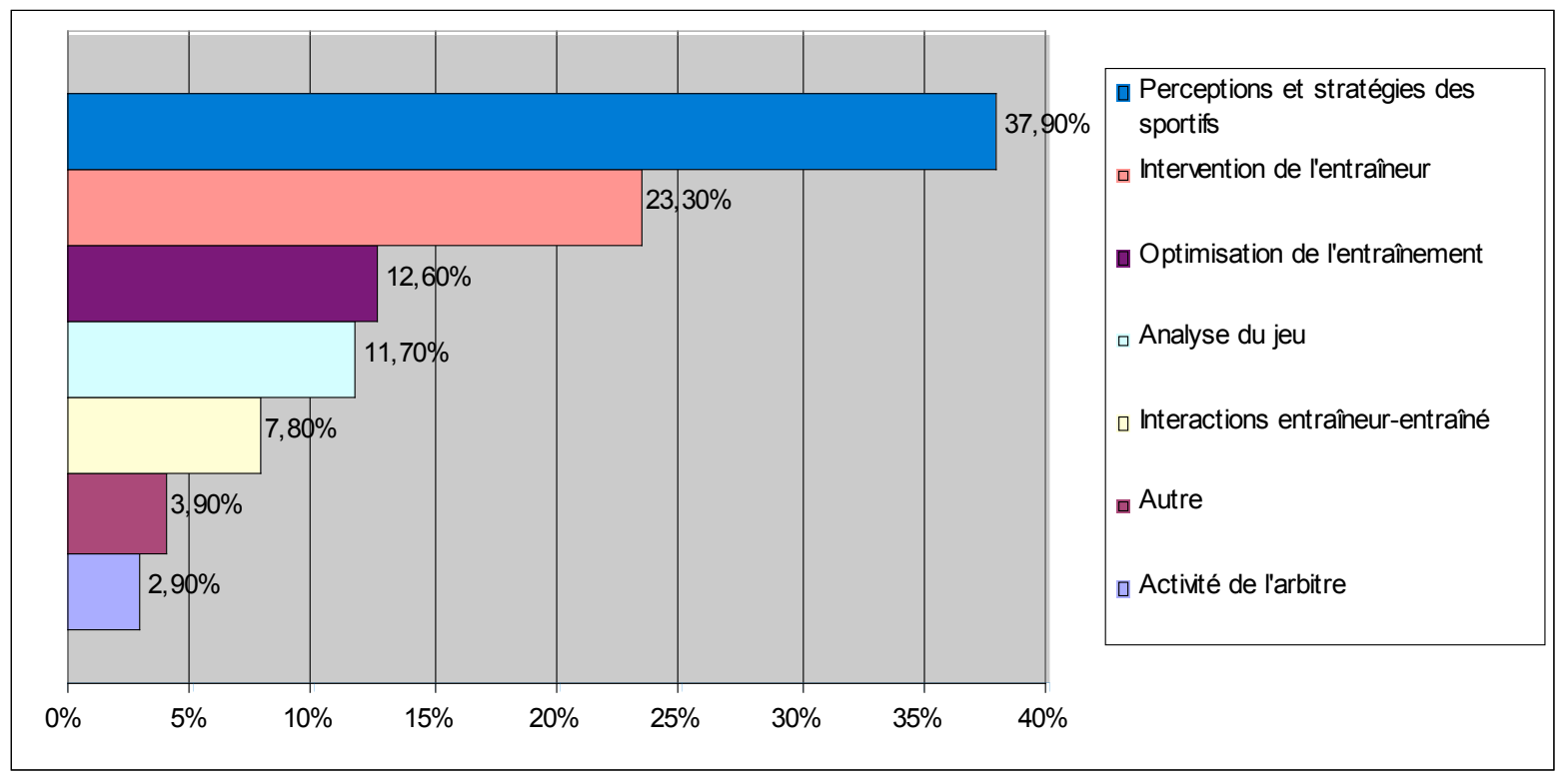

Figure 5. Thématiques d'étude des recherches portant sur l'entraînement 
Dans le domaine de l'entraînement, deux thématiques sont significativement plus étudiées (figure 5) : les « perceptions et stratégies des sportifs » $(37,9 \%)$ et « l'intervention de l'entraîneur " (23,3\%). L'activité des pratiquants est donc plus étudiée que celle de l'intervenant, alors que c'est l'inverse qui se produit dans le contexte de l'EPS : les études centrées sur l'intervenant (enseignant) sont davantage développées que celles qui analysent l'activité des pratiquants (élèves) (voir p. 3). Ensuite, les recherches portent sur «l'optimisation de l'entraînement » (12,6\%), «l'analyse du jeu» (11,7\%) et les " interactions entraîneur-entraînés » $(7,8 \%)$. Enfin, quelques recherches s'intéressent à « l'activité de l'arbitre » $(2,9 \% ;$ Rix, 2008).

\section{L'activité des sportifs}

Les chercheurs ont pour but de décrire et comprendre comment les entraînés vivent et perçoivent leurs expériences dans des sports très variés. Ils identifient alors les structures significatives de l'activité des sportifs et spécifient leurs préoccupations, leurs perceptions et les connaissances mobilisées (Gouju, 2002 en course de haies; Manteau \& Testevuide, 2005 en danse ; Bosseray \& Avanzini, 2000 et Louis \& Refuggi, 2002 en golf ; Fache \& Recopé, 2006 en volley-ball ; Poulin \& Avanzini, en tennis 2000; Launay \& Avanzini, 2000 en karting; Krause \& Saury, 2002 en funboard ; Roy, Trudel \& Lemyre, 2000 ; Muguet, Léziart, Carnel, 2008 en basket-ball ; Poizat, Adé, Gal-Petitgaux \& Seifert, 2008 en natation ; Haise, 2000 en kayak). Quant à Dusson, Riff et David (2006, 2008), ils investiguent l'identité de joueurs de hand-ball ou de gymnastes dans une perspective ergonomique. Les athlètes sont aussi questionnés sur différents sujets, tels que les compétences de l'entraîneur efficace, qui se situent selon eux dans les relations humaines et le savoir être (Walczak, Brunelle, Stoloff \& Desbiens, 2006), les valeurs auxquelles ils sont attachés dès l'adolescence (victoire, effort, travail, volonté, équipe en hockey sur glace, Bernard, Trudel et Marcotte, 2000), ou l'analyse de leur structure de formation (Theunissen, Pauly et Cloes, 2006).

D'autre part, un autre volet des recherches a pour but d'identifier les stratégies des sportifs. Par exemple, comment construisent-ils les savoirs (trier, faire des hypothèses, tirer des principes d'action par une analyse réflexive, profiter des expériences des autres, les mettre en réseau...) ? (voir Peter, 2006 en tennis et Mokhenache, Wallian \& Gréhaigne, 2005 en judo).

En sports collectifs, les prises de décision du coach et des joueuses (Lenzen, Theunissen \& Cloes, 2006) et des passeurs de haut niveau (Eloi, 2008) sont analysées. Cloes, Résimont \& Piéron (2000) montrent d'ailleurs que la mémorisation des interventions de l'entraîneur est limitée pour les joueurs à cause de plusieurs facteurs (problèmes de 
disponibilité mentale, courbe d'oubli très précoce, influence d'autres informations...). D'autre part, les styles personnels des sportifs sont soulignés en rugby par Mouchet (2006) et en danse (improvisation) par Couderc \& Léziart (2002).

D'autres recherches sont menées dans différents sports sur des objets spécifiques, par exemple :

- dans les activités gymniques (l'impact des informations visuelles partielles en gymnastique (Kohler, Stucchi, Papaxanthis \& Pozzo, 2002) ; le phénomène de pertes de figure au trampoline (Robin \& Berthelot, 2002)) ;

- dans les activités athlétiques (les rituels des sportifs: prise de renseignements, progressivité dans la spécificité de la préparation, socialisation, briefing avec l'entraîneur, routines de pré-départ chez les sprinters et placement stratégique sur la ligne de départ chez les coureurs de fond (Lenzen, Delfosse, Menten, Piéron \& Cloes, 2000) ; la régularité d'allure en course de durée, qui ne constitue pas un objectif pertinent (Lab, Marchal et Gréhaigne, 2002)) ;

- dans les APPN (la coopération entre grimpeurs due à la quasi-absence d'entraîneurs aux cotés des grimpeurs (Donzé et Durand, 2000), la collaboration tactique d'équipages experts en voile (Saury et Testevuide, 2002)).

\section{Intervention de l'entraîneur}

Les recherches analysent les comportements des entraîneurs (Franco, Rodrigues \& Castaner, 2006, en fitness ; Vellet en danse, 2005 ; Roy, Desbiens, Spallanani, Lacasse, Lanoue \& Brunelle, 2005 en football américain), en se centrant par exemple sur leurs savoir-faire professionnels (Robin \& Lemonie, 2008 ; Rolland, Cizeron \& Biache, 2008 en gymnastique), leurs intentions (Perreault, Roy, Desbiens \& Harnois, 2006 en volley-ball), ou la gestion des remplacements de joueurs en match (Cloes, Lenzen, Sikora et Pieron, 2000 ; Delrieu, Mouchet \& Bouthier, 2008). En sports collectifs, les pratiques technicistes sont questionnées et comparées avec des pratiques globales (Vallée, Menaut, Lafont \& Bouthier, 2006). Leur importance dans les clubs sportifs belges francophones par rapport au contexte scolaire (Frédéric, Gribomont \& Cloes, 2008 ; Frédéric \& Cloes, 2008) ouvre des perspectives pour la formation des entraîneurs.

D'autre part, les chercheurs étudient les variations des communications de l'entraîneur au cours de la saison sportive (Crispim-Santos \& Rodrigues, 2006 ; Perreault, Roy, Desbiens \& Spallanani, 2006). Ceux-ci adaptent leur intervention en fonction des évènements (Derome, Pirson \& Cloes, 2005 ; Baron \& Loquet, 2002) et manipulent différentes variables didactiques (Loizon \& Terrisse, 2002). D'autres études mettent en évidence que si les entraîneurs utilisent régulièrement internet, ils interagissent très peu avec leurs 
pairs, limitant ainsi la co-construction d'un savoir en entraînement sportif (Lemyre, Trudel \& Durand-Bush, 2006 ; Lemyre, Trudel \& Culver, 2008). Enfin, l'étude des différentes façons de faire des entraîneurs (Morency, Bordeleau \& Léone, 2008) révèle des styles très personnels (Mouchet, 2008).

\section{Analyse du jeu}

Plusieurs chercheurs analysent le jeu en football, plus précisément les configurations typiques du jeu en déviations (Lemoine, 2006), la récupération du ballon (Duprat \& Gréhaigne, 2006), les pertes de ballon (Duprat \& Trimoreau, 2008) et les techniques de frappe de balle (Kervella \& Léziart, 2002). D’autres s'intéressent aux compétences des sportifs (Eloi, 2002 en volley-ball; Le Pallec \& Léziart, 2002 en tennis). Enfin, des chercheurs élaborent des outils d'analyse du jeu (en rugby, Uhlrich, 2005 ; en hockey sur glace, Nadeau \& Poirier, 2006 ; en football, Lecoq, 2008).

\section{Interactions entraîneur-entraîné}

Les études cherchent à élucider la dynamique des savoirs et leur circulation à travers les interactions effectives entraîneur - sportifs (en GR, Loquet \& Gantcheva, 2006 ; en danse, Vellet, 2006 ; en athlétisme, Le Paven, 2008). Carpentier \& Mercier Lefèvre (2006) montrent comment ces interactions, en judo, révèlent des échanges de connaissances, construisent de l'identité, génèrent des significations culturelles et des imaginaires. D'autre part, Hutteau et Avanzini (2000) montrent que la forme de collaboration coach - athlète en canoë s'éloigne du schéma classique prescriptif entraîneur - entraîné en privilégiant un mode interrogatif. Ainsi, le sportif est l'ultime décideur des options technico-tactiques retenues. Mais la relation coach - athlète peut poser problème. Par exemple, des conflits relatifs à la répartition des rôles peuvent émerger: l'entraîneur intervient sur un mode tactico-technique, alors que l'escrimeur attend un soutien affectif (Loriot \& Avanzini, 2000).

\section{Optimisation de l'entraînement}

Ces recherches visent à améliorer l'entraînement (en GR, Gantcheva, Mineva, Loquet \& Léziart, 2008 ; en football, Pehoiu, 2006 ; Demers, 2002). Certains chercheurs aident les entraîneurs de sports collectifs à s'approprier l'entraînement à la prise de décision (méthode qui combine l'entraînement technique, l'entraînement tactique et l'effort physique) (Trépanier, Brunelle, Stoloff \& Desbiens, 2006 ; Roy, Brunelle, Perreault, Desbiens \& Spallanani, 2005) et vérifient l'effet de cette nouvelle approche de l'entraînement sur l'amélioration de la performance des volleyeurs (Loiseau, Brunelle, Lemieux, Roy \& Turcotte, 2006 ; Stoloff, Brunelle, Roy \& Spallanani, 2006). D'autres chercheurs analysent la problématique de l'abandon (« burnout ») et proposent des pistes d'intervention pour les responsables de clubs (en natation de compétition Lefèvre, Habsch 
\& Cloes, 2005 ; en tennis, Moulin, Lafont \& Menaut, 2005 ; dans le milieu du fitness, Laraki, Ancion \& Cloes, 2005). Enfin, Letort (2000) adapte des formats de jeu évolutifs pour la compétition amateur en tennis («Tennis Cooleurs »).

\section{2. APSA étudiées}

Les recherches sur l'entraînement semblent davantage prendre en compte la spécificité des activités sportives. En effet, on compte seulement 4,9\% de non réponses (figure 6). Un déséquilibre important dans les groupes d'APSA étudiés apparaît, puisque presque la moitié des recherches sur l'entraînement se focalise sur les sports collectifs, notamment le football, le volley-ball, le rugby et le basket-ball.

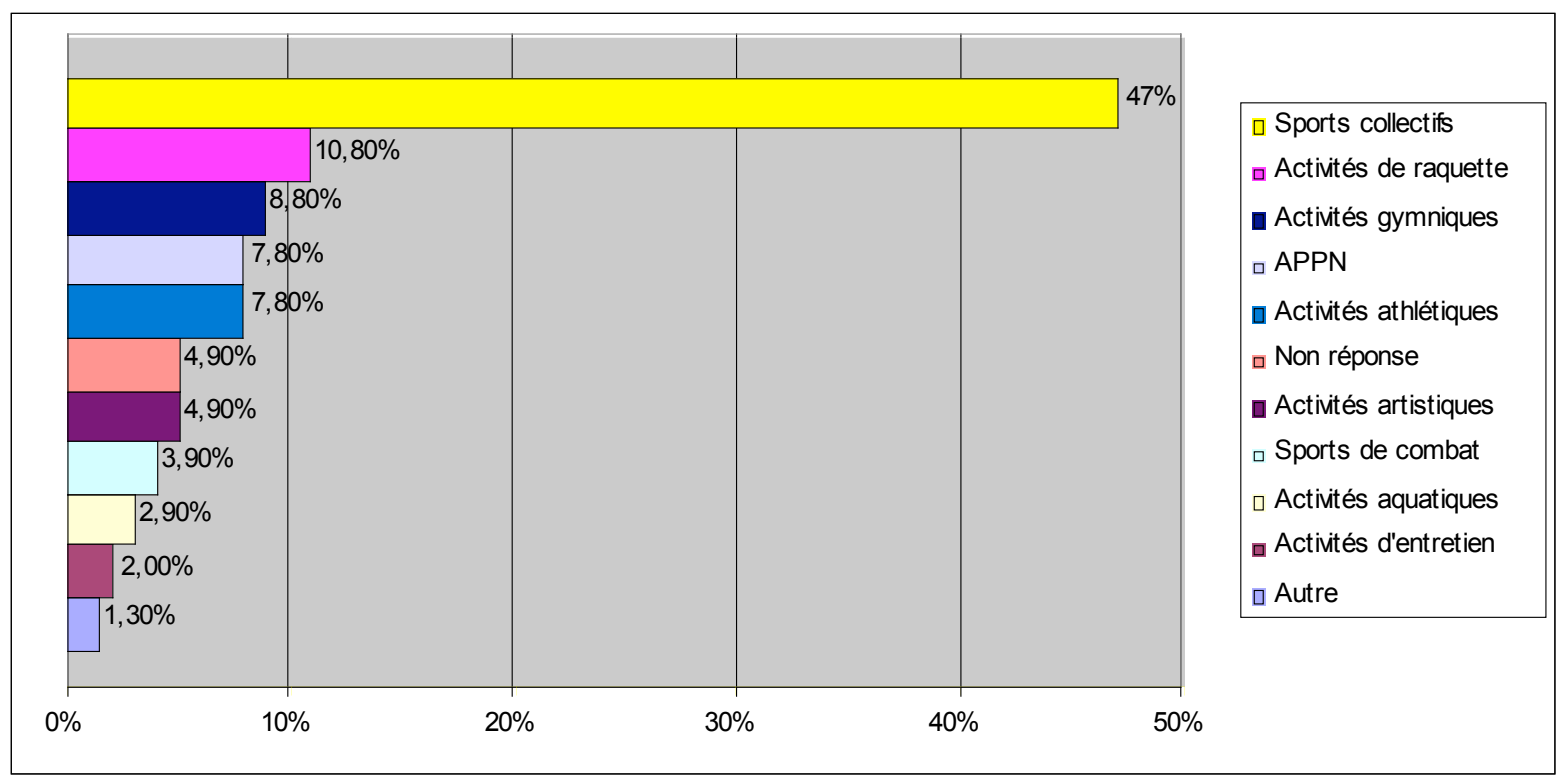

Figure 6. APSA étudiées dans les recherches portant sur l'entraînement

\section{Conclusion}

Cette deuxième partie du panorama des biennales de l'ARIS de 2000 à 2008 s'est centrée sur les recherches, et plus particulièrement sur les contextes d'intervention étudiés et les thématiques développées. Nous n'avons pas observé d'évolutions significatives pour la période 2000 - 2008. La stabilité des résultats peut s'expliquer par le fait que les chercheurs analysent pendant plusieurs années les mêmes objets. En effet, les études sur l'intervention exigent un temps long pour construire le cadre théorique, recueillir des données sur le terrain et traiter les données. De plus, les chercheurs débutent souvent de nouvelles études à partir des résultats obtenus pour produire des connaissances de plus en plus approfondies.

D'autre part, les données présentées devraient permettre aux membres de l'ARIS de se positionner dans le champ de l'intervention en contextes variés et d'engager des 
discussions pour envisager l'avenir de ce domaine en plein essor. Les points suivants méritent d'être débattus :

- le déséquilibre quantitatif des recherches selon les contextes étudiés. En effet, certains contextes sont privilégiés (essentiellement l'EPS, puis la formation et l'entraînement), alors que d'autres semblent délaissés (les APA, les loisirs, l'EPS dans le premier degré, la formation continue) ;

- une unité autour de l'intervention malgré des contextes d'intervention très variés. En effet, il semble utile de développer les points de vue relatifs à l'intervention afin de mieux circonscrire et donner chair à ce concept. Nous constatons la diversité des contextes étudiés, dont dépend l'activité des acteurs qui y sont engagés. Cependant, quelque soit le secteur étudié, les recherches investiguent le même objet, l'intervention en sport à partir des mêmes entrées : soit par l'activité de l'intervenant, soit par l'activité du pratiquant ou de l'apprenant, soit par les APSA, soit par les interactions intervenant - pratiquant (ou apprenant).

- des thématiques de recherche privilégiées selon les secteurs. Ainsi, les recherches en EPS portent en priorité sur les enseignants, alors que les recherches sur la formation et sur l'entraînement sont davantage ciblées sur le formé et le sportif. Les chercheurs s'orientent donc vers la connaissance des perceptions et des stratégies de celui qui apprend, qui cherche à progresser, comme si l'activité du formateur ou de l'entraîneur pouvait passer au second plan. A l'inverse, tout se passe comme si la priorité en milieu scolaire demeurait l'enseignant, qui représente celui qui a le pouvoir et qui reste le maître en matière de décisions et de choix d'intervention ; l'activité des élèves semble alors plus secondaire.

- la variable APSA semble déterminante dans les recherches sur l'entraînement, alors qu'elle semble plus secondaire en EPS (proportion importante de non réponses dans les résumés). En revanche, nous constatons des similarités dans les APSA étudiées. Que ce soit en EPS ou en entraînement, les sports collectifs, les activités de raquette et les activités gymniques occupent le trio de tête, alors que les sports de combat, les activités d'entretien et les activités aquatiques sont marginalisés.

- les spécificités des équipes de recherche. Les analyses factorielles par correspondances mettent en évidence que les laboratoires de recherche se spécialisent dans l'étude de certains contextes d'intervention. Ainsi, certaines universités travaillent plus particulièrement sur l'EPS (Besançon, Lyon, Toulouse, Rennes, Louvain La Neuve, Bordeaux, Orléans, Paris 12, Clermont-Ferrand), d'autres s'orientent vers l'entraînement (Paris 12, Orléans, Rennes, Clermont-Ferrand, Sherbrooke, Liège, Bordeaux, Ottawa, 
Laval, Nantes, Besançon, ...), d'autres encore vers la formation des intervenants (Toulouse, Clermont-Ferrand, Sherbrooke, Nantes, Bordeaux, Lyon, Louvain-La-Neuve, Porto, Grenoble, Caen). Une cartographie de la recherche sur l'intervention en sport pourrait donc être élaborée, car les enseignants-chercheurs orientent les thésards vers les axes de recherche des laboratoires. Par exemple, concernant l'EPS, les interactions langagières entre élèves sont essentiellement étudiées à Besançon et Bordeaux, les questions de genre à Lyon et à Toulouse sur les interactions enseignant-élèves et les questions de genre; les laboratoires de Besançon, Sherbrooke et Montréal sur les curricula.

Cette analyse souhaite donc donner à lire un état des lieux des communications produites au cours des cinq premiers congrès de l'ARIS, de 2000 à 2008, en identifiant les secteurs d'intervention étudiés et en présentant les divers objets d'étude traités dans les recherches sur l'intervention. Au-delà des traces laissées à la mémoire collective, le choix des catégories d'analyse qui président à l'organisation de cet article, a pour but de faciliter les revues de littérature des chercheurs, des étudiants et de leurs formateurs.

\section{Références}

Abdi, G., \& Aubert, J. (2000). Représentations sociales de la gymnastique et pratiques d'enseignement de professeurs stagiaires en E.P.S. Communication affichée présentée lors de la biennale de l'ARIS. Grenoble, 14-16 Décembre 2000.

Abdoul Rassoul, M., \& Darnis, F. (2008). Tutorat interculturel en EPS. Communication affichée présentée lors de la biennale de l'ARIS. Besançon, 9-12 Mai 2006.

Adé, D., Sève, C., Serres, G., \& Ria, L. (2005). Les objets : ressort du développement professionnel. Communication orale présentée lors de la biennale de l'ARIS. Louvain-La-Neuve, 20 -22 Janvier 2005.

Albuquerque, A., Batista, P., \& Pinheiro, C. (2006). Étude de l'évolution des perspectives professionnelles des élèves d'éducation physique et sport pendant sa formation dans les secteurs public et privé. Communication orale présentée lors de la biennale de l'ARIS. Besançon, 9-12 Mai 2006.

Albuquerque, A., Graca, A., \& Januario, C. (2000). Comment les enseignants stagiaires caractérisent-ils leurs conseillers pédagogiques ? Communication affichée présentée lors de la biennale de l'ARIS. Grenoble, 14-16 Décembre 2000.

Albuquerque, A., Graça, A., Januario, C., \& Durand, M. (2002). Comment les conseilllers pédagogiques utilisent dans leur activité de formation leur expérience personnelle 
passée en tant que professeur stagiaire. Communication orale présentée lors de la biennale de l'ARIS. Rennes, 12 -14 Décembre 2002.

Alem, J., Kpazai, G., Sane, O., \& Couture, R. (2008). La prédiction de l'engagement professionnel des étudiants-stagiaires en Education Physique et Sportive à I'INSEPS de Dakar au Sénégal. Communication affichée présentée lors de la biennale de l'ARIS. Rodez, 14-16 Mai 2008.

Alin, C. (2006). Le travail réel du conseiller pédagogique. Communication orale présentée lors de la biennale de l'ARIS. Besançon, 9-12 Mai 2006.

Amade-Escot, C. et al. (sous presse). L'intervention en sport et ses contextes institutionnels : cultures et singularité de l'action.

Amans-Passaga, C. (2005). L'action du professeur d'EPS en situation de managérat à I'Association Sportive : conception des sports collectifs et interactions verbales en contexte de jeu. Communication affichée présentée lors de la biennale de l'ARIS. Louvain-La-Neuve, 20 -22 Janvier 2005.

Anciaux, F., \& Alin, C. (2006). Interactions langagières et alternance codique en éducation physique et sportive aux Antilles françaises. Communication orale présentée lors de la biennale de l'ARIS. Besançon, 9-12 Mai 2006.

Araya-Cortez, E. (2005). Profils socioprofessionnels, modèles disciplinaires et représentations de l'apprentissage moteur chez les enseignants d'éducation physique chiliens. Communication affichée présentée lors de la biennale de I'ARIS. Louvain-La-Neuve, 20 -22 Janvier 2005.

Arnaud, P., Louis, E., \& Refuggi, R. (2006). Quels sens les étudiants de STAPS accordent-ils aux enseignements de technologie des APSA qui leur sont dispensés ? Communication orale présentée lors de la biennale de l'ARIS. Besançon, 9-12 Mai 2006.

Attiklémé, K., \& Refuggi, R. (2002) Approche didactique des conditions cognitives d'élaboration des programmes en EPS : le cas de l'enseignement de la natation. Communication affichée présentée lors de la biennale de l'ARIS. Rennes, 12 -14 Décembre 2002.

Aubert, J., Durand, M., \& Refuggi, R. (2001). L'intervention dans le domaine des activités physiques et sportives : compétence(s) en mutation ? Grenoble : IUFM.

Aubert, J., Barnieri, V., Lamazouere, F., Marguet, L., Perrey, C., \& Wouters, S. (2002). Mémoire professionnel et pratique d'enseignement d'éducation physique et sportive. Communication orale présentée lors de la biennale de l'ARIS. Rennes, 12 -14 Décembre 2002. 
Aupetit, S., \& Oliviero, H. (2008). Etude de la dynamique d'apprentissage et des habiletés à la conduite "deux roues" motorisés en situation réelle : concevoir des dispositifs de formation intégrant des simulateurs. Communication orale présentée lors de la biennale de l'ARIS. Rodez, 14-16 Mai 2008.

Ayers, S., \& Hawkins, A. (2002). Evaluation des savoirs conceptuels sous-disciplinaires en éducation physique. Communication affichée présentée lors de la biennale de I'ARIS. Rennes, 12 -14 Décembre 2002.

Aymé, S., Ferrand, C., \& Cogérino, G. (2005). Les transactions et les émotions des enseignants experts d'éducation physique et sportive. Communication orale présentée lors de la biennale de l'ARIS. Louvain-La-Neuve, 20 -22 Janvier 2005.

Azaïs, S., Cizeron, M., \& Gal, N. (2002). Le temps vécu par l'enseignant d'EPS stagiaire en situation d'enseignement. Communication orale présentée lors de la biennale de l'ARIS. Rennes, 12 -14 Décembre 2002.

Baba-Moussa, Rahamane, A., \& Garncarzick, C. (2006). Les liens entre théorie et pratique dans la formation des enseignants d'EPS : comment les étudiants perçoivent-ils I'utilité de savoirs théoriques ? Communication affichée présentée lors de la biennale de l'ARIS. Besançon, 9-12 Mai 2006.

Baeza, N. et Bouthier, D. (2006). Observer et décrire les " zones de rencontre " en EPS : lieu d'interaction et de transmission des savoirs. Communication orale présentée lors de la biennale de l'ARIS. Besançon, 9-12 Mai 2006.

Ballot, G., Musard, M., \& Grandclément, P. (2008). L'Education à l'Environnement pour un Développement Durable (EEDD) en Education Physique et Sportive. Communication orale présentée lors de la biennale de l'ARIS. Rodez, 14-16 Mai 2008.

Barbot, A., \& Saujat, F. (2000). "Instruction au sosie" et développement des compétences professionnelles : le cas d'une PCL2 EPS. Communication affichée présentée lors de la biennale de l'ARIS. Grenoble, 14-16 Décembre 2000.

Baron, F., \& Loquet, M. (2002). Réflexion méthodologique initiale dans le cadre d'une recherche didactique : de l'intérêt d'explorer l'ordinaire. Communication affichée présentée lors de la biennale de l'ARIS. Rennes, 12 -14 Décembre 2002.

Barthès, D. (2006). L'évaluation vidéographique en EPS ou comment innover dans l'évaluation des productions motrices en EPS. Communication affichée présentée lors de la biennale de l'ARIS. Besançon, 9-12 Mai 2006. 
Baudet, P., \& Carnel, B. (2005). Evaluation certificative et référentiel pour le baccalauréat en course pédestre de durée : C.C.5. Communication orale présentée lors de la biennale de l'ARIS. Louvain-La-Neuve, 20 -22 Janvier 2005.

Beaudoin, C., Leblanc, R., \& Lauzon, P. (2005). L'effet des cours d'éducation physique sur la performance académique d'élèves franco-ontariens, en dixième année du secondaire. Communication affichée présentée lors de la biennale de I'ARIS. Louvain-La-Neuve, 20 -22 Janvier 2005.

Beaudoin, C., Rivard, M-C., Grenier, J., \& Gréhaigne, J.F. (2006). Construire sa santé à l'école : l'apport des éps. Communication affichée présentée lors de la biennale de I'ARIS. Besançon, 9-12 Mai 2006.

Beaudoin, S., Harnois, H., \& Brunelle, JP. (2008). Le développement de la responsabilité : une recherche-action en EP au primaire. Communication orale présentée lors de la biennale de l'ARIS. Rodez, 14-16 Mai 2008.

Beaulieu, J., Rivard, M-C., \& Castani, M. (2008). Point de vue des acteurs eu égard aux facteurs favorables et obstacles liés au processus de la triade. Communication orale présentée lors de la biennale de l'ARIS. Rodez, 14-16 Mai 2008.

Beltramini, L. Delfosse, C., Piéron, M., \& Cloes, M. (2000). Regards sur les jeunes participant à des activités sportives organisées dans des quartiers défavorisés. Communication affichée présentée lors de la biennale de l'ARIS. Grenoble, 14-16 Décembre 2000.

Ben Jomaa, H., \& Terrisse, A. (2008). Le rapport au savoir de l'enseignant d'EPS. Approche clinique et implications didactiques. Communication orale présentée lors de la biennale de l'ARIS. Rodez, 14-16 Mai 2008.

Bennour, N. (2008). L'évolution des planifications pré et post actives des étudiants stagiaires d'EPS. Identification des compétences didactiques durant le " stage de préparation à la vie professionnelle ". Communication orale présentée lors de la biennale de l'ARIS. Rodez, 14-16 Mai 2008.

Berchebru, M., \& Poggi, MP. (2006). Relation EPS et santé : quels rapports avec l'obésité ? Communication affichée présentée lors de la biennale de l'ARIS. Besançon, 912 Mai 2006.

Bergé, 2006 Apprendre le ressenti dans les activités scolaires d'entretien et Estime de Soi. Communication affichée présentée lors de la biennale de l'ARIS. Besançon, 9-12 Mai 2006. 
Bernard, D., Trudel, P., \& Marcotte, G. (2000). Les possibilités d'éducation morale en hockey sur glace. Communication affichée présentée lors de la biennale de I'ARIS. Grenoble, 14-16 Décembre 2000.

Bertone, S. (2002). Dynamique des interactions en situation de conseil pédagogique et développement de l'activité professionnelle d'une enseignante novice d'éducation physique et son conseiller pédagogique. Communication orale présentée lors de la biennale de l'ARIS. Rennes, 12 -14 Décembre 2002.

Bertone, S., Euzet, JP., Méard, JA., \& Flavier, E. (2000). Les conflits intrapsychiques d'un enseignant novice d'EPS dans ses interactions avec des élèves de collège : étude de cas. Communication affichée présentée lors de la biennale de l'ARIS. Grenoble, 14-16 Décembre 2000.

Bizzonni-Previeux, C., Grenier, J., Merini, C., Jourdan, D., \& Otis, J. (2008). Éducateurs physiques et à la santé à l'école primaire : travail individuel ou collectif ? Communication orale présentée lors de la biennale de l'ARIS. Rodez, 14-16 Mai 2008.

Blocher, JN., \& Durny, A. (2002). Que deviennent les plans dans l'action? Illustration en volley-ball pour des élèves de classe de Terminale en EPS. Communication affichée présentée lors de la biennale de l'ARIS. Rennes, 12 -14 Décembre 2002.

Blocher, JN., \& Durny, A. (2005). L'élève : un acteur sous contraintes. Communication affichée présentée lors de la biennale de l'ARIS. Louvain-La-Neuve, $20-22$ Janvier 2005.

Bolatre, A., \& Carnel, B. (2005). La formation des agrégatifs en Education Physique et Sportive à l'épreuve orale vidéo. Communication affichée présentée lors de la biennale de l'ARIS. Louvain-La-Neuve, 20 -22 Janvier 2005.

Bonnard, A., \& Wallian, N. (2006). Stratégies interprétatives et lecture du parcours en course d'orientation : l'évaluation des élèves en classe de Terminale au Baccalauréat. Communication affichée présentée lors de la biennale de l'ARIS. Besançon, 9-12 Mai 2006.

Bordes, P. (2005). Intervention et dynamique de groupe. Formes de regroupements et performances des élèves en Éducation Physique. Communication affichée présentée lors de la biennale de l'ARIS. Louvain-La-Neuve, 20 -22 Janvier 2005.

Borges, C., \& Rondea, S. (2008). Travail curriculaire et pratique enseignante en éducation physique dans le contexte du Renouveau pédagogique au Québec. Communication orale présentée lors de la biennale de l'ARIS. Rodez, 14-16 Mai 2008. 
Borges, C., \& Rondeau, S. (2008). La collaboration entre pairs : les défis que doivent surmonter les éducateurs physiques pour briser leur solitude. Communication orale présentée lors de la biennale de l'ARIS. Besançon, 9-12 Mai 2006.

Borges, C, Kalinova, E., \& Le Cren, F. (2006). L'éducation à la santé : Un défi pour les enseignants en éducation physique au Québec. Communication orale présentée lors de la biennale de l'ARIS. Besançon, 9-12 Mai 2006.

Bosseray, A., \& Avanzini, G. (2000). Analyse de l'activité compétitive d'un golfeur : étude de cas. Communication affichée présentée lors de la biennale de l'ARIS. Grenoble, 14-16 Décembre 2000.

Bothelo-Gomes, P., Graça, A., Siva, P., \& Queiros, P. (2005). Opportunités de pratique sportive selon le sexe perçues par des élèves de l'enseignement secondaire. Communication affichée présentée lors de la biennale de l'ARIS. Louvain-LaNeuve, 20 -22 Janvier 2005.

Boudard, JM. (2006). Les régulations didactiques en Education Physique et Sportive : un geste professionnel. Communication affichée présentée lors de la biennale de l'ARIS. Besançon, 9-12 Mai 2006.

Boudreau, P. (2005). La réussite en éducation physique : les perspectives d'adolescents, d'enseignants en formation et d'enseignants d'expérience. Communication affichée présentée lors de la biennale de l'ARIS. Louvain-La-Neuve, 20 -22 Janvier 2005.

Bouslama, F. (2008). Profil du Jeune Tunisien physiquement éduqué tel qu'il est souhaité par les enseignants d'Education Physique. Communication affichée présentée lors de la biennale de l'ARIS. Besançon, 9-12 Mai 2006.

Brau-Antony, S., \& David, B. (2000). Le référentiel d'évaluation au baccalauréat comme révélateur des conceptions des enseignants d'éducation physique et sportive. Communication affichée présentée lors de la biennale de l'ARIS. Grenoble, 14-16 Décembre 2000.

Brau-Antony, S. (2006). L'observation didactique au service du développement de compétences professionnelles de formateurs d'enseignants. Communication orale présentée lors de la biennale de l'ARIS. Besançon, 9-12 Mai 2006.

Breton, C., Mahut, N., \& Gréhaigne, JF. (2002). Gestuelle communicationnelle dans l'intervention en EPS : microanalyse d'un cas en gymnastique sportive. Communication affichée présentée lors de la biennale de l'ARIS. Grenoble, 14-16 Décembre 2000. 
Brière-Guenoun, F., \& Amade-Escot, C. (2005). De la conception des dispositifs d'apprentissage à l'étude des connaissances de l'enseignant : le cas d'un enseignant novice dans une situation de franchissement au saut de cheval. Communication orale présentée lors de la biennale de l'ARIS. Louvain-La-Neuve, 20 -22 Janvier 2005.

Briot, M. (2000). Application des nouveaux programmes dans les associations sportives scolaires. Rôle de l'action organisée. Communication affichée présentée lors de la biennale de l'ARIS. Grenoble, 14-16 Décembre 2000.

Briot, M. (2002). Justifications et stratégies curriculaires en EPS. Communication orale présentée lors de la biennale de l'ARIS. Rennes, 12 -14 Décembre 2002.

Brunelle, JP., Martel, D., Gagnon, D., Tousignant, M., Goyette, R., Brunelle, J., \& Spallanzani, C. (2000). La connaissance des iniquités perçues par les élèves dans les cours d'éducation physique au primaire : une entrée prometteuse en supervision de la qualité de l'intervention pédagogique. Communication affichée présentée lors de la biennale de l'ARIS. Grenoble, 14-16 Décembre 2000.

Bui-Xuân, G., Carlier, B., \& Bouthier, D. (2005). Intervenir en éducation physique et en sport. Recherches actuelles. Louvain : Presses Universitaires de Louvain.

Camire, M., \& Trudel, P. (2008). La pratique sportive en milieu scolaire : la perspective des élèves-athlètes. Communication orale présentée lors de la biennale de l'ARIS. Rodez, 14-16 Mai 2008.

Carlier, G., \& Vandesande, I. (2002). Maître de stage, c'est-à-dire? Ou comment des maîtres de stage définissent leur fonction. Communication orale présentée lors de la biennale de l'ARIS. Rennes, 12 -14 Décembre 2002.

Carlier, G., Daele, JM., \& Chaumont, E. (2008). Analyse de l'accompagnement de maîtres de stage par des superviseurs universitaires. Communication orale présentée lors de la biennale de l'ARIS. Rodez, 14-16 Mai 2008.

Carlier, G., Faber, C., \& Petesch, C. (2008). La notion de plaisir en Education Physique (et Sportive) : Études de cas en France, en Belgique et au Grand-duché de Luxembourg. Communication affichée présentée lors de la biennale de l'ARIS. Rodez, 14-16 Mai 2008.

Carnel, B., \& Delhemmes, R. (2000). EPS : Changement des textes, changement des pratiques ? Etude de pratiques de stagiaires en situation, en collège. Communication affichée présentée lors de la biennale de l'ARIS. Grenoble, 14-16 Décembre 2000. 
Carnel, B., Masschelein, V., \& Boutoille, O. (2008). Combat et Escalade au CAS de Roubaix. Communication orale présentée lors de la biennale de l'ARIS. Rodez, 14-16 Mai 2008.

Carnus, M.F. (2002). Esquisse de modélisation du processus décisionnel de l'enseignant d'EPS - Une approche didactique. Communication orale présentée lors de la biennale de l'ARIS. Rennes, 12 -14 Décembre 2002.

Carpentier, S., \& Mercier Lefèvre, B. (2006). L'interaction entraîneur entraîné dans le discours des judokas : Une modélisation socio-anthropologique à partir de l'échange don. Communication affichée présentée lors de la biennale de l'ARIS. Besançon, 9-12 Mai 2006.

Carton, A. (2005). Variabilité des stratégies de coping chez des experts et des débutants au cours de différentes pratiques sportives. Communication affichée présentée lors de la biennale de l'ARIS. Louvain-La-Neuve, 20 -22 Janvier 2005.

Caty, D., \& Gréhaigne, JF. (2000). L'évaluation en Acrosport : conception et validation d'un outil. Communication affichée présentée lors de la biennale de l'ARIS. Grenoble, 14-16 Décembre 2000.

Caty, D., \& Musard, M. (2006). Des techniques acrobatiques pour dynamiser l'acrosport scolaire. Communication affichée présentée lors de la biennale de l'ARIS. Besançon, 9-12 Mai 2006.

Chaliès, S., \& Escalie, G. (2008). Faire vivre le collectif de travail en formation : Postulats théoriques et grandes lignes. Communication orale présentée lors de la biennale de l'ARIS. Rodez, 14-16 Mai 2008.

Chaliès, S., \& Flavier, E. (2002). Le stage pratique : un véritable lieu de formation des enseignants stagiaires. Communication affichée présentée lors de la biennale de l'ARIS. Rennes, 12 -14 Décembre 2002.

Chaliès, S. (2000). Analyse du cours d'action de tuteurs lors des séances conduites par des enseignants en formation : une observation participante. Communication affichée présentée lors de la biennale de l'ARIS. Grenoble, 14-16 Décembre 2000.

Chaliès, S. Trohel, J., \& Durand, M. (2000). Articulation des cours d'action des enseignants stagiaires et de leurs conseillers pédagogiques lors d'entretiens postleçon. Communication affichée présentée lors de la biennale de l'ARIS. Grenoble, 14-16 Décembre 2000.

Chaliès, S., Flavier, E., \& Bertone, S. (2006). Vers une rénovation de la situation traditionnelle de conseil pédagogique. Communication orale présentée lors de la biennale de l'ARIS. Besançon, 9-12 Mai 2006. 
Chang, CW., Mahut, N., \& Gréhaigne, JF. (2002). Cultures professionnelles et résistances au changement des enseignants du primaire. Communication orale présentée lors de la biennale de l'ARIS. Rennes, 12 -14 Décembre 2002.

Chang, CW., Wallian, N., \& Gréhaigne, JF. (2008). La réception du courant "Teaching Games for Understanding " (TGfU) à Taiwan : approche de la formation continue des maîtres du Primaire et de Collège. Communication orale présentée lors de la biennale de l'ARIS. Rodez, 14-16 Mai 2008.

Chang, CW., Wallian, N., \& Nachon, M. (2006). Débat d'idées et interlocutions discursives en basket-ball : les rapports de position dans les stratégies d'opposition. Communication orale présentée lors de la biennale de l'ARIS. Besançon, 9-12 Mai 2006.

Charest, R., Roy, M., Perreault, G., \& Brunelle, JP. (2008). Formation à l'entraînement à la prise de décisions en tennis par une démarche d'autosupervision. Communication affichée présentée lors de la biennale de l'ARIS. Rodez, 14-16 Mai 2008.

Château, L., Gréhaigne, JF., \& Caty, D. (2005). Modélisation du ballon prisonnier : impact d'une phase de verbalisation. Communication affichée présentée lors de la biennale de l'ARIS. Louvain-La-Neuve, 20 -22 Janvier 2005.

Chaumont, E., Carlier, G., \& Dervaux, P. (2005). La course de longue durée à l'école : ce qu'en disent des enseignants. Communication orale présentée lors de la biennale de l'ARIS. Louvain-La-Neuve, 20 -22 Janvier 2005.

Chigot, D., Grison, B., \& Riff, J. (2000). Danser le Rock'N'Roll en EPS au collège : une affaire de réseau - L'exploitation de ressources "distribuées" dans la construction d'une activité de danseur en couple. Communication affichée présentée lors de la biennale de l'ARIS. Grenoble, 14-16 Décembre 2000.

Cicero, C., \& Lafont, L. (2005). Comparaison de deux modalités d'imitation-modélisation interactive au sein d'interactions de tutelle entre élèves. L'effet de la formation des tuteurs en gymnastique sportive. Communication orale présentée lors de la biennale de l'ARIS. Louvain-La-Neuve, 20 -22 Janvier 2005.

Cizeron, M., \& Gal, N. (2002). Archétype et connaissance pragmatique des contenus matière chez les enseignants d'EPS. Communication orale présentée lors de la biennale de l'ARIS. Rennes, 12 -14 Décembre 2002.

Cizeron, M., \& Gal-Petitfaux, N. (2005). Le travail en " vagues " et en " ateliers " : deux façons d'enseigner et d'apprendre au cours de leçons de gymnastique. Communication orale présentée lors de la biennale de l'ARIS. Louvain-La-Neuve, 20 -22 Janvier 2005. 
Cizeron, M., \& Gal-Petitfaux, N. (2006). Du désarroi professionnel au remaniement de la conception de l'APS : Etude de cas d'un dispositif de formation par la démarche réflexive. Communication orale présentée lors de la biennale de l'ARIS. Besançon, 9-12 Mai 2006.

Cizeron, M. (2006). Médiation dans la transmission des techniques du corps : la question des croyances délirantes. Communication orale présentée lors de la biennale de l'ARIS. Besançon, 9-12 Mai 2006.

Cizeron, M., Gal, N., \& Clace, AS. (2008). La métaphore de l'énergie dans trois contextes d'intervention. Communication orale présentée lors de la biennale de l'ARIS. Rodez, 14-16 Mai 2008.

Clerc, C., Wallian, N., Musard, M., \& Poggi, MP. (2005). Lecture interprétative des conduites motrices et expertise professionnelle. Communication orale présentée lors de la biennale de l'ARIS. Louvain-La-Neuve, 20 -22 Janvier 2005.

Cleuziou, JP., \& Pontais, C. (2006). L'enseignement de l'EPS et les professeurs des écoles. Communication orale présentée lors de la biennale de l'ARIS. Besançon, 9-12 Mai 2006.

Cleuziou, JP. (2000). Approche technologique en didactique des APS et recherche sur la construction de tables de cotation. Communication orale présentée lors de la biennale de l'ARIS. Grenoble, 14-16 Décembre 2000.

Cleuziou, JP., Brau-Antony, S., \& David, B. (2002). Aspects de l'évaluation en éducation physique et sportive au baccalauréat français. Communication orale présentée lors de la biennale de l'ARIS. Rennes, 12 -14 Décembre 2002.

Cloes, M., Bonfond, JM., \& Lenzen, B. (2002). Analyse des démarches cognitives associées à l'émission d'informations collectives. Communication affichée présentée lors de la biennale de l'ARIS. Rennes, 12 -14 Décembre 2002.

Cloes, M., Lenzen, B., Sikora, I., \& Pieron, M. (2000). Prise de décisions liées à la gestion d'une équipe de basket-ball en situation de compétition. Etude de cas. Communication affichée présentée lors de la biennale de l'ARIS. Grenoble, 14-16 Décembre 2000.

Cloes, M., Maraite, AA., Zhang, L., Albert, A., \& Theunissen, C. (2008). Perceptions du style de vie chez des élèves de l'enseignement primaire. Effets d'un programme expérimental visant à doubler le nombre de leçons d'éducation physique. Communication orale présentée lors de la biennale de l'ARIS. Rodez, 14-16 Mai 2008. 
Cloes, M., Résimont, B., \& Piéron, M. (2000). Analyse des interventions verbales dont les joueurs se souviennent à l'issue d'une rencontre de sport collectif. Communication affichée présentée lors de la biennale de l'ARIS. Grenoble, 14-16 Décembre 2000.

Clot, Y. (2003). Le collectif dans I'individu ?. In G. Vallery et R. Amalberti (Eds.), Modèles et pratiques de l'analyse du travail. 1988-2003, 15 ans d'évolution. Actes du XXXVIIle Congrès de la SELF. Paris : SELF.

Cogérino, G., \& Lounana, J. (2006). Interagir avec les élèves : catégorisation et mémorisation des élèves chez les enseignants d'EPS. Communication orale présentée lors de la biennale de l'ARIS. Besançon, 9-12 Mai 2006.

Cogérino, G., \& Mnafakh, H. (2008). Conceptions de l'équité chez les enseignants d'EPS et pratiques évaluatives. Communication orale présentée lors de la biennale de l'ARIS. Besançon, 9-12 Mai 2006.

Cogérino, G. (2002). Impact différencié d'une séquence d'enseignement selon le sexe et le genre de l'élève. Communication affichée présentée lors de la biennale de I'ARIS. Rennes, 12 -14 Décembre 2002.

Cogérino, G., Perrin, C., \& Motta, D. (2000). Impact sur les pratiques professionnelles et conceptions de l'enseignement de l'EP de la participation à une recherche : étude de cas en Education à la Santé. Communication affichée présentée lors de la biennale de I'ARIS. Grenoble, 14-16 Décembre 2000.

Cogérino, G., Terret, T., \& Rogowski, I. (2005). Mise en place de la mixité en EPS et expertise professionnelle. Communication orale présentée lors de la biennale de l'ARIS. Louvain-La-Neuve, 20 -22 Janvier 2005.

Coignus, G., Famose, JP., \& Margnes, E. (2008). Fixation et révision de but en EPS en réponse à un décalage but/performance. Communication affichée présentée lors de la biennale de l'ARIS. Besançon, 9-12 Mai 2006.

Coltice, M., \& Cogérino, G. (2002). Pratiques physiques et artistiques et construction identitaire de genre. Communication affichée présentée lors de la biennale de l'ARIS. Rennes, 12 -14 Décembre 2002.

Coltice, M. (2000). L'enseignement de la danse au collège : Le "pratiquant culture". Un outil d'analyse. Communication affichée présentée lors de la biennale de l'ARIS. Grenoble, 14-16 Décembre 2000.

Coltice, M., Couchot-Schiex, S.,, \& Cogérino, G. (2006). Les enseignants stagiaires sontils préoccupés par la mixité ? Communication orale présentée lors de la biennale de l'ARIS. Besançon, 9-12 Mai 2006. 
Cordoba, A., Dénervaud, H., Deriaz, D., Lenzen, B., \& Poussin, B. (2008). Étude comparative des conceptions d'étudiants en formation dans différentes filières à propos de l'enseignement des jeux sportifs collectifs. Communication affichée présentée lors de la biennale de l'ARIS. Rodez, 14-16 Mai 2008.

Corneloup, J. (2000). Processus d'ancrage au métier de guide de montagne. Communication affichée présentée lors de la biennale de l'ARIS. Grenoble, 14-16 Décembre 2000.

Costes, L., \& Amade-Escot, C. (2005). Effets de l'intervention différenciée de l'enseignant sur les dynamiques d'apprentissage des filles et des garçons dans l'activité football. Communication orale présentée lors de la biennale de l'ARIS. LouvainLa-Neuve, 20 -22 Janvier 2005.

Costes, L., \& Amade-Escot, C. (2005). Effets de l'intervention différenciée de l'enseignant sur les dynamiques d'apprentissage des filles et des garçons dans l'activité football. Communication orale présentée lors de la biennale de l'ARIS. LouvainLa-Neuve, 20 -22 Janvier 2005.

Costes, L., \& Deutz d'Arragon, S. (2002). Les différences de traitement des filles et des garçons dans les interactions pédagogiques en EPS. Communication affichée présentée lors de la biennale de l'ARIS. Rennes, 12 -14 Décembre 2002.

Couchot-Schiex, S. (2006). " L'univers " de l'enseignant d'EPS est-il genre ? Communication orale présentée lors de la biennale de l'ARIS. Besançon, 9-12 Mai 2006.

Couchot-Schiex, S., Trottin, B., \& Cogérino, G. (2008). Apprendre en mixité : un casse-tête fantasmé ou un support pour les apprentissages ? Etude auprès d'enseignants stagiaires en EPS. Effets de différenciation sexuée des interactions en EPS. Communication orale présentée lors de la biennale de l'ARIS. Rodez, 14-16 Mai 2008.

Couderc, B., \& Léziart, Y. (2002). Influence des repères perceptifs sur les facteurs du mouvement dans l'improvisation en danse. Communication orale présentée lors de la biennale de l'ARIS. Rennes, 12 -14 Décembre 2002.

Cousin, S., Brunelle, JP., Dugas, C., \& Spallanzani, C. (2006). Effet d'un programme individualisé en éducation physique qui vise le développement de l'équilibre et de la coordination chez des élèves de 8 et 9 ans. Communication affichée présentée lors de la biennale de l'ARIS. Besançon, 9-12 Mai 2006.

Crispim-Santos, A., \& Rodrigues, J. (2006). Analyse de l'instruction de l'entraîneur de football. Comparaison entre les instructions de préparation et la compétition. 
Communication affichée présentée lors de la biennale de l'ARIS. Besançon, 9-12 Mai 2006.

Daele, JM., Carlier, G., \& Dervaux, P. (2005). La leçon d'éducation physique : Comment des professeurs stagiaires la préparent-ils ? Communication orale présentée lors de la biennale de l'ARIS. Louvain-La-Neuve, 20 -22 Janvier 2005.

Darnis, F. (2008). Analyse interlocutoire du conseil en formation : conseil ou formation ? Communication orale présentée lors de la biennale de l'ARIS. Rodez, 14-16 Mai 2008.

Darnis, L., Lafont, L., \& Menaut, A. (2006). Interactions verbales en situation de coconstruction de règles d'action au handball : L'exemple de deux dyades à fonctionnement contrasté. Communication orale présentée lors de la biennale de I'ARIS. Besançon, 9-12 Mai 2006.

David, B., Amarouche, P., \& Choffin, T. (2000). La confrontation de l'expérience comme instrument de formation à l'évaluation des professeurs stagiaires d'EPS. Communication affichée présentée lors de la biennale de l'ARIS. Grenoble, 14-16 Décembre 2000.

De Fabrique, V., \& Cogérino, G. (2006). Rapport au corps et à l'apparence, modèles de masculinité/féminité dans les activités d'entretien. Communication orale présentée lors de la biennale de l'ARIS. Besançon, 9-12 Mai 2006.

Debars, C., \& Amade-Escot, C. (2005). Enseigner le badminton à une classe de primoarrivants : incidence du contexte ZEP sur la pratique d'intervention. Communication orale présentée lors de la biennale de l'ARIS. Louvain-La-Neuve, 20 -22 Janvier 2005.

Dechef, C., \& Cogérino, G. (2005). Les enseignants face à l'écart de notation entre les filles et les garçons au baccalauréat. Communication affichée présentée lors de la biennale de l'ARIS. Louvain-La-Neuve, 20 -22 Janvier 2005.

Deglau, D., \& O'Sullivan, M. (2005). Influence de l'intervention en formation professionnelle continue sur les croyances et les pratiques d'enseignants expérimentés des "Urban Schools". Communication orale présentée lors de la biennale de l'ARIS. Louvain-La-Neuve, 20 -22 Janvier 2005.

Delrieu, B., Mouchet, A., \& Bouthier, D. (2008). La gestion des remplacements de joueurs en match par l'entraîneur professionnel de rugby. Communication orale présentée lors de la biennale de l'ARIS. Rodez, 14-16 Mai 2008. 
Demers, G. (2002). Analyse de besoins en sport des athlètes et des entraîneurs de haut niveau. Communication affichée présentée lors de la biennale de l'ARIS. Rennes, 12 -14 Décembre 2002.

Deriaz, D., \& Poussin, B. (2000). Influence d'un contexte de rénovation sur les conceptions d'intervention des enseignants : une étude à propos de l'enseignement en basket-ball. Communication affichée présentée lors de la biennale de l'ARIS. Grenoble, 14-16 Décembre 2000.

Derome, S., Pirson, S., \& Cloes, M. (2005). Analyse de la préparation de match chez un entraîneur de football expérimenté. Communication affichée présentée lors de la biennale de l'ARIS. Louvain-La-Neuve, 20 -22 Janvier 2005.

Dervaux, P., Carlier, G., \& Gérard, JP. (2005). Regards croisés des stagiaires et des élèves sur l'origine d'incidents disciplinaires significatifs au cours d'Education physique. Communication orale présentée lors de la biennale de l'ARIS. LouvainLa-Neuve, 20 -22 Janvier 2005.

Desbiens, JF. (2002). Contrôle et régulation de la supervision active en enseignement de l'éducation physique au primaire. Communication orale présentée lors de la biennale de l'ARIS. Rennes, 12 -14 Décembre 2002.

Desbiens, JF., Roy, M., Spallanzani, C., Brunelle, JP., Turcotte, S., \& Lanoue, S. (2005). Le sentiment d'efficacité à gérer la classe de stagiaires en enseignement de l'ÉPS. Communication orale présentée lors de la biennale de I'ARIS. Louvain-La-Neuve, 20 -22 Janvier 2005.

Desbiens, JF., Roy, M., Spallanzani, C., Brunelle, JP., Turcotte, S., \& Lanoue, S. (2005). Le sentiment d'efficacité à gérer la classe de stagiaires en enseignement de l'ÉPS. Communication orale présentée lors de la biennale de l'ARIS. Louvain-La-Neuve, 20 -22 Janvier 2005.

Desbiens, JF., Spallanzani, C., \& Tourigny, JS. (2008). Un exemple d'initiative originale mis en place par des enseignants d'éducation physique et à la santé d'une école secondaire en vue d'améliorer la qualité de l'encadrement de leurs élèves. Communication affichée présentée lors de la biennale de l'ARIS. Rodez, 14-16 Mai 2008.

Desbiens, JF., Turcotte, S., Spallanzani, C., Bergevin, N., Brière, I., Tourigny, K., \& Tourigny, JS. (2006). Comment des stagiaires en enseignement de l'éducation physique et à la santé utilisent-ils le questionnement auprès des élèves? Communication affichée présentée lors de la biennale de l'ARIS. Besançon, 9-12 Mai 2006. 
Desigaux, F., Sève, C., \& Adé, D. (2005). Engagement, attentes et émotions chez une enseignante d'EPS stagiaire. Communication affichée présentée lors de la biennale de l'ARIS. Louvain-La-Neuve, 20 -22 Janvier 2005.

Devos-Prieur, O., \& Amade-Escot, C. (2005). Influence du profil d'action des Professeurs sur les contenus enseignés en Education Physique : étude croisée de quatre professeurs des écoles. Communication orale présentée lors de la biennale de l'ARIS. Louvain-La-Neuve, 20 -22 Janvier 2005.

Devos-Prieur, O., \& Amans-Passaga, C. (2008). Professeur des écoles et intervenant extérieur en EPS : nature de la collaboration et incidence sur le choix des savoirs mis à l'étude. Communication orale présentée lors de la biennale de l'ARIS. Rodez, 14-16 Mai 2008.

Donzé, B., \& Durand, M. (2000). L'entraînement des grimpeurs experts : émotion et coopération au service de la conception des circuits en situation d'entraînement. Communication affichée présentée lors de la biennale de l'ARIS. Grenoble, 14-16 Décembre 2000.

Dubé, F., \& Delens, C. (2000). Motricité et adaptation scolaire - Le regard de l'enseignant : pré tests du dispositif expérimental. Communication affichée présentée lors de la biennale de I'ARIS. Grenoble, 14-16 Décembre 2000.

Dugal, JP. (2005). Savoirs didactiques et construction de savoirs professionnels chez des enseignants conseillers pédagogiques. Communication orale présentée lors de la biennale de l'ARIS. Louvain-La-Neuve, 20 -22 Janvier 2005.

Dupont, JP., Collignon, C., \& Carlier, G. (2008). Le plaisir d'enseigner l'éducation physique dans l'enseignement secondaire spécialisé de forme 3 . Communication affichée présentée lors de la biennale de l'ARIS. Rodez, 14-16 Mai 2008.

Dupont, JP., \& Garcia, A. (2005). La pratique sportive régulière et les études universitaires. Communication affichée présentée lors de la biennale de l'ARIS. Louvain-La-Neuve, 20 -22 Janvier 2005.

Dupont, JP., Gérard, P., Delens, C., \& Carlier, G. (2008). Les négociations "professeur élèves" et leurs relations avec les processus motivationnels des élèves en éducation physique : une approche basée sur la théorie de l'auto-détermination. Communication orale présentée lors de la biennale de l'ARIS. Besançon, 9-12 Mai 2006.

Duprat, E., \& Gréhaigne, JF. (2006). Relations entre le " barycentre " de la récupération du ballon et la performance. Communication affichée présentée lors de la biennale de l'ARIS. Besançon, 9-12 Mai 2006. 
Duprat, E., \& Trimoreau, E. (2008). Approche technologique sur les différents types de pertes du ballon. Communication orale présentée lors de la biennale de l'ARIS. Rodez, 14-16 Mai 2008.

Durny, A. (2006). La séance d'évaluation : un format d'organisation pédagogique complexe. Communication affichée présentée lors de la biennale de l'ARIS. Besançon, 9-12 Mai 2006.

Durny, A., Coulomb, G., Melet, P., Pasco, D., Petit, E., Guibal, S, \& Le Bastard, F. (2000). Enseignement de l'EPS et maîtrise de l'activité support par l'enseignant : une étude de cas. Communication affichée présentée lors de la biennale de l'ARIS. Grenoble, 14-16 Décembre 2000.

Dusson, P., \& Marchal, D. (2002). La distinction complexité/difficulté dans les tâches de sauts athlétiques. Implication sur l'étude des stratégies d'apprentissage mises en jeu par les élèves. Communication affichée présentée lors de la biennale de l'ARIS. Rennes, 12 -14 Décembre 2002.

Dusson, P., Riff, J., \& David, B. (2006). L'identité composite de joueurs de handball et action située : étude de la dynamique identitaire dans une perspective de conception ergonomique. Communication orale présentée lors de la biennale de I'ARIS. Besançon, 9-12 Mai 2006.

Dusson, P., Riff, J., \& David, B. (2008). L'engagement au sein d'une structure de haut niveau : la notion de responsabilité dans le développement à l'adolescence. Communication orale présentée lors de la biennale de l'ARIS. Rodez, 14-16 Mai 2008.

El Andoulsi, S., \& Bedhioufi, H. (2008). Etude comparative des deux enseignants d'éducation physique d'expertises différentes dans l'enseignement de l'appui tendu renversé. Communication orale présentée lors de la biennale de l'ARIS. Rodez, 14-16 Mai 2008.

El Andoulsi, S., Bedhioufi, H., \& Amade-Escot, C. (2006). Analyse didactique de l'intervention de l'enseignant d'EPS en gymnastique: les processus différentiels selon le sexe et le niveau d'habileté des élèves dans l'enseignement de l'ATR. Communication affichée présentée lors de la biennale de l'ARIS. Besançon, 9-12 Mai 2006.

El Andoulsi, S., Bedhioufi, H., \& Amade-Escot, C. (2006). Analyse didactique de l'intervention de l'enseignant d'EPS en gymnastique: les processus différentiels selon le sexe et le niveau d'habileté des élèves dans l'enseignement de l'ATR. 
Communication affichée présentée lors de la biennale de l'ARIS. Besançon, 9-12 Mai 2006.

Eloi, S. (2002). Identification des compétences des passeurs de haut niveau en volley-ball. Communication orale présentée lors de la biennale de l'ARIS. Rennes, 12 -14 Décembre 2002.

Eloi, S. (2008). Identification du style de 5 passeurs de haut niveau en volley-ball. Communication orale présentée lors de la biennale de l'ARIS. Rodez, 14-16 Mai 2008.

Ensergueix, P., \& Lafont, L. (2006). Tutorat réciproque entre élèves : étude des conditions favorables au managérat d'un pair en tennis de table. Communication orale présentée lors de la biennale de l'ARIS. Besançon, 9-12 Mai 2006.

Euzet, JP., Méard, A., \& Bertone, S., \& Gal, N. (2000). Le conseil pédagogique dans la formation professionnelle des enseignants d'éducation physique : étude de cas. Communication affichée présentée lors de la biennale de l'ARIS. Grenoble, 14-16 Décembre 2000.

Fache, H., \& Recopé, M. (2006). Sentir, se mouvoir et perception du monde : l'enjeu de rupture de l'échange comme sensibilité partagée. Communication affichée présentée lors de la biennale de l'ARIS. Besançon, 9-12 Mai 2006.

Falga, N., \& Monnier, N. (2008). L'activité didactique en milieu difficile : intérêt d'un collectif de recherche élargi. Communication orale présentée lors de la biennale de l'ARIS. Rodez, 14-16 Mai 2008.

Ferrand , C., \& Helvig, MM. (2006). La gymnastique rythmique et la mixité : quels regards portent des étudiant(e)s sur cette activité ? Communication orale présentée lors de la biennale de l'ARIS. Besançon, 9-12 Mai 2006.

Fischback, W., \& Cloes, M. (2005). Analyse de la mise en pratique d'un module de formation continuée en jonglerie par des enseignants du secondaire. Communication orale présentée lors de la biennale de l'ARIS. Louvain-La-Neuve, 20 -22 Janvier 2005.

Flavier, E., \& Durand, M. (2002). Les conflits professeur(s)-élèves : des situations profitables et formatrices? Communication orale présentée lors de la biennale de I'ARIS. Rennes, 12 -14 Décembre 2002.

Flavier, E., \& Chaliès, C. (2006). Développement de l'activité professionnelle d'un enseignant novice exerçant en zone d'éducation prioritaire. Communication orale présentée lors de la biennale de l'ARIS. Besançon, 9-12 Mai 2006. 
Flavier, E., Bertone, S., \& Méard, A. (2000). L'organisation typique des conflits professeur - élève(s) lors de leçons d'EPS en collège. Communication affichée présentée lors de la biennale de l'ARIS. Grenoble, 14-16 Décembre 2000.

Flavier, E., Bertone, S., Chaliès, C., \& Méard, J. (2006). Impact de la formation sur le développement de l'activité professionnelle des enseignants novices (EN). Communication affichée présentée lors de la biennale de l'ARIS. Besançon, 9-12 Mai 2006.

Franco, S., Rodrigues, J., \& Castaner, M. (2006). Comportement pédagogique des instructeurs de fitness. Communication affichée présentée lors de la biennale de l'ARIS. Besançon, 9-12 Mai 2006.

Frédéric, O., \& Cloes, M. (2008). Comparaison des stratégies d'enseignement du basketball en milieu scolaire et en milieu sportif. Communication affichée présentée lors de la biennale de l'ARIS. Rodez, 14-16 Mai 2008.

Fréderic, O., Delhaes, D., \& Cloes, M. (2006). Comparaison des représentations de l'approche didactique du basket-ball en milieu scolaire et en milieu sportif. Communication affichée présentée lors de la biennale de l'ARIS. Besançon, 9-12 Mai 2006.

Frédéric, O., Gribomont, J., \& Cloes, M. (2008). Identification de stratégies d'enseignement du basket-ball en milieu sportif. Communication orale présentée lors de la biennale de l'ARIS. Rodez, 14-16 Mai 2008.

Frédéric, O., Louis, L., \& Cloes, M. (2005). Impact d'un sport-études sur la vie d'un établissement d'enseignement secondaire. Communication affichée présentée lors de la biennale de l'ARIS. Louvain-La-Neuve, 20 -22 Janvier 2005.

Gagné, G., Martel, D., \& Nadeau, L. (2005). Révélations d'élèves du secondaire sur la mixité en éducation physique. Communication affichée présentée lors de la biennale de l'ARIS. Louvain-La-Neuve, 20 -22 Janvier 2005.

Gagnon, J., Normandin, JM., Nadeau, L., Martel, D., \& Michaud, V. (2006). Stratégies d'élèves pour réaliser avec succès un programme de stimulation à la pratique d'activité physique. Communication orale présentée lors de la biennale de l'ARIS. Besançon, 9-12 Mai 2006.

Gagnon, J., Martel, D., Michaud, V., Valois, P., \& Gagné, G. (2002). Analyse des types de concordance-discordance entre ce que des élèves pensent d'eux-mêmes et ce qu'ils croient que leur éducateur physique pense d'eux. Communication orale présentée lors de la biennale de l'ARIS. Rennes, 12 -14 Décembre 2002. 
Gagnon, J., Nadeau, L., Martel, D., \& Normandin, JP. (2006). Le pentathlon en équipe : un programme efficace pour inciter les jeunes à la pratique d'activité physique. Communication orale présentée lors de la biennale de l'ARIS. Besançon, 9-12 Mai 2006.

Gal, N., \& Cizeron, M. (2005). Motifs " cachés " et motifs " apparents " de la donation de parole aux élèves en classe : Eude de cas d'enseignants débutants en Education physique. Communication orale présentée lors de la biennale de l'ARIS. LouvainLa-Neuve, 20 - 22 Janvier 2005.

Gal, N., \& Cizeron, M. (2006). Intelligibilité de la pratique EPS et construction des savoirs d'expérience par l'analyse réflexive : étude de cas d'un stagiaire-plc2. Communication orale présentée lors de la biennale de l'ARIS. Besançon, 9-12 Mai 2006.

Gal, N., \& Ria, L. (2002). La formation par l'action des enseignants débutants en éducation physique et sportive : la construction de nouvelles connaissances en classe. Communication orale présentée lors de la biennale de l'ARIS. Rennes, 12 -14 Décembre 2002.

Gal-Petitfaux, N. (2006). Les injonctions dans la communication en classe : des énoncés simples masquant une compétence discursive chez l'enseignant d'EPS. Communication orale présentée lors de la biennale de l'ARIS. Besançon, 9-12 Mai 2006.

Gantcheva, G., Mineva, M., Loquet, M., \& Léziart, Y. (2008). Optimiser l'entraînement en gymnastique rythmique, illustration à travers l'enseignement et l'apprentissage des sauts. Communication affichée présentée lors de la biennale de l'ARIS. Rodez, 14-16 Mai 2008.

Garnier, A., \& Goirand, P. (2000). Le basket ball, une pratique chargée de sens... oui mais lequel ? Communication affichée présentée lors de la biennale de l'ARIS. Grenoble, 14-16 Décembre 2000.

Garnier, A. (2005). Les gestes du professeur d'EPS lors de l'étude de l'appui tendu renversé, au collège. Communication orale présentée lors de la biennale de I'ARIS. Louvain-La-Neuve, 20 -22 Janvier 2005.

Garnier, A. (2006). Du contrat de communication au contrat didactique : le jeu des phénomènes interactifs dans la transmission du savoir. Communication orale présentée lors de la biennale de l'ARIS. Besançon, 9-12 Mai 2006. 
Gautron, M., \& Cogérino, G. (2002). Le rôle de l'expérience vécue dans la construction de l'identité de futurs professeurs d'EPS. Communication orale présentée lors de la biennale de l'ARIS. Rennes, 12 -14 Décembre 2002.

Generolo, E., Zaragoza, J., Julian, JA., \& Lopez, J. (2006). CALADU : un programme informatique pour la gestion des cours de course de durée. Communication affichée présentée lors de la biennale de l'ARIS. Besançon, 9-12 Mai 2006.

Gérard, JP. (2002). Modélisation du développement de la foulée de l'enfant entre 2 et 11 ans. Communication affichée présentée lors de la biennale de l'ARIS. Rennes, 12 -14 Décembre 2002.

Gojard, L., \& Terral, P. (2008). De l'étudiant STAPS à l'enseignant d'EPS : une "désportivisation" nécessaire. Communication orale présentée lors de la biennale de l'ARIS. Rodez, 14-16 Mai 2008.

Gouju, JL. (2002). Vers des outils d'aide à l'intervention en courses de haies hautes. Communication orale présentée lors de la biennale de l'ARIS. Rennes, 12 -14 Décembre 2002.

Gouju, JL. (2005). L'œil du maquignon comme compétence professionnelle des enseignants d'Education Physique et Sportive. Communication affichée présentée lors de la biennale de l'ARIS. Louvain-La-Neuve, 20 -22 Janvier 2005.

Graça, A., Bothelo-Gomes, P., Silva, P., \& Queiros, P. (2005). Croyances des étudiants de Sport et Education Physique à propos des objectifs et des valeurs de l' Education Physique. Communication affichée présentée lors de la biennale de l'ARIS. Louvain-La-Neuve, 20 -22 Janvier 2005.

Grandclément, P., \& Musard, M. (2008). La dimension artistique dans le système enseignement-apprentissage en EPS. Communication orale présentée lors de la biennale de l'ARIS. Rodez, 14-16 Mai 2008.

Grassetie, P., \& Bouthier, D. (2006). Conceptions et gestions des situations de référence dans l'enseignement des jeux et sports collectifs au cycle 2 de l'école primaire : incidences pour la formation des professeurs des écoles en EPS. Communication orale présentée lors de la biennale de l'ARIS. Besançon, 9-12 Mai 2006.

Gréhaigne, JF., Caty, D., \& Château, L. (2005). Modélisations de l'attaque et didactique des sports collectifs en EPS. Communication orale présentée lors de la biennale de I'ARIS. Louvain-La-Neuve, 20 -22 Janvier 2005.

Grenier, J., \& Kalinova, E. (2005). L'évaluation des élèves en éducation physique. Le point de vue des enseignants en formation. Communication affichée présentée lors de la biennale de I'ARIS. Louvain-La-Neuve, 20 -22 Janvier 2005. 
Grenier, J., Boudreau, P., Richard, JF., Raiche, G., Boudreault, I., \& Raymond, J. (2008). L'évaluation des apprentissages en éducation physique peut-elle être authentique ? Communication orale présentée lors de la biennale de l'ARIS. Rodez, 14-16 Mai 2008.

Grenier, J., Raymond, J., Bégin, J., Lauzon, A., Verret, N., \& Gagné, J. (2006). Attentes et perceptions d'élèves, de parents et d'éducateurs physiques en regard des pratiques évaluatives en éducation physique au primaire. Communication orale présentée lors de la biennale de l'ARIS. Besançon, 9-12 Mai 2006.

Guérin, J. (2006). Composantes publique et clandestine de l'activité d'élèves en tennis de table. Communication orale présentée lors de la biennale de l'ARIS. Besançon, 912 Mai 2006.

Guillou, J., \& Durny, A. (2000). Etude d'une situation d'enseignement en EPS : Les points de vue l'enseignant et de l'élève. Communication affichée présentée lors de la biennale de I'ARIS. Grenoble, 14-16 Décembre 2000.

Guillou, J., \& Durny, A. (2006). Analyse sémiologique de l'activité de l'élève en éducation physique : la gestion du duel en badminton. Communication orale présentée lors de la biennale de l'ARIS. Besançon, 9-12 Mai 2006.

Hadjb Taieb, P., \& Hentati, A. (2008). L'éducation psychomotrice à l'école primaire en Tunisie, entre rationalité politique et réalité pratique. Communication orale présentée lors de la biennale de l'ARIS. Rodez, 14-16 Mai 2008.

Haise, A. (2000). Le processus d'incorporation en kayak : contribution à une approche d'anthropologie cognitive aux problèmes de l'intervention. Communication affichée présentée lors de la biennale de l'ARIS. Grenoble, 14-16 Décembre 2000.

Haye, G. (2005). Le plaisir : un processus de liaison entre intervenants et pratiquants dans les APS. Communication affichée présentée lors de la biennale de l'ARIS. Louvain-La-Neuve, 20 -22 Janvier 2005.

Huet, B., \& Saury, J. (2008). Dynamique des interactions, construction et partage de connaissances en cours d'EPS. Communication orale présentée lors de la biennale de l'ARIS. Rodez, 14-16 Mai 2008.

Huet, B., Saury, J., \& Rossard, C. (2006). Fluctuations des préoccupations de collégiens au cours de leçons d'éducation physique en relation avec les différentes situations de classe. Communication orale présentée lors de la biennale de l'ARIS. Besançon, 9-12 Mai 2006. 
Huillerey, A., \& Keimbou, DC. (2000). Structure des interactions en classe de sixième : statuts sociométriques et réseaux de communication. Communication affichée présentée lors de la biennale de l'ARIS. Grenoble, 14-16 Décembre 2000.

Hutteau, F., \& Avanzini, G. (2000). Analyse d'une collaboration entraîneur-athlète non prescriptive : étude de cas en canoë. Communication affichée présentée lors de la biennale de l'ARIS. Grenoble, 14-16 Décembre 2000.

Imbert (2006). La transmission des savoirs dans la formation d'enseignants entre utilitarisme et cycles de don: illustration en éducation physique. Communication orale présentée lors de la biennale de l'ARIS. Besançon, 9-12 Mai 2006.

Kammoun, M., \& Amade-Escot, C. (2005). La gestualité de l'enseignant d'EPS : Modalités et fonctionnalités didactiques selon l'expertise gymnique. Communication orale présentée lors de la biennale de l'ARIS. Louvain-La-Neuve, 20 -22 Janvier 2005.

Kermarrec, G.,\& Léziart, Y. (2002). Les stratégies d'autorégulation en EPS - Une étude descriptive de l'activité des élèves en contexte scolaire. Communication orale présentée lors de la biennale de l'ARIS. Rennes, 12 -14 Décembre 2002.

Kermoal, C., \& Gréhaigne, JF. (2006). Saut en hauteur scolaire : espaces subjectifs d'action dans la construction motrice personnelle au cours de l'apprentissage de sauts de type fosbury flop. Communication affichée présentée lors de la biennale de l'ARIS. Besançon, 9-12 Mai 2006.

Kervella, Y., \& Léziart, Y. (2002). Technologie du football, essai de caractérisation des techniques de frappes de balle des joueurs experts - L'exemple des passes d'élimination. Communication orale présentée lors de la biennale de l'ARIS. Rennes, 12 -14 Décembre 2002.

Kirk, D., \& Kirk, S. (2008). Coopération chercheur/enseignants : A la découverte des apports éducationnels d'un programme "Sport Education" en éducation physique à l'école primaire. Communication orale présentée lors de la biennale de l'ARIS. Rodez, 14-16 Mai 2008.

Kirk, D., MacPhail, A., \& Griffin, L (2005). Progressions d'apprentissage d'écolier de 10 ans dans un cycle de jeux d'invasion. Communication orale présentée lors de la biennale de l'ARIS. Louvain-La-Neuve, 20 -22 Janvier 2005.

Kohler, M., \& Gréhaigne, JF. (2000). La reconnaissance visuelle d'une habileté gymnique : comparaison d'enseignants experts et polyvalents. Communication affichée présentée lors de la biennale de l'ARIS. Grenoble, 14-16 Décembre 2000.

Kohler, M., Stucchi, N., Papaxanthis, C., \& Pozzo, T. (2002). L'apprentissage par observation : effets des informations visuelles statiques et/ou dynamiques sur 
l'amélioration de la performance physique en gymnastique sportive. Communication affichée présentée lors de la biennale de l'ARIS. Rennes, 12 -14 Décembre 2002.

Kozanitis, A., \& Desbiens, JF. (2005) L'environnement d'apprentissage et son influence sur les interactions verbales des étudiants de premier cycle universitaire en ÉPS. Communication orale présentée lors de la biennale de l'ARIS. Louvain-La-Neuve, 20 -22 Janvier 2005.

Krause, S., \& Saury, J. (2002). De l'analyse de l'activité experte en funboard à la conception d'aides à l'entraînement. Communication orale présentée lors de la biennale de l'ARIS. Rennes, 12 -14 Décembre 2002.

Lab, F., Marchal, D., \& Gréhaigne, JF. (2002). La régularité d'allure constitue-t-elle un objectif pertinent en course de durée? Communication affichée présentée lors de la biennale de l'ARIS. Rennes, 12 -14 Décembre 2002.

Labrude, N., Musard, M., \& Wallian, N. (2006). Genèse d'une chorégraphie en danse contemporaine. Communication affichée présentée lors de la biennale de I'ARIS. Besançon, 9-12 Mai 2006.

Lacroix, AL., Gal-Petiffaux, N., \& Cizeron, M. (2002). L'activité d'enseignants d'EPS expérimentés en situation de début de leçon. Communication orale présentée lors de la biennale de l'ARIS. Rennes, 12 -14 Décembre 2002.

Lafont, L., \& Proeres, M. (2000). Mettre en place des discussions entre pairs à propos d'un jeu sportif collectif, Quelle analyse des interactions ? Communication affichée présentée lors de la biennale de l'ARIS. Grenoble, 14-16 Décembre 2000.

Lafont, L. (2005). Apports de la psychologie sociale à l'intervention en EPS : rôle des interactions tutorielles et des " coping " modèles. Communication orale présentée lors de la biennale de l'ARIS. Louvain-La-Neuve, 20 -22 Janvier 2005.

Lafont, L., Rubert, O., \& Vincent-Morin, M. (2008). EPS et santé en Lycées et Lycées Professionnels aquitains : quelles procédures de guidage ? Rôles des APS supports et du contexte scolaire. Communication orale présentée lors de la biennale de l'ARIS. Rodez, 14-16 Mai 2008.

Laraki, N., Ancion, G., \& Cloes, M. (2005). Fidéliser sa clientèle ? Un exemple de démarche dans le milieu du fitness. Communication affichée présentée lors de la biennale de l'ARIS. Louvain-La-Neuve, 20 -22 Janvier 2005.

Launay, E., \& Avanzini, G. (2000). Etude du pilotage de karting : fonctionnement intrinsèque et perspective d'intervention. Communication affichée présentée lors de la biennale de l'ARIS. Grenoble, 14-16 Décembre 2000. 
Le Bas, A. (2000). La continuité des apprentissages dans les activités gymniques, au cycle des apprentissages fondamentaux de l'école primaire. L'exemple du saut de mouton. Communication affichée présentée lors de la biennale de l'ARIS. Grenoble, 14-16 Décembre 2000.

Le Bas, A. (2002). Identification de quelques déterminants de l'action de l'enseignant du premier degré débutant, en EPS. Communication affichée présentée lors de la biennale de I'ARIS. Rennes, 12 -14 Décembre 2002.

Le Pallec, A., \& Léziart, Y. (2002). Le jeu de rupture/continuité chez les jeunes joueurs de tennis (8-14 ans). Communication orale présentée lors de la biennale de l'ARIS. Rennes, 12 -14 Décembre 2002.

Le Paven, M. (2008). Les savoirs intermédiaires : un concept utile pour caractériser la relation didactique entraîneur / athlète. Communication affichée présentée lors de la biennale de l'ARIS. Rodez, 14-16 Mai 2008.

Leblanc, S. (2000). Autoformation dans un environnement d'apprentissage multimédia conçu comme une aide pour " Penser l'entraînement sportif ". Communication affichée présentée lors de la biennale de l'ARIS. Grenoble, 14-16 Décembre 2000.

Lebouvier, B., \& Ouitre, F. (2002). Transformation de la relation à l'effort chez des lycéens en course longue. Communication affichée présentée lors de la biennale de l'ARIS. Rennes, 12 -14 Décembre 2002.

Lebouvier, B. (2006). Cadres interprétatifs mobilisés par le conseiller pédagogique pour analyser des situations d'enseignement - apprentissage en EPS. Communication orale présentée lors de la biennale de l'ARIS. Besançon, 9-12 Mai 2006.

Lecomte, J., Carlier, G., \& Renard, JP. (2000). Analyse du réinvestissement auprès de leurs élèves d'expériences d'apprentissage réalisées par des enseignants en éducation physique dans un contexte de formation continue. Communication affichée présentée lors de la biennale de l'ARIS. Grenoble, 14-16 Décembre 2000.

Lecoq, JC. (2008). Un nouvel outil pour l'entraîneur, Q.P.M Sport. Communication orale présentée lors de la biennale de l'ARIS. Rodez, 14-16 Mai 2008.

Lecuivre, G. (2006). Empêchements et développement du pouvoir d'agir. Communication affichée présentée lors de la biennale de l'ARIS. Besançon, 9-12 Mai 2006.

Lefèvre, F., Habsch, I., \& Cloes, M. (2005). Analyse de la problématique de l'abandon en natation de competition. Communication affichée présentée lors de la biennale de l'ARIS. Louvain-La-Neuve, 20 -22 Janvier 2005.

Legrand, P., \& Refuggi, R. (2006). Étude exploratoire de la gestualité enseignante et de ses fonctionnalités didactiques : le cas de deux professeurs d'EPS de spécialité 
sportive enseignée différente. Communication affichée présentée lors de la biennale de l'ARIS. Besançon, 9-12 Mai 2006.

Lemoine, A. (2006). Les configurations typiques du jeu en déviations en football : reconnaissance et apprentissage. Communication orale présentée lors de la biennale de l'ARIS. Besançon, 9-12 Mai 2006.

Lémonie, Y., David, B., \& Gouju, J.L. (2006). Les interactions didactiques comme construction d'un espace partage. Le cas de l'enseignement de la natation sportive. Communication orale présentée lors de la biennale de l'ARIS. Besançon, 9-12 Mai 2006.

Lemonie, Y., Robin, JF., Le Gall, B., \& David, B. (2008). Gestes professionnels et dimension interactive du métier d'enseignant : une analyse comparative dans trois disciplines. Communication orale présentée lors de la biennale de l'ARIS. Rodez, 14-16 Mai 2008.

Lemyre, F., Trudel, P., \& Culver, D. (2008). L'utilisation d'Internet par des entraîneurs canadiens. Communication affichée présentée lors de la biennale de l'ARIS. Rodez, 14-16 Mai 2008.

Lemyre, F., Trudel, P., \& Durand-Bush, N. (2006). Le parcours d'apprentissage d'entraîneurs bénévoles dans trois disciplines sportives. Communication affichée présentée lors de la biennale de l'ARIS. Besançon, 9-12 Mai 2006.

Lentillon, V., \& Cogérino, G. (2002). La perception par les élèves des inégalités entre les sexes dans l'évaluation en EPS. Communication affichée présentée lors de la biennale de l'ARIS. Rennes, 12 -14 Décembre 2002.

Lentillon, V., \& Cogérino, G. (2005). L'efficacité du soutien d'enseignants plus ou moins expérimentés : points de vue d'élèves du second degré. Communication orale présentée lors de la biennale de l'ARIS. Louvain-La-Neuve, 20 -22 Janvier 2005.

Lenzen, B., Delfosse, C., Menten, R., Piéron, M., \& Cloes, M. (2000). Analyse des rituels d'athlètes belges francophones en période pré-compétitive. Communication affichée présentée lors de la biennale de l'ARIS. Grenoble, 14-16 Décembre 2000.

Lenzen, B., Denervaud, H., Poussin, B., Renggli, P., \& Dubbach, A. (2008). " Imaginez que vous êtes le formateur de terrain de cet étudiant stagiaire. " Préparation virtuelle d'un entretien post-séance par les pairs. Communication orale présentée lors de la biennale de l'ARIS. Rodez, 14-16 Mai 2008.

Lenzen, B., Gustin, F., \& Cloes, M. (2005). Evaluation de l'efficacité d'un module d'enseignant à distance centré sur l'analyse de tâche et l'émission de feedback. 
Communication orale présentée lors de la biennale de l'ARIS. Louvain-La-Neuve, 20 -22 Janvier 2005.

Lenzen, B., Theunissen, C., \& Cloes, M. (2006). Prise de décision en handball : points de vue interne des joueuses et externe du coach. Communication affichée présentée lors de la biennale de l'ARIS. Besançon, 9-12 Mai 2006.

Leriche, J., Desbiens, JF., \& Dugal, JP. (2008). L'accompagnement des stagiaires : un enjeu pour la formation des enseignants. Communication orale présentée lors de la biennale de l'ARIS. Rodez, 14-16 Mai 2008.

Letort, O. (2000). La compétition amateur adultes en tennis : proposition de formats de jeu adaptés au tennis évolutif. L'exemple du Tennis Cooleurs. Communication affichée présentée lors de la biennale de l'ARIS. Grenoble, 14-16 Décembre 2000.

Léziart, Y., \& Pasco, D. (2006). Les programmes en éducation physique et sportive et leur mise en pratique dans les établissements scolaires. Communication orale présentée lors de la biennale de l'ARIS. Besançon, 9-12 Mai 2006.

Loiseau, S., Brunelle, JP., Lemieux, G., Roy, M., \& Turcotte, S. (2006). L'impact des occasions de répondre dans un processus d'intégration de l'entraînement à la prise de décision. Communication affichée présentée lors de la biennale de I'ARIS. Besançon, 9-12 Mai 2006.

Loizon, D., \& Martin, D. (2005). Des savoirs enseignés à l'expérience des enseignants. Terrain et objet de la collaboration. Communication orale présentée lors de la biennale de l'ARIS. Louvain-La-Neuve, 20 -22 Janvier 2005.

Loizon, D., \& Terrisse, A. (2000). Les professeurs de judo et la transmission du savoir. Communication affichée présentée lors de la biennale de l'ARIS. Grenoble, 14-16 Décembre 2000.

Loizon, D., \& Terrisse, A. (2002). Analyse des pratiques d'enseignement du judo en club. Communication affichée présentée lors de la biennale de l'ARIS. Rennes, 12 -14 Décembre 2002.

Longchamp, L., \& Cogérino, G. (2006). Buts poursuivis et perception des obstacles a la pratique en EPS : le cas des jeunes filles musulmanes. Communication affichée présentée lors de la biennale de l'ARIS. Besançon, 9-12 Mai 2006.

Loquet, M., \& Gantcheva, G. (2006). La production des savoirs dans l'entraînement de haute performance : Etude comparée en France et en Bulgarie de l'action effective des entraîneurs en gymnastique rythmique (GR). Communication orale présentée lors de la biennale de l'ARIS. Besançon, 9-12 Mai 2006. 
Loquet, M. (2000). Elaborer un contenu d'enseignement en EPS: apports de l'ingénierie didactique. Etude de cas en gymnastique rythmique. Communication orale présentée lors de la biennale de l'ARIS. Grenoble, 14-16 Décembre 2000.

Loquet, M., \& Léziart, Y. (2004). Cultures sportives et artistiques. Formalisation des savoirs professionnels. Rennes : PUHB et ARIS. .

Loriot, M., \& Avanzini, G. (2000). Conception d'un système d'aide : le cas d'une relation coach-athlète " problématique " en escrime. Communication affichée présentée lors de la biennale de l'ARIS. Grenoble, 14-16 Décembre 2000.

Lorrain, A., \& Froissart, T. (2006). Tableaux organisateurs de la motricité pendulaire et acrobatique: des outils au service de l'enseignement des activités gymniques et acrobatiques. Communication orale présentée lors de la biennale de l'ARIS. Besançon, 9-12 Mai 2006.

Louis, E., \& Refuggi, R. (2002). Pilotage tactique du jeu par un joueur de tennis de niveau intermédiaire en situation de match. Communication orale présentée lors de la biennale de l'ARIS. Rennes, 12 -14 Décembre 2002.

Louis, E., \& Refuggi, R. (2006). La technologie des aps au sein du cursus staps : " vers un état des lieux ". Communication orale présentée lors de la biennale de I'ARIS. Besançon, 9-12 Mai 2006.

Louis, E., Refuggi, R., \& Attali, M. (2005). Technologie : Réflexions et travaux du syndicat national de I'EPS. Communication orale présentée lors de la biennale de I'ARIS. Louvain-La-Neuve, 20 -22 Janvier 2005.

Machemehl, C., \& Lefèvre, JP. (2006). Contribution a une histoire de l'éducation physique et sportive entre 1945 et 1967 : d'un modèle d'enseignement imposé vers une action régulée. Communication affichée présentée lors de la biennale de I'ARIS. Besançon, 9-12 Mai 2006.

Magendie, E. (2008) Comprendre l'activité des élèves en EPS à partir des décalages entre le prévu et le réalisé. Communication orale présentée lors de la biennale de I'ARIS. Rodez, 14-16 Mai 2008.

Mahut, B., Mahut, N., \& Gréhaigne, JF. (2000). La gestuelle communicative dans la professionnalité enseignante : le cas de l'EPS. Communication affichée présentée lors de la biennale de l'ARIS. Grenoble, 14-16 Décembre 2000.

Mandy Destailleur, A., \& Poggi, MP. (2006). Construction scolaire d'une APSA : le cas du cirque. Communication affichée présentée lors de la biennale de l'ARIS. Besançon, 9-12 Mai 2006. 
Manteau, T., \& Testevuide, S. (2005). Analyse située de l'improvisation collective en danse. Communication affichée présentée lors de la biennale de l'ARIS. LouvainLa-Neuve, 20 -22 Janvier 2005.

Marchal, D., Delachau, N., \& Lab, F. (2006). La réalité de l'escalade enseignée en milieu scolaire. Communication affichée présentée lors de la biennale de l'ARIS. Besançon, 9-12 Mai 2006.

Marre, S. (2008). Théories d'action didactique des formateurs en sport collectif et projet de formation à l'intervention en licence STAPS. Communication orale présentée lors de la biennale de l'ARIS. Rodez, 14-16 Mai 2008.

Marsault, C. (2000). Evolution des conceptions de l'EPS : Influence des modes de recrutements des professeurs d'EPS sur la définition de leur discipline. Communication affichée présentée lors de la biennale de l'ARIS. Grenoble, 14-16 Décembre 2000.

Martel, D., Gagnon, J., \& Demers, G. (2002). Anatomie d'une recherche-action. Communication orale présentée lors de la biennale de l'ARIS. Rennes, 12 -14 Décembre 2002.

Martel, D., Gagnon, J., Brunelle, JP., Tousignant, M., Goyette, R., Brunelle, J., \& Spallanzani, C. (2000). Réactions d'éducateurs physiques à des situations que leurs élèves considèrent injustes en éducation physique. Communication affichée présentée lors de la biennale de l'ARIS. Grenoble, 14-16 Décembre 2000.

Martel, D., Gagnon, J., \& Godbout, P. (2008). Des réalités diverses vécues par des élèves qui s'estiment évalués à leur juste valeur par leur éducateur physique.

Martin, L., \& Lafont, L. (2005). " Coping model " et Modèle de maîtrise : l'influence du type de modélisation sur l'apprentissage d'une habileté gymnique et sur les réponses psychologiques des sujets. Communication orale présentée lors de la biennale de l'ARIS. Louvain-La-Neuve, 20 -22 Janvier 2005.

Mascret, N. (2008). Les interactions "joueur - coach" en badminton et leur impact sur les apprentissages en EPS des élèves difficiles. Communication orale présentée lors de la biennale de I'ARIS. Besançon, 9-12 Mai 2006.

Massey, M., \& Grenier, J. (2008). Vers la validation d'un test de mesure du niveau de collaboration en co-enseignement de l'éducation physique et à la santé. Communication affichée présentée lors de la biennale de l'ARIS. Rodez, 14-16 Mai 2008. 
Masson, P., Carnel, B., \& Garnier, A. (2005). Corps, conflit(s) et espace : Quelle(s) prise(s) en compte en Education Physique ? Communication affichée présentée lors de la biennale de l'ARIS. Louvain-La-Neuve, 20 -22 Janvier 2005.

Mauffrey, R., \& Poggi, MP. (2006). Influences du sexe de l'enseignant et du type d' APSA (historiquement masculine et féminine) sur les différences de contenus d'enseignement donnés aux filles et aux garçons. Communication affichée présentée lors de la biennale de l'ARIS. Besançon, 9-12 Mai 2006.

Méard, JA. Bertone, S., Euzet, JP., \& Ria, L. (2000). La présentation du travail scolaire et son effet sur l'activité des élèves. Comparaison de deux professeurs d'éducation physique en leçon de badminton en collège.

Mees, V., Carlier, G., \& Renard, JP. (2000). Diriger un établissement scolaire : quelles préoccupations vis-à-vis de l'éducation physique ? Communication affichée présentée lors de la biennale de l'ARIS. Grenoble, 14-16 Décembre 2000.

Michaud, V., Gauthier, K., Gagnon, J., \& Demers, G. (2005). Stratégies d'éducation à la santé qui visent à rendre les élèves plus actifs. Communication affichée présentée lors de la biennale de l'ARIS. Louvain-La-Neuve, 20 -22 Janvier 2005.

Mikulovic, J., Bui-Xuan, G., \& Theunynck, D. (2005). Du débutant à l'expérimenté : l'intervenant en APS auprès de populations en difficultés d'adaptation sociale dans la gestion du conflit socio-conatif. Communication orale présentée lors de la biennale de l'ARIS. Louvain-La-Neuve, 20 -22 Janvier 2005.

Mokhenache, A., Wallian, N., \& Gréhaigne, JF. (2005). L'UFR STAPS de Besançon et l'intervention. Communication orale présentée lors de la biennale de l'ARIS. Louvain-La-Neuve, 20 -22 Janvier 2005.

Mokhenache, A., Wallian, N., \& Gréhaigne, JF. (2005). L'UFR-STAPS de Besançon et l'intervention. Communication affichée présentée lors de la biennale de l'ARIS. Louvain-La-Neuve, 20 -22 Janvier 2005.

Moniotte, J., \& Poggi, MP. (2006). EPS et rapport(s) au corps. Communication affichée présentée lors de la biennale de l'ARIS. Besançon, 9-12 Mai 2006.

Montaud, D. (2008). De l'observation des pratiques au rapport au savoir de l'enseignant dans l'interaction didactique : le cas de deux hommes enseignant la danse. Communication orale présentée lors de la biennale de l'ARIS. Rodez, 14-16 Mai 2008.

Montreuil, R., Lacroix, C., \& Trudel, P. (2006). Le sport scolaire au canada : la perception des entraîneurs du rôle des directeurs d'écoles. Communication affichée présentée lors de la biennale de l'ARIS. Besançon, 9-12 Mai 2006. 
Morency, L., Bordeleau, C., \& Léone, M. (2008). La recherche collaborative : une stratégie de recherche prometteuse pour réduire l'écart entre la culture du chercheur et celle du praticien. Communication affichée présentée lors de la biennale de l'ARIS. Rodez, 14-16 Mai 2008.

Moreno, G. (2006). Formes de mixité a l'école et inégalités de réussite entre filles et garçons. Communication orale présentée lors de la biennale de l'ARIS. Besançon, 9-12 Mai 2006.

Mouchet, A. (2006). La co-construction du référentiel commun en rugby. Communication affichée présentée lors de la biennale de l'ARIS. Besançon, 9-12 Mai 2006.

Mouchet, A. (2008). Le coaching des sélections nationales en rugby : observation et analyse du jeu en début de match. Communication orale présentée lors de la biennale de l'ARIS. Rodez, 14-16 Mai 2008.

Moulin, N., Lafont, L., \& Menaut, A. (2005). L'entraîneur confronté au syndrome de burnout des athlètes : cas du tennis de haut niveau. Communication affichée présentée lors de la biennale de l'ARIS. Louvain-La-Neuve, 20 -22 Janvier 2005.

Muguet, JP., Léziart, Y., \& Carnel, B. (2008). L'influence des contextes sur la réalisation d'actions particulières de tirs en basket ball. Communication orale présentée lors de la biennale de l'ARIS. Rodez, 14-16 Mai 2008.

Munoz, L., \& Mierjewski, S. (2005). Le corps et l'expérience au service de l'enseignement supérieur, L'exemple des STAPS. Communication orale présentée lors de la biennale de l'ARIS. Louvain-La-Neuve, 20 -22 Janvier 2005.

Musard, M., \& Léziart, Y. (2000). La construction des savoirs par un groupe d'élèves de $5^{\circ}$ en acrosport. Communication affichée présentée lors de la biennale de l'ARIS. Grenoble, 14-16 Décembre 2000.

Musard, M., Robin, JF., Mahut, N., \& Gréhaigne, JF. (2002). Composition "sous influences" des curricula et diversité des pratiques scolaires : le cas de l'acrosport. Communication orale présentée lors de la biennale de l'ARIS. Rennes, 12 -14 Décembre 2002.

Musard, M., Thieblemont, M., \& Caty, D. (2005). Le système enseignement-apprentissage au cours d'un cycle acrosport en classe de troisième. Communication orale présentée lors de la biennale de l'ARIS. Louvain-La-Neuve, 20 -22 Janvier 2005.

Nachon, M., Wallian, N., \& Gréhaigne, JF. (2005). Enseignant et élèves en situation d'interlocutions au basket-ball : Etude de cas sur les processus de construction des savoirs. Communication orale présentée lors de la biennale de l'ARIS. Louvain-La-Neuve, 20 -22 Janvier 2005. 
Nadeau, L., \& Poirier, B. (2006). La mesure de la stabilité de la mesure en situation réelle de jeu au hockey sur glace. Communication affichée présentée lors de la biennale de l'ARIS. Besançon, 9-12 Mai 2006.

Nunes, WJ., Beltrao, FB., Dos Santos, CM., \& Boga Filho, HS. (2006). Analyse du degré de satisfaction des élèves d'éducation physique de l'université fédérale et rurale de Rio de Janeiro dans les cours de tennis sur gazon. Communication affichée présentée lors de la biennale de l'ARIS. Besançon, 9-12 Mai 2006.

O'Sullivan, M. (2005). Programmes de formation professionnelle continue qui assoient le pouvoir d'action des enseignants d'éducation physique. Communication orale présentée lors de la biennale de l'ARIS. Louvain-La-Neuve, 20 -22 Janvier 2005.

Oudot, E., \& Poggi, MP. (2008). L'activité réflexive des élèves en demi-fond : stratégies développées et rapport au savoir. Communication orale présentée lors de la biennale de l'ARIS. Besançon, 9-12 Mai 2006.

Ouitre, F. (2006). Caractérisation du profil professionnel de stagiaire PLC 2 en éducation physique et sportive et transformation en cours de formation de ce profil. Communication orale présentée lors de la biennale de l'ARIS. Besançon, 9-12 Mai 2006.

Outrey, E., Mahut, N., \& Gréhaigne, JF. (2000). L'observation des élèves en natation scolaire par l'association de mots. Communication orale présentée lors de la biennale de l'ARIS. Louvain-La-Neuve, 20 -22 Janvier 2005.

Patinet, C., \& Cogérino, G. (2008). Centration attentionnelle des enseignants d'EPS en contexte mixte. Communication orale présentée lors de la biennale de l'ARIS. Rodez, 14-16 Mai 2008.

Patinet, C. (2005). Pratiques pédagogiques relatives à la mixité en Education Physique et Sportive : mises en œuvre et analyses réflexives des enseignants de collège. Communication affichée présentée lors de la biennale de l'ARIS. Louvain-LaNeuve, 20 -22 Janvier 2005.

Pehoiu, C. (2006). L'optimisation de l'activité d'éducation physique des préadolescents a base des contenus éducationnels à effets complexes d'instruction et de formation spécifiques à l'athlétisme. Communication affichée présentée lors de la biennale de l'ARIS. Besançon, 9-12 Mai 2006.

Pehoiu, C., Savu, C., \& Chesnoiu, L. (2008). Les jeux de mouvement, méthode et moyens efficients de formation des capacités coordinatives pendant les classes d'éducation physique des préadolescents. Communication orale présentée lors de la biennale de l'ARIS. Rodez, 14-16 Mai 2008. 
Perez-Roux, T. (2008). L'agir professionnel des enseignants-stagiaires d'EPS : autour d'une séance de danse en classe de cinquième mixte. Communication orale présentée lors de la biennale de l'ARIS. Rodez, 14-16 Mai 2008.

Perreault, G., Roy, M., Desbiens, JF., \& Spallanani, C. (2006). Description du profil comportemental d'ordre communicationnel d'un entraîneur de volley-ball expert au cours d'une saison sportive. Communication affichée présentée lors de la biennale de l'ARIS. Besançon, 9-12 Mai 2006.

Perreault, G., Roy, M., Desbiens, JF., \& Harnois, H. (2006). Exploration du raisonnement sous-jacent à la communication d'un entraîneur expert en volley-ball au cours d'une saison sportive. Communication affichée présentée lors de la biennale de I'ARIS. Besançon, 9-12 Mai 2006.

Perrin, C., \& Lefèbvre, (2006). Co-construction de contenus interdisciplinaires d'éducation pour la santé : Perspective d'une sociologie pragmatique.

Peter, JM. (2006). Construction de savoir à travers les récits de 42 joueurs de tennis experts. Communication affichée présentée lors de la biennale de l'ARIS. Besançon, 9-12 Mai 2006.

Petit, E., Durny, A., Coulomb, G., Melet, F., Pasco, D., Guibald, S., \& Le Bastard, F. (2000). Interactions Enseignant - Elèves en classe de 6ème : une étude de cas. Communication affichée présentée lors de la biennale de l'ARIS. Grenoble, 14-16 Décembre 2000.

Poggi-Combaz, MP., \& Mahut, B. (2002). Les activités de pleine nature en milieu scolaire Discours sur les pratiques et discours sur les principes : des distorsions. Communication orale présentée lors de la biennale de I'ARIS. Rennes, 12 -14 Décembre 2002.

Poggi-Combaz, MP. (2002). L'apport de la sociologie du curriculum aux travaux sur les curricula en EPS. Communication orale présentée lors de la biennale de I'ARIS. Rennes, 12 -14 Décembre 2002.

Poizat, G., Adé, G., Gal-Petitgaux, N., \& Seifert, L. (2008). Analyse des interactions entre des nageurs de haut niveau et un instrument de mesure subaquatique. Communication orale présentée lors de la biennale de l'ARIS. Rodez, 14-16 Mai 2008.

Pontais, C. (2000). Contribution à la formation des enseignants à partir de documents didactiques. L'exemple de la gymnastique rythmique (GR) à l'école primaire. Communication affichée présentée lors de la biennale de l'ARIS. Grenoble, 14-16 Décembre 2000. 
Poulin, N., \& Avanzini, G. (2000). L'analyse de l'activité d'un joueur de tennis en compétition. Présentation de l'analyse au joueur comme système d'aide. Communication affichée présentée lors de la biennale de l'ARIS. Grenoble, 14-16 Décembre 2000.

Serres, G., Ria, L., Adé, D., , \& Sève, C. (2005). Apprendre à intervenir en formation initiale des enseignants. Communication orale présentée lors de la biennale de l'ARIS. Louvain-La-Neuve, 20 -22 Janvier 2005.

Ranganathan, M., \& Loquet, M. (2002). Approche historique de la danse indienne Kathak et choix des contenus d'enseignement en France : le lien entre sens et techniques. Communication affichée présentée lors de la biennale de l'ARIS. Rennes, 12 -14 Décembre 2002.

Redon, D. (2000). L'E.P.S. en section spécialisée de collège : Représentations sociales de la formation que devrait avoir l'intervenant en E.P.S. Communication affichée présentée lors de la biennale de l'ARIS. Grenoble, 14-16 Décembre 2000.

Redon, D. (2006). Pratique de sport adapté (déficience intellectuelle) : le cas du basketball. Communication affichée présentée lors de la biennale de l'ARIS. Besançon, 9-12 Mai 2006.

Refuggi, R., Louis, E., \& Deridder, M. (2005). Formation à l'intervention par l'appropriation de savoirs sur les techniques sportives. Une expérience en cours de réalisation. Communication orale présentée lors de la biennale de I'ARIS. Louvain-La-Neuve, 20 -22 Janvier 2005.

Rezig, M., Zghibi, M., \& Gréhaigne, JF. (2006). Analyse comparative de deux types d'enseignement des sports collectifs en Tunisie : pédagogie par objectifs et approche par compétences. Communication affichée présentée lors de la biennale de l'ARIS. Besançon, 9-12 Mai 2006.

Ria, L., \& Sève, C. ( 2002). Les engagements typiques des enseignants débutants en éducation physique et sportive. Communication orale présentée lors de la biennale de l'ARIS. Rennes, 12 -14 Décembre 2002.

Ria, L., \& Gal, N. (2002). La formation par l'action des enseignants débutants en éducation physique et sportive : la construction d'indices de typicalité, dans l'action en classe et par la réflexion dans l'action. Communication orale présentée lors de la biennale de l'ARIS. Rennes, 12 -14 Décembre 2002.

Ria, L., Bertone, S., Méard, JA., \& Euzet, JP. (2000). Les dilemmes des enseignants débutants en Éducation Physique. Communication affichée présentée lors de la biennale de l'ARIS. Grenoble, 14-16 Décembre 2000. 
Ridacker, D., \& Poggi, MP. (2006). Les références à l'origine des conceptions de l'enseignant d'EPS : le cas du volley-ball au collège. Communication affichée présentée lors de la biennale de l'ARIS. Besançon, 9-12 Mai 2006.

Rix, G. (2008). De l'appréhension de l'activité de l'arbitre en match à la question de son interaction avec les joueurs. Communication orale présentée lors de la biennale de l'ARIS. Rodez, 14-16 Mai 2008.

Rix, G., Biache, JM., \& Léziart, Y. (2000). Mettre en lumière des espaces vécus par une enseignante stagiaire EPS dans son inter-action avec les élèves. Communication affichée présentée lors de la biennale de l'ARIS. Grenoble, 14-16 Décembre 2000.

Robin, JF., \& Berthelot, C. (2002). Circonscription du phénomène de perte de figures au trampoline. Communication affichée présentée lors de la biennale de l'ARIS. Rennes, 12 -14 Décembre 2002.

Robin, JF., \& David, B. (2006). Étude des gestes professionnels d'enseignants dans trois disciplines : technologie, SVT, EPS. Communication orale présentée lors de la biennale de l'ARIS. Besançon, 9-12 Mai 2006.

Robin, JF., \& Lemonie, Y. (2008). Analyse des gestes professionnels des entraîneurs de l'équipe de France féminine de gymnastique. Communication orale présentée lors de la biennale de l'ARIS. Rodez, 14-16 Mai 2008.

Roesslé, S., \& Loquet, M. (2006). Construction précoce de dispositions à apprendre chez le jeune enfant : de l'activité aquatique familiale vers l'école. Communication orale présentée lors de la biennale de l'ARIS. Besançon, 9-12 Mai 2006.

Roesslé, S., \& Loquet, M. (2006). Construction précoce de dispositions à apprendre chez le jeune enfant : de l'activité aquatique familiale vers l'école. Communication orale présentée lors de la biennale de l'ARIS. Besançon, 9-12 Mai 2006.

Rolland, C., Cizeron, M., \& Biache, MJ. (2008). Les savoir-faire perceptifs experts des entraîneurs en gymnastique artistique. Communication orale présentée lors de la biennale de l'ARIS. Rodez, 14-16 Mai 2008.

Roncin, E., \& Loquet, M. (2006). Enseigner la danse à des adolescents avec autisme : : l'ingéniosité des gestes enseignants. Communication orale présentée lors de la biennale de l'ARIS. Besançon, 9-12 Mai 2006.

Rossard, C., Leveau, C., \& Testevuide, S. (2002). Evaluation du rapport de force au cours de l'action dans une situation d'apprentissage en badminton. Communication affichée présentée lors de la biennale de l'ARIS. Rennes, 12 -14 Décembre 2002. 
Roustan, C. (2005). L'aide à l'étude en moyenne section de maternelle : l'action d'une maîtresse polyvalente en EPS et biologie. Communication orale présentée lors de la biennale de I'ARIS. Louvain-La-Neuve, 20 -22 Janvier 2005.

Rouve, ME., \& Ria, L. (2008). Le développement professionnel d'enseignants d'EPS débutants en établissements labélisés " Ambition Réussite " : étude longitudinale de leurs interactions en classe et en dehors de la classe. Communication orale présentée lors de la biennale de l'ARIS. Rodez, 14-16 Mai 2008.

Roux, F., \& Durey, D. (2002). La production de connaissances technologiques pour la formation des entraîneurs de ski alpin. Communication orale présentée lors de la biennale de l'ARIS. Rennes, 12 -14 Décembre 2002.

Roux-Perez, T. (2005). Carrière des enseignants d'EPS et identité professionnelle : quels processus en jeu ? Communication orale présentée lors de la biennale de l'ARIS. Louvain-La-Neuve, 20 -22 Janvier 2005.

Roy, M., Beaudoin, C., Perreault, G., Turcotte, S., Spallanzani, C., \& Desbiens, JF. (2008). Formation à la communication pédagogique en entraînement sportif par la réflexion partagée s'inscrivant dans des démarches de supervision clinique et d'autosupervision. Communication orale présentée lors de la biennale de l'ARIS. Rodez, 14-16 Mai 2008.

Roy, M., Brunelle, JP., Perreault, G., Desbiens, JF., \& Spallanani, C. (2005). Processus d'implantation de l'entraînement à la prise de décision auprès d'un entraîneur de football américain universitaire. Communication orale présentée lors de la biennale de l'ARIS. Louvain-La-Neuve, 20 -22 Janvier 2005.

Roy, M., Desbiens, JF., Spallanzani, C., Lacasse, G., Lanoue, S., \& Brunelle, JP. (2005). Description du profil de communication d'entraîneurs en football américain : deux études de cas. Communication orale présentée lors de la biennale de l'ARIS. Louvain-La-Neuve, 20 -22 Janvier 2005.

Roy, M., Trudel, P., \& Lemyre, F. (2000). L'entrevue de type "récit d'expérience" afin de mieux comprendre l'expérience sportive. Communication affichée présentée lors de la biennale de l'ARIS. Grenoble, 14-16 Décembre 2000.

Saint Voirin, M., \& Gervais, F. (2000). Etude du savoir d'expérience d'un enseignant d'éducation physique au secondaire, perspective de " "l'action située ". Communication affichée présentée lors de la biennale de l'ARIS. Grenoble, 14-16 Décembre 2000.

Saintvoirin, M., Gal, N., \& Durand, M. (2005). Apprendre à faire une leçon d'Education physique : vers la construction de relations typiques entre actions et effets. 
Communication affichée présentée lors de la biennale de l'ARIS. Louvain-LaNeuve, 20 -22 Janvier 2005.

Saker, T., \& Cloes, M. (2008). Comparaison des objectifs attribués à l'éducation physique en Algérie et en Belgique francophone. Communication affichée présentée lors de la biennale de l'ARIS. Besançon, 9-12 Mai 2006.

Santiago, L., \& Ribeiro, AM. (2006). Représentation du corps des personnes âgées : une étude centrée dans leurs histoires de vie. Communication affichée présentée lors de la biennale de l'ARIS. Besançon, 9-12 Mai 2006.

Saujat, F., \& Laurent, Y. (2008). Controverses professionnelles et transmission du métier : le cas de professeurs conseillers pédagogiques en EPS. Communication orale présentée lors de la biennale de l'ARIS. Rodez, 14-16 Mai 2008.

Saury, J., \& Testevuide, S. (2002). L'activité collective comme articulation dynamique de cours d'action - L'exemple de la collaboration tactique au sein d'équipages experts en voile. Communication orale présentée lors de la biennale de l'ARIS. Rennes, 12 -14 Décembre 2002.

Sauvegrain, JP., Carnus, MF., Terrisse, A., \& Loizon, D. (2005). La prise en compte du sujet-élève dans une recherche sur l'appropriation du savoir par des élèves de cinquième, au cours d'un cycle de lutte. Communication orale présentée lors de la biennale de l'ARIS. Louvain-La-Neuve, 20 -22 Janvier 2005.

Schubauer-Leoni, M.L. (1996). Etude du contrat didactique pour des élèves en difficulté en mathématiques. Problématique didactique et/ou psychosociale. In C. Raisky, \& M. Caillot (Eds.) Au-delà des didactiques, le didactique. Bruxelles : De Boeck.

Serres, G. (2008). Analyse des interactions entre formateur universitaire, formateur de terrain et professeur stagiaire à l'échelle de l'année de formation. Communication orale présentée lors de la biennale de l'ARIS. Rodez, 14-16 Mai 2008.

Serres, G., Ria, L., Adé, D., \& Sève, C. (2005). Apprendre à intervenir en formation initiale des enseignants. Communication orale présentée lors de la biennale de I'ARIS. Louvain-La-Neuve, 20 -22 Janvier 2005.

Sicart, K., \& Maurice, JM. (2006). Les schèmes professionnels de l'éducateur sportif en citation : une co-construction enseignant-élève-cheval. Communication orale présentée lors de la biennale de l'ARIS. Besançon, 9-12 Mai 2006.

Siedentop, D. (1994). Sport education: Quality PE through positive sport experiences. Champaign, IL: Human Kinetics.

Silverman, S., \& Skonie, R. (1997). Research on teaching in physical education: an analysis of published research. JTPE, 16 (3), 300-311. 
Silverman, S., \& Manson, S. (2003). Research on teaching in physical education doctoral dissertations : a detailed investigation of focus, method, and analysis. JTPE, 22 (3), 280-297.

Spallanzani, C., Lemieux, GB., Dufresne, N., Desbiens, JF., Brunelle, JP., Goyette, R., Turcotte, S., \& Roy, M. (2006). Les didactiques au cœur du développement des compétences professionnelles dans un programme de formation en éducation physique. Communication orale présentée lors de la biennale de l'ARIS. Besançon, 9-12 Mai 2006.

Stoloff, S., Brunelle, JP., Roy, M., \& Spallanani, C. (2006). L'impact des occasions de répondre dans un processus d'intégration de l'entraînement à la prise de décision. Communication orale présentée lors de la biennale de l'ARIS. Besançon, 9-12 Mai 2006.

Terral, P., \& Collinet, C. (2008). Les savoirs scientifiques servent-ils à mieux intervenir ? Une étude sociologique des enseignants d'EPS et des entraîneurs au travail. Communication orale présentée lors de la biennale de l'ARIS. Rodez, 14-16 Mai 2008.

Tessier, D., \& Sarrazin, P. (2006). Effets d'un programme de formation au soutien de l'autonomie sur le style motivationnel d'enseignants d'EPS, la motivation et l'engagement des élèves. Communication orale présentée lors de la biennale de l'ARIS. Besançon, 9-12 Mai 2006.

Testevuide, S. (2000). Approche sémiotique de l'activité de l'orienteur. Communication affichée présentée lors de la biennale de l'ARIS. Grenoble, 14-16 Décembre 2000.

Thépaut, A., \& Léziart, Y. (2002). Le fonctionnement du contrat didactique en basket-ball à l'école élémentaire. Interventions du maître et avancée du savoir. Communication affichée présentée lors de la biennale de l'ARIS. Rennes, 12 -14 Décembre 2002.

Thépaut, A., \& Léziart, Y. (2005). Le fonctionnement du contrat didactique en basket-ball à l'école élémentaire. Techniques didactiques et avancée du savoir. Communication affichée présentée lors de la biennale de l'ARIS. Louvain-La-Neuve, 20 -22 Janvier 2005.

Thépaut, A. (2006). Le match de fin de séance : genre scolaire / genre didactique ? Communication affichée présentée lors de la biennale de l'ARIS. Besançon, 9-12 Mai 2006.

Theunissen, C., Langer, P., \& Cloes, M. (2008). Analyse de la formation des entraîneurs sportifs. Impact d'un cycle de cours de niveau 1. Communication affichée présentée lors de la biennale de l'ARIS. Rodez, 14-16 Mai 2008. 
Theunissen, C., Pauly, A., \& Cloes, M. (2006). Analyse du contexte de formation d'un jeune athlète. Etude de cas. Communication affichée présentée lors de la biennale de I'ARIS. Besançon, 9-12 Mai 2006.

Theunynck, D., Pezé, T., Bui-Xuan, G., \& Mikulovic, J. (2005). Pédagogie, conduites à tenir et cyberenseignement : de l'expérientiel à l'expertise. Etude préliminaire à propos d'une expérience de vulgarisation pédagogique par le cyberenseignement. Communication orale présentée lors de la biennale de l'ARIS. Louvain-La-Neuve, 20 -22 Janvier 2005.

Thomas-Bion, F., \& Fiard, J. (2008). Choix des pratiques sportives et conscience des risques encourus. Le poids de la loi ressenti par les enseignants d'EPS. Communication orale présentée lors de la biennale de l'ARIS. Rodez, 14-16 Mai 2008. Communication orale présentée lors de la biennale de l'ARIS. Rodez, 14-16 Mai 2008.

Thorel, S., \& David, B. (2006). Les effets du contexte didactique sur la coéducation en danse contemporaine au Collège. Communication orale présentée lors de la biennale de l'ARIS. Besançon, 9-12 Mai 2006.

Touboul, A., Carnus, MF., \& Terrisse, A. (2008). L'enseignant " chevronné " et l'enseignant " novice ". Analyse comparée des pratiques d'enseignement en éducation physique et sportive. Etude de cas contrastés en Savate Boxe Française. Communication orale présentée lors de la biennale de l'ARIS. Rodez, 14-16 Mai 2008.

Trépanier, A., Brunelle, JP., Stoloff, S., \& Desbiens, JF. (2006). Implanter l'entraînement à la prise de décision par une supervision clinique. Communication affichée présentée lors de la biennale de l'ARIS. Besançon, 9-12 Mai 2006.

Trohel, J., \& Crance, MC. (2008). L'activité d'un élève spécialiste de l'APSA enseignée ; nature et dynamique de ses préoccupations en cours d'EPS. Communication orale présentée lors de la biennale de l'ARIS. Rodez, 14-16 Mai 2008.

Trohel, J., \& Saury, J. (2006). Dynamique des interactions tuteur-stagiaire au cours des entretiens de conseil pédagogique. Communication orale présentée lors de la biennale de l'ARIS. Besançon, 9-12 Mai 2006.

Trohel, J. (2000). Interactions conseiller pédagogique - professeur stagiaire au cours d'entretiens de conseil post-leçons. Communication affichée présentée lors de la biennale de I'ARIS. Grenoble, 14-16 Décembre 2000. 
Trottin, B., \& Bazogue, N. (2002). Analyse des interventions d'un enseignant d'EPS : mise en évidence d'un fonctionnement stratégique et différencié. Communication affichée présentée lors de la biennale de l'ARIS. Rennes, 12 -14 Décembre 2002.

Trouilloud, D., \& Sarrazin, P. (2000). Les attentes des enseignants d'EPS sont-elles basées sur des indicateurs pertinents de la réussite finale des élèves ? Communication affichée présentée lors de la biennale de l'ARIS. Grenoble, 14-16 Décembre 2000.

Turcotte, S., Gaudreau, L., \& Otis, J. (2006). Les conceptions de l'enseignement en éducation à la santé d'éducateurs physiques du primaire. Communication affichée présentée lors de la biennale de l'ARIS. Besançon, 9-12 Mai 2006.

Turcotte, S., Roy, M., Desbiens, JF., Spallanzani, C., Goyette, R., Demers, J, \& Trudel, C. (2008). Les pratiques d'évaluation de l'enseignement en éducation à la santé d'éducateurs physiques du primaire. Communication affichée présentée lors de la biennale de l'ARIS. Rodez, 14-16 Mai 2008.

Ubaldi, JL., Berthier, S., \& Alin, C. (2006). CARGO : interdisciplinarité et formation des jeunes en alternance. Communication orale présentée lors de la biennale de l'ARIS. Besançon, 9-12 Mai 2006.

Uchan, K. (2002). Les contrats didactiques différentiels filles et garçons au collège : le cas de l'appui tendu renversé. Communication orale présentée lors de la biennale de l'ARIS. Rennes, 12 -14 Décembre 2002.

Uchan, K. (2002). Les contrats didactiques différentiels filles et garçons au collège : le cas de l'appui tendu renversé. Communication orale présentée lors de la biennale de l'ARIS. Rennes, 12 -14 Décembre 2002.

Uhlrich, G. (2005). Le cas particulier du projet de recherche " techno-didactique " du "Pocket Assistant Coach en rugby ". Communication orale présentée lors de la biennale de I'ARIS. Louvain-La-Neuve, 20 -22 Janvier 2005.

Ulhrich, G., Marty, Y., \& Bouthier, D. (2006).. Rôle des artefacts matériels et cognitifs dans le développement des compétences de l'intervenant éducatif en rugby. Communication orale présentée lors de la biennale de l'ARIS. Besançon, 9-12 Mai 2006

Vallée, B., Menaut, A., Lafont, L., \& Bouthier, D. (2006). Pratiques technicistes vs globales et évolution du niveau de clairvoyance de la norme d'internalité : une étude exploratoire sur le rapport au savoir. Communication orale présentée lors de la biennale de l'ARIS. Besançon, 9-12 Mai 2006. 
Vandercleyen, F., Carlier, G., \& Delens, C. (2008). Incidents critiques organisationnels vécus par des enseignants-stagiaires en éducation physique : du dilemme à l'autorégulation. Communication affichée présentée lors de la biennale de l'ARIS. Rodez, 14-16 Mai 2008.

Vandermeulen, M., \& Cloes, M. (2008). Analyse des structures valorisant le rôle social du sport en Communauté Wallonie-Bruxelles. Communication affichée présentée lors de la biennale de l'ARIS. Rodez, 14-16 Mai 2008.

Vandesande, I., Carlier, G., \& Dervaux, P. (2005). La danse à l'école : ce qu'en disent les enseignants. P. (2005). Communication orale présentée lors de la biennale de I'ARIS. Louvain-La-Neuve, 20 -22 Janvier 2005.

Vanlerberghe, G. (2008). Quel degré de liberté et autonomie accordé aux élèves ? Communication affichée présentée lors de la biennale de l'ARIS. Rodez, 14-16 Mai 2008.

Vanlerberghe, G., \& Bui-Xuan, G. (2005). Mesure de l'expertise de jeunes enseignants en EPS par l'association de mots. Communication orale présentée lors de la biennale de l'ARIS. Louvain-La-Neuve, 20 -22 Janvier 2005.

Vellet, J. (2005). Transmettre les qualités du geste dansé : une difficulté pour les professeurs de conservatoire. Communication orale présentée lors de la biennale de I'ARIS. Louvain-La-Neuve, 20 -22 Janvier 2005.

Vellet, J. (2006). Construire un "savoir danser" dans l'interaction maitre-élève. Communication orale présentée lors de la biennale de l'ARIS. Besançon, 9-12 Mai 2006.

Vellet, J. (2006). Construire un "savoir danser" dans l'interaction maitre-élève. Communication orale présentée lors de la biennale de I'ARIS. Besançon, 9-12 Mai 2006.

Vercruysse, B., André, E., \& Carlier, G. (2008). Contextualiser les apprentissages en volley-ball : à la recherche de l'expertise d'enseignants entraîneurs. Communication orale présentée lors de la biennale de l'ARIS. Rodez, 14-16 Mai 2008.

Vercruysse, B., Paquay, L., \& Carlier, G. (2008). Construire des contenus en sports collectifs : étude de la communication verbale et de la gestualité du professeur d'éducation physique. Communication orale présentée lors de la biennale de l'ARIS. Rodez, 14-16 Mai 2008.

Verscheure, I., \& Amade-Escot, C. (2005). Relations entre le genre et les représentations des élèves en volley-ball scolaire : difficultés d'objectivation dans une perspective 
d'intervention. Communication orale présentée lors de la biennale de I'ARIS. Louvain-La-Neuve, 20 -22 Janvier 2005.

Verscheure, I., \& Amade-Escot, C. (2005). Relations entre le genre et les représentations des élèves en volley-ball scolaire : difficultés d'objectivation dans une perspective. Communication orale présentée lors de la biennale de l'ARIS. Louvain-La-Neuve, 20 -22 Janvier 2005.

Viala, M., \& Lafont, L. (2005). Tutorat entre enfants et apprentissages moteurs Formation tutorielle : la différence entre des tuteurs non formés et des tuteurs formés. Communication orale présentée lors de la biennale de l'ARIS. Louvain-La-Neuve, 20 -22 Janvier 2005.

Vigneron, C. (2002). Filles et garçons en EPS au baccalauréat : quels contenus, quelles réussites ? Communication orale présentée lors de la biennale de l'ARIS. Rennes, 12 -14 Décembre 2002.

Vigneron, C. (2005). La reconnaissance chez les élèves des compétences professionnelles des enseignants d'EPS hommes et femmes. Communication orale présentée lors de la biennale de l'ARIS. Louvain-La-Neuve, 20 -22 Janvier 2005.

Vigneron, C. (2005). Les attentes spécifiques des lycéens relatives à l'EPS. Communication orale présentée lors de la biennale de l'ARIS. Louvain-La-Neuve, 20 -22 Janvier 2005.

Visioli, J., \& Ria, L ; (2008). Exploiter la dynamique émotionnelle du cours d'EPS : une activité des enseignants experts entre culture et singularités. Communication orale présentée lors de la biennale de l'ARIS. Rodez, 14-16 Mai 2008.

Voisard, N., Nachon, M., \& Mahut, N. (2002). Interprétation du risque en gymnastique dans l'enseignement primaire. Communication orale présentée lors de la biennale de l'ARIS. Rennes, 12 -14 Décembre 2002.

Vors, O., Gal-Petiffaux, N., \& Ciceron, M. (2008). L'EPS en classe 'Réseau ambition réussite' : un équilibre entre activités ostensives et clandestines. Communication orale présentée lors de la biennale de l'ARIS. Rodez, 14-16 Mai 2008.

Walczak, F., Brunelle, JP., Stoloff, S., \& Desbiens, JF. (2006). Selon des athlètes, quelles sont les caractéristiques d'un entraîneur efficace? Communication affichée présentée lors de la biennale de l'ARIS. Besançon, 9-12 Mai 2006.

Wallian, N., Poggi, MP., \& Musard, M. (2008). Co-construire des savoirs : les métiers de l'intervention dans les APSA. Besançon : PUFC. 
Wallian, N., Poggi, MP., \& Bonfils, S. (2008). Vers la construction d'une sémiotique de l'action collective : Productions graphiques et orales en classe de Hand Ball cycle 3. Communication orale présentée lors de la biennale de l'ARIS. Rodez, 14-16 Mai 2008.

Wane, C., Bouthier, D., \& David, B. (2006). Représentations sociales de la lutte sénégalaise avec frappe : perspectives d'élaboration de contenus. Communication affichée présentée lors de la biennale de l'ARIS. Besançon, 9-12 Mai 2006.

Wenger, E. (1998). Communities of practice. Cambridge, UK : Cambridge Press.

Zafour, M., \& Hamdi, M. (2005). La pratique de l'activité physique et sportive : attitudes et concepts chez les scolaires. Communication orale présentée lors de la biennale de l'ARIS. Louvain-La-Neuve, 20 -22 Janvier 2005.

Zerai, Z., \& Bedhioufi, H. (2008). Des outils numériques pour l'évaluation formative en sport collectif. Communication affichée présentée lors de la biennale de I'ARIS. Besançon, 9-12 Mai 2006. 